\title{
APPLICATION OF \\ THE GENERALIZED WEIERSTRASS PREPARATION THEOREM TO THE STUDY OF HOMOGENEOUS IDEALS
}

\author{
MUTSUMI AMASAKI
}

\begin{abstract}
The system of Weierstrass polynomials, defined originally for ideals in convergent power series rings, together with its sequence of degrees allows us to analyze a homogeneous ideal directly. Making use of it, we study local cohomology modules, syzygies, and then graded Buchsbaum rings. Our results give a formula which to some extent clarifies the connection among the matrices appearing in the free resolution starting from a system of Weierstrass polynomials, a rough classification of graded Buchsbaum rings in the general case and a complete classification of graded Buchsbaum integral domains of codimension two.
\end{abstract}

\section{INTRODUCTION}

In recent years computer scientists have been developing a constructive method of dealing with ideals in polynomial rings, among other things, the algorithm for finding a Gröbner basis of a given ideal and its syzygies (see [MM] for example). But so far, there seems to be few applications of their theory to the structural study of ideals as compared with the case of its counterpart, the generalized Weierstrass preparation theorem for ideals in formal and convergent power series rings originated by $\mathrm{H}$. Grauert and $\mathrm{H}$. Hironaka (cf. [Gr], $[\mathrm{Ga}],[\mathrm{H}],[\mathrm{HU}])$.

Irrespective of such movement in the algorithmic study of Gröbner bases, the present author began to use the system of Weierstrass polynomials (Gröbner basis with respect to generic coordinates satisfying some additional conditions) as the main tool for analyzing the homogeneous ideal defining a curve in $\mathbf{P}^{3}$ and obtained a lot of geometric and ring theoretic results (cf. [A1], [A2], [A3], [A4], [A5]). On the other hand, Gruson-Peskine in [GP] and Bolondi-Migliore in [BM1], [BM2] succeeded in proving many theorems concerning arithmetically Cohen-Macaulay or Buchsbaum curves in $\mathbf{P}^{3}$ with the use of what they call

Received by the editors October 14, 1988 and, in revised form, April 24, 1989. A summary of the first version of $\S \S 1-5$ was presented to the mini-semester "Commutative Algebra and Algebraic Geometry" held at Banach Center in Warsaw from April 5 to April 16 in 1988.

1980 Mathematics Subject Classification (1985 Revision). Primary 13A15, 13C05, 13D25, 13H10; Secondary 13D03, 14M05, $14 \mathrm{M} 07$.

Key words and phrases. System of Weierstrass polynomials, Gröbner basis, standard basis, local cohomology, free resolution, Buchsbaum ring. 
numerical character, which is in fact the same thing as the sequence of the degrees of Weierstrass polynomials of the ideal defining the generic hyperplane section of a curve (see (1.7)).

In view of these facts we will generalize and refine most of the results in [A1], $[A 2, \S \S 1,2]$ to give a perspective of such direct and constructive approach to the study of homogeneous ideals as mentioned above, and then illustrate how this method can be applied in higher dimensional and codimensional cases, taking graded Buchsbaum rings by way of example.

The first section is a preliminary part. After reviewing the generalized Weierstrass preparation theorem, we define a numerical invariant of a homogeneous ideal $I$ in a polynomial ring $R$, the basic sequence of $I$. This is the sequence of the degrees of Weierstrass polynomials of $I$ lined up by a definite rule (see $(1.5),[\mathrm{A} 2, \S 1])$. In $\S 2$, the relation between basic sequences and local cohomology modules is described briefly.

$\S \S 3$ and 4 are devoted to a detailed study of the free resolutions starting from systems of Weierstrass polynomials. Unlike the algorithm usually discussed by computer scientists, our method is based on a direct sum decomposition of an $R$-module $E$ of the form

$$
E=\bigoplus_{i=1}^{s} \bigoplus_{l=1}^{m_{i}} e_{l}^{i} k[x(i-1)]
$$

as a $k$-vector space (cf. Notation), so that we do not have to care about the ordering on monomials any more at this stage (see (3.3), [A1, Theorem 1.6]). Moreover successive application of our algorithm determines a free resolution for a module $E$ uniquely by the expression $(*)$, which we will call the standard free resolution and denote by $\Psi_{\bullet}: L_{\bullet} \rightarrow E$ (see (3.3), (3.4)). In the case $E=I$ (cf. (1.1.1)), though the standard free resolution $\Psi_{\bullet}: L_{\bullet} \rightarrow I$ starting from a system of Weierstrass polynomials is not necessarily minimal, it has some good properties. Its length is exactly the one determined by Auslander-Buchsbaum's theorem; a large part of the components of $\Psi_{p+1}$ can be obtained by rearranging those of $\Psi_{p}$ for $p \geq 1$; if $p \geq(r-c-1) / 2$ or $1 \leq p<(r-c-1) / 2$ and $\Psi_{p}$ satisfies a special condition, then $\Psi_{p+1}$ can be obtained by rearranging the components of $\Psi_{p}$, where $r=\operatorname{dim}(R), c=\operatorname{depth}_{\mathrm{m}}(R / I)$ (see (3.5), (3.11)). These properties are immediate consequences of our algorithm, but the proof of them other than the first requires hard computations. The readers may proceed from (3.4) directly to section five unless interested in (3.5), (3.11) or the proof of $(7.1)$.

Combining the results obtained in the first half with S.Goto's structure theorem for maximal Buchsbaum modules over regular local rings (cf. (5.1), [Go1], [Go2, (3.1)]), in the last three sections we study graded Buchsbaum rings with emphasis on characterization of the basic sequences of the ideals defining them. Our results are mostly generalizations of those proved before in [A2, $\S \S 3,4]$, $[A 5, \S 2]$ (see $(5.4)-(5.7),(5.12),(6.7),(7.4),(7.5))$. It should be noted that (5.4), (6.7) give a rough classification of graded Buchsbaum rings in terms of 
their basic sequences for all dimensions and codimensions. For the case of codimension two or dimension two (curves), there are other treatments in $\mathrm{M}$. Chang's recent work [C] (cf. (5.8), (7.1), (7.2)) and in [BM1], [BM2], [BM3], [BM4], [BBM], [EF], [GM1], [GM2] etc.

Notation. (i) The ground field $k$ is an infinite field of arbitrary characteristic unless otherwise specified.

(ii) Let $z_{1}, \ldots, z_{u}$ be indeterminates over a commutative ring $K$. We put $z(i)=\left(z_{i+1}, \ldots, z_{u}\right), K[z(i)]=K\left[z_{i+1}, \ldots, z_{u}\right]$, where $K[z(u)]=K$. Also under this condition we denote by $\operatorname{MAT}(z(i))$ the set of matrices with components in $K[z(i)]$.

(iii) For a matrix $\Upsilon=\left(v_{1}, \ldots, v_{q}\right)$ in a ring $K$ with columns $v_{1}, \ldots, v_{q}$ and a subring $K^{\prime}$ of $K$, we define $\operatorname{Im}^{K^{\prime}}(\Upsilon)=\left\{\sum_{l=1}^{q} a_{l} v_{l} \mid a_{l} \in K^{\prime}, 1 \leq l \leq q\right\}$.

(iv) The symbol $\oplus$ will be used in the following two senses: (1) $E^{\prime} \oplus E^{\prime \prime}=$ $\left\{\left(e^{\prime}, e^{\prime \prime}\right) \mid e^{\prime} \in E^{\prime}, e^{\prime \prime} \in E^{\prime \prime}\right\},(2) E^{\prime} \subset E, E^{\prime \prime} \subset E, E^{\prime} \cap E^{\prime \prime}=0, E^{\prime} \oplus E^{\prime \prime}=$ $\left\{e^{\prime}+e^{\prime \prime} \mid e^{\prime} \in E^{\prime}, e^{\prime \prime} \in E^{\prime \prime}\right\} \subset E$. The context will make it clear which it means.

(v) Given a graded module $D=\bigoplus_{t} D_{t}$, integers $p, q(q \geq 0)$ and a sequence of integers $w=\left(w_{1}, \ldots, w_{v}\right)$, we set $w+p=\left(w_{1}+p, \ldots, w_{v}+p\right)$, $(w+p)^{q}=(\underbrace{w+p, \ldots, w+p}_{q \text { times }})$ and $D(w)=\bigoplus_{l=1}^{v} D\left(w_{l}\right)$, where $D\left(w_{l}\right)_{t}=$ $D_{w_{l}+t}$ and the symbol $\oplus$ is used in the first sense.

(vi) Let $C=\bigoplus_{t \geq 0} C_{t}$ be a graded ring generated over $k=C_{0}$ by $C_{1}, \mathfrak{c}$ its irrelevant maximal ideal and $D$ a graded $C$-module. We put $\operatorname{dim}_{k}(D)_{t}=$ $\operatorname{dim}_{k}\left(D_{t}\right), h_{\mathrm{c}}^{j}(D)_{t}=\operatorname{dim}_{k}\left(H_{\mathrm{c}}^{j}(D)\right)_{t}, h_{\mathrm{c}}^{j}(D)=\sum_{t} h_{\mathrm{c}}^{j}(D)_{t}$. The length of $D$ and the multiplicity of $\mathfrak{c}$ with respect to $D$ will be denoted by $l_{C}(D)$ and $\operatorname{deg}(D)$ respectively. Note that $\operatorname{deg}(D)$ is the coefficient of the leading term of the polynomial $(\operatorname{dim}(D)) ! \sum_{l \leq t} \operatorname{dim}_{k}(D)_{l}$ in $t>>0, \operatorname{dim}(D)$ denoting the Krull dimension of $D$.

(vii) We denote the $p \times p$ identity matrix by $1_{p}$. (viii)

$$
\left(\begin{array}{l}
v \\
u
\end{array}\right)_{+}=\frac{v !}{(v-u) ! u !} \text { if } v \geq u \geq 0 \text { and }\left(\begin{array}{l}
v \\
u
\end{array}\right)_{+}=0 \quad \text { otherwise. }
$$

We have $\left(\begin{array}{c}n+1 \\ u\end{array}\right)_{+}-\left(\begin{array}{l}v \\ u\end{array}\right)_{+}=\left(\begin{array}{c}v \\ u-1\end{array}\right)_{+}$for all integers $v$ and $u \geq 1$.

(ix) $\mathbf{Z}_{0}=\{t \mid t \in \mathbf{Z}, t \geq 0\}$.

\section{Systems of Weierstrass POlynomials}

Let us begin by reviewing the generalized Weierstrass preparation theorem in the case of homogeneous ideals (cf. [Gr], [Ga], [H], [HU]). We will use the linear order $\prec$ on $\mathbf{Z}_{0}^{r}(r \geq 1)$ which is defined as follows (cf. [Gr, p. 179]): $\left(\mu_{1}, \ldots, \mu_{r}\right) \prec\left(\nu_{1}, \ldots, \nu_{r}\right)$ if and only if there is an integer $i(1 \leq i \leq r)$ such that $\mu_{i}<\nu_{i}$ and $\mu_{j}=\nu_{j}$ for $i<j \leq r$. This order is equivalent to the reverse lexicographic order (cf. [BS, (2.1)]). 
Let $R$ be a polynomial ring over $k$ generated by $r$ indeterminates $y_{1}, \ldots, y_{r}$ and $m$ its maximal ideal $\left(y_{1}, \ldots, y_{r}\right) R$. We call an element of $R_{1}:=\sum_{i=1}^{r} k y_{i}$ a linear form of $R$. The vector space $R_{1}^{\oplus t}$ will be regarded as an $r t$-dimensional affine space in a natural way for $t \geq 1$. Let $x=\left(x_{1}, \ldots, x_{r}\right)$ be an $r$-tuple of linear forms of $R$ such that $R=k\left[x_{1}, \ldots, x_{r}\right]$. Given a nonzero homogeneous polynomial $f=\sum_{|\nu|=n} a_{\nu} x^{\nu}(n=\operatorname{deg}(f))$ in $R$, we denote by in $(x ; f)$ the monomial $a_{\mu} x^{\mu}$ occurring in $f$, whose multiexponent $\mu$ is the minimum of $\left\{\nu \mid a_{\nu} \neq 0\right\}$ with respect to the order $\prec$. Furthermore for a homogeneous ideal $I$ in $R$ we define in $(x ; I)$ to be the ideal in $R$ generated by $\{\operatorname{in}(x ; f) \mid f \in$ $I \backslash\{0\}\}$.

Although the arguments in [HU], [U] are carried out with the usual lexicographic order, the results in them are all valid with respect to the order $\prec$ as well as the similar results in [Gr], [Ga].

Theorem (1.1) (H. Grauert, H. Hironaka). Let I be a nontrivial homogeneous ideal in $R$. Then there are integers $c, m_{i}\left(0 \leq c<r, 1 \leq i \leq r-c, m_{i} \geq 1\right)$, nondecreasing sequences of positive integers $n^{i}=\left(n_{1}^{i}, \ldots, n_{m_{i}}^{i}\right)(1 \leq i \leq r-$ c) and a nonempty Zariski open subset $U$ of $R_{1}^{\oplus r}$ such that for every $x=$ $\left(x_{1}, \ldots, x_{r}\right) \in U$, one can find a system of generators $\left\{f_{l}^{i} \mid 1 \leq i \leq r-c, 1 \leq l \leq\right.$ $\left.m_{i}\right\}$ of $I$ having the following properties with respect to $x$.

(1) Each $f_{l}^{i}\left(1 \leq i \leq r-c, 1 \leq l \leq m_{i}\right)$ is a homogeneous polynomial of degree $n_{l}^{i}$ different from zero and

$$
I=\bigoplus_{i=1}^{r-c} \bigoplus_{l=1}^{m_{i}} f_{l}^{i} k[x(i-1)]
$$

(2) The monomial in $\left(x ; f_{l}^{i}\right)$ belongs to $k\left[x_{1}, \ldots, x_{i}\right] x_{i}$ for all $i, l$ and

$$
\operatorname{in}(x ; I)=\bigoplus_{i=1}^{r-c} \bigoplus_{l=1}^{m_{i}} \operatorname{in}\left(x ; f_{l}^{i}\right) k[x(i-1)] .
$$

(3) For each $p(1 \leq p \leq r-c)$, let $N(x ; I, p)$ denote the linear subspace of $R$ spanned over $k$ by all the monomials not contained in any

$$
\operatorname{in}\left(x ; f_{l}^{i}\right) k[x(i-1)] \quad\left(1 \leq i \leq p, 1 \leq l \leq m_{i}\right)
$$

Then

$$
R=\left\{\bigoplus_{i=1}^{p} \bigoplus_{l=1}^{m_{i}} f_{l}^{i} k[x(i-1)]\right\} \oplus N(x ; I, p)
$$

and moreover $f_{l}^{i}-\operatorname{in}\left(x ; f_{l}^{i}\right) \in N(x ; I, r-c)$ for all $i, l(1 \leq i \leq r-c, 1 \leq l \leq$ $\left.m_{i}\right)$.

Furthermore the open set $U$ can be chosen so that the monomial ideal in $(x ; I)$ satisfies the following condition for every $x \in U$. 
(4) [U, Theorem 2.2] If $x_{1}^{\mu_{1}} \cdots x_{p}^{\mu_{p}} \cdots x_{q}^{\mu_{q}} \cdots x_{r}^{\mu_{r}} \in \operatorname{in}(x ; I)$ with $1 \leq p<$ $q \leq r, \mu_{q}>0$, then $x_{1}^{\mu_{1}} \cdots x_{p}^{\mu_{p}+t} \cdots x_{q}^{\mu_{q}-t} \cdots x_{r}^{\mu_{r}} \in \operatorname{in}(x ; I)$ for all $t$ such that

$$
\left(\begin{array}{c}
\mu_{q} \\
t
\end{array}\right) \not \equiv 0 \quad(\bmod \operatorname{char}(k)), \quad 0 \leq t \leq \mu_{q} \text {. }
$$

That is in $(x ; I)$ is Borel fuxed (cf. [BS, (2.6)]).

Proof. The readers can find the proof for the case $I$ is an ideal in a convergent power series ring over $\mathbf{C}$ in [Gr, §2], [HU, §5], [Ga]. For 4), see [U]. A sketch of the proof based on [HU] is given in Appendix for those who are not familiar with these works.

Remark (1.2). (1) The system of generators described in the above theorem is called a system of Weierstrass polynomials (cf. [Gr, §2, Definition 1]). Also we often call it a standard basis or a Gröbner basis.

(2) The condition (2) of (1.1) implies that $f_{l}^{i}\left(x_{1}, \ldots, x_{q}, 0, \ldots, 0\right)=0$ for all $i, l, q\left(0 \leq q<i \leq r-c, 1 \leq l \leq m_{i}\right)$. Given an integer $p(0 \leq p \leq r-1)$, we put $\bar{c}=\max (0, c-p)$. Then the set $\left\{f_{l}^{i}\left(x_{1}, \ldots, x_{r-p}, 0, \ldots, 0\right) \mid 1 \leq i \leq\right.$ $\left.r-p-\bar{c}, 1 \leq l \leq m_{i}\right\}$ is the system of generators of the homogeneous ideal $\bar{I}:=I+x(r-p) R / x(r-p) R$ in $\bar{R}:=R / x(r-p) R$ satisfying the conditions (1), (2), (3) of (1.1) with respect to the variables $x_{1}, \ldots, x_{r-p}$.

Lemma (1.3). Notation being as in (1.1), we have

(1) for each $p(1 \leq p \leq r-c)$ the vector space $N(x ; I, p)$ is a free $k[x(p)]$ module,

(2) $c=\operatorname{depth}_{\mathrm{m}}(R / I)$, in particular $0 \leq c \leq \operatorname{dim}(R / I)$.

Proof. (1) Write each monomial of $R$ in the form $a x^{\mu} x^{\nu}$ with $a \in k$, $\mu=\left(\mu_{1}, \ldots, \mu_{p}, 0, \ldots, 0\right), \nu=\left(0, \ldots, 0, \nu_{p+1}, \ldots, \nu_{r}\right) \in \mathbf{Z}_{0}^{r}$. Then $a x^{\mu} x^{\nu} \in$ $N(x ; I, p)$ if and only if $x^{\mu} \in N(x ; I, p)$ or $a=0$ by the definition and (2) of (1.1). Hence the monomials of $N(x ; I, p) \cap k\left[x_{1}, \ldots, x_{p}\right]$ form a free basis of $N(x ; I, p)$ over $k[x(p)]$.

(2) Put $A=R / I, c^{\prime}=\operatorname{depth}_{\mathrm{m}}(A)$. We may assume with no loss of generality that $x_{r-c^{\prime}+1}, \ldots, x_{r}$ is an $A$-regular sequence. Notice first that by (3) of (1.1) and (2) of (1.2) the natural map $\xi_{p}: N(x ; I, r-p) \rightarrow A \quad(c \leq p \leq r-$ $1)$ is a surjective homomorphism of $k[x(r-p)]$-modules, which becomes an isomorphism when considered modulo $x(r-p) k[x(r-p)]$. Since $\xi_{c}$ is an isomorphism, we have $c \leq c^{\prime}$ by (1). On the other hand, since $x_{r-c^{\prime}-1}, \ldots, x_{r}$ is $A$-regular, we find $\operatorname{Tor}_{1}^{k\left[r-c^{\prime}\right]}(k, A)=0, \operatorname{Ker}\left(\xi_{c^{\prime}}\right) \otimes_{k\left[x\left(r-c^{\prime}\right)\right]} k=0$ and therefore $\operatorname{Ker}\left(\xi_{c^{\prime}}\right)=0$. In other words $I \cap N\left(x ; I, r-c^{\prime}\right)=0$. If $c<c^{\prime}$, then $f_{1}^{r-c}$ would be an element of $I \cap N\left(x ; I, r-c^{\prime}\right)$ different from zero by (1), (2), (3) of (1.1), which is a contradiction. Hence $c=c^{\prime}$.

Let $I$ be a nontrivial homogeneous ideal in $R$. With the notation of the above theorem the direct sum decomposition (1.1.1) applied to $I+$ 
$x(r-p) R / x(r-p) R$ yields

$$
\operatorname{dim}_{k}(I+x(r-p) R / x(r-p) R)_{t}=\sum_{i=1}^{r-p-\bar{c}} \sum_{l=1}^{m_{i}}\left(\begin{array}{c}
t-n_{l}^{i}+r-p-i \\
r-p-i
\end{array}\right)_{+}
$$

for all $x \in U, t \in \mathbf{Z}$ and $p(0 \leq p \leq r-1)$, where $\bar{c}=\max (0, c-p)$ (see (2) of (1.2)). This formula determines the sequences $n^{1}, \ldots, n^{r-c}$ uniquely one by one along with $c, m_{i}(1 \leq i \leq r-c)$. These numerical data therefore depend only on the ideal itself and can be viewed as an invariant for $I$.

Definition (1.5). For a nontrivial homogeneous ideal $I$ in $R$, let $n^{i}=\left(n_{1}^{i}, \ldots\right.$, $\left.n_{m_{i}}^{i}\right)(1 \leq i \leq r-c)$ be the nondecreasing sequences of integers stated in (1.1). We call the sequence $\left(n^{i}\right)_{1 \leq i \leq r-c}=\left(n^{1} ; \ldots ; n^{i} ; \ldots ; n^{r-c}\right)$ the basic sequence of $I$ and denote it by $B(I)$.

Proposition (1.6). Let I be a nontrivial homogeneous ideal in $R$ and

$$
\left(n^{i}\right)_{1 \leq i \leq r-c}, \quad n^{i}=\left(n_{1}^{i}, \ldots, n_{m_{i}}^{i}\right)
$$

its basic sequence. Put $d=\operatorname{dim}(R / I)$.

(1) We have $m_{1}=1$,

$$
m_{j}=(-1)^{j-1} \sum_{i=1}^{j-1}(-1)^{i} \sum_{l=1}^{m_{i}}\left(\begin{array}{c}
n_{l}^{i} \\
j-i
\end{array}\right)
$$

for $2 \leq j \leq r-d$ and

$$
\operatorname{deg}(R / I)=(-1)^{r-d} \sum_{i=1}^{r-d}(-1)^{i} \sum_{l=1}^{m_{i}}\left(\begin{array}{c}
n_{l}^{i} \\
r-d+1-i
\end{array}\right)-m_{r-d+1},
$$

where $m_{r-d+1}$ is understood to be zero in the case $d=c$.

(2) $n_{1}^{i} \leq n_{1}^{i+1}$ for $1 \leq i \leq r-c-1$.

Proof. (1) Put $A=R / I$. Applying (1.4) with $p=0$, we get

$$
l_{R}\left(R / I+\mathrm{m}^{t+1}\right)=\left(\begin{array}{c}
t+r \\
r
\end{array}\right)-\sum_{i=1}^{r-c} \sum_{l=1}^{m_{i}}\left(\begin{array}{c}
t-n_{l}^{i}+r-i+1 \\
r-i+1
\end{array}\right)
$$

for $t>>0$. Denote the right-hand side of this equality by $P(t)$. Since $P(t)$ is a polynomial in $t$ of degree $d$ with leading term $(\operatorname{deg}(A) / d !) t^{d}$, the $p$-difference

$$
\Delta^{p} P(t)=\left(\begin{array}{c}
t+r-p \\
r-p
\end{array}\right)-\sum_{i=1}^{q} \sum_{l=1}^{m_{i}}\left(\begin{array}{c}
t-n_{l}^{i}+r-i-p+1 \\
r-i-p+1
\end{array}\right)
$$

$(q=\min (r-c, r-p+1))$ is a polynomial in $t$ of degree $d-p$ with leading term $(\operatorname{deg}(A) /(d-p) !) t^{d-p}$ for $0 \leq p \leq d$ but vanishes identically for $p>d$. Clearly $m_{1}=1$. For each $j(2 \leq j \leq r-d)$, it follows from the above observation that

$$
\Delta^{r-j+1} P(t)=\left(\begin{array}{c}
t+j-1 \\
j-1
\end{array}\right)-\sum_{i=1}^{j} \sum_{l=1}^{m_{i}}\left(\begin{array}{c}
t-n_{l}^{i}+j-i \\
j-i
\end{array}\right)=0
$$


for all integers $t$. We find therefore by putting $t=-1$ that

$$
-\sum_{i=1}^{j-1} \sum_{l=1}^{m_{i}}(-1)^{j-i}\left(\begin{array}{c}
n_{l}^{i} \\
j-i
\end{array}\right)-m_{j}=0 .
$$

This proves the first formula. To show the second, substitute -1 for $t$ in the identity $\Delta^{d} P(t)=\operatorname{deg}(A)$.

(2) Let $\left\{f_{l}^{i} \mid 1 \leq i \leq r-c, 1 \leq l \leq m_{i}\right\}$ be the system of generators of $I$ having the properties described in (1.1) with respect to a generic $x=\left(x_{1}, \ldots, x_{r}\right)$. Write $\operatorname{in}\left(x ; f_{1}^{i+1}\right)=a_{i+1} x_{1}^{\mu_{1}} \cdots x_{i}^{\mu_{i}} x_{i+1}^{\mu_{i+1}}$ with $a_{i+1} \in k \backslash\{0\}, \mu_{i+1}>0$ for $1 \leq i \leq r-c-1$. It follows from (2) and (4) of (1.1) that $x_{1}^{\mu_{1}} \cdots x_{i}^{\mu_{i}} \notin \operatorname{in}(x ; I)$ but $g:=x_{1}^{\mu_{1}} \cdots x_{i}^{\mu_{i}+\mu_{i+1}} \in \operatorname{in}(x ; I)$, therefore there is an $f_{l}^{i}\left(1 \leq l \leq m_{i}\right)$ such that $g=\operatorname{in}\left(x ; f_{l}^{i}\right) b x_{i}^{t}$ with some $b \in k \backslash\{0\}, t \geq 0$. Hence $n_{1}^{i} \leq n_{l}^{i}=\operatorname{deg}\left(f_{l}^{i}\right) \leq$ $\operatorname{deg}\left(f_{1}^{i+1}\right)=n_{1}^{i+1}$.

Remark (1.7). (1) Note that $n_{1}^{1}=\min \left\{t \mid I_{t} \neq 0\right\}, m_{2}=n_{1}^{1}$.

(2) Suppose $r=4, \operatorname{dim}(R / I)=2, \operatorname{depth}_{\mathfrak{m}}(R / I) \geq 1$. In this case the basic sequence of $I$ defined in this section coincides with that defined in [A2, $\S 1]$. Moreover, let $I^{\prime}$ be the ideal in $R^{\prime}:=k\left[y_{1}, y_{2}, y_{3}\right]$ determined by the condition $R^{\prime} / I^{\prime}=(R / I+(h)) / H_{\mathrm{m}}^{0}(R / I+(h))$ for a generic linear form $h \in R_{1}$ and let $\left(n_{1}^{1} ; n_{1}^{2}, \ldots, n_{v}^{2}\right) \quad\left(v=n_{1}^{1}\right)$ be the basic sequence of $I^{\prime}$. Then the numerical character of $\operatorname{Proj}(R / I)$ defined and used in [GP], [BM1], [BM2] is nothing but the nonincreasing sequence $\left(n_{v}^{2}, \ldots, n_{1}^{2}\right)$.

\section{BASIC SEQUENCES AND LOCAL COHOMOLOGY}

The basic sequence of a homogeneous ideal provides a lot of information on the local cohomology modules of the factor ring associated with it. The purpose of this section is to give a brief description of this fact. We fix a nontrivial homogeneous ideal $I$ in $R$ and a generic $r$-tuple $x=\left(x_{1}, \ldots, x_{r}\right)$ of linear forms of $R$. Set $A=R / I, c=\operatorname{depth}_{\mathrm{m}}(A), d=\operatorname{dim}(A)$. Let $\left(n^{i}\right)_{1 \leq i \leq r-c}, n^{i}=$ $\left(n_{1}^{i}, \ldots, n_{m_{i}}^{i}\right)$ be the basic sequence of $I$ (cf. (1.3)).

Lemma (2.1). If $c=0$, there are positive integers $p_{l}\left(1 \leq l \leq m_{r}\right)$ such that

$$
H_{\mathfrak{m}}^{0}(A) \cong \bigoplus_{l=1}^{m_{r}}\left(k\left[x_{r}\right] /\left(x_{r}^{p_{l}}\right)\right)\left(-n_{l}^{r}+p_{l}\right)
$$

as a $k\left[x_{r}\right]$-module.

Proof. Let the notation be as in (1.1). We have $n_{l}^{i}=\operatorname{deg}\left(f_{l}^{i}\right)$ for all $i, l$. There are distinct monomials $g_{l}^{\prime} \in k\left[x_{1}, \ldots, x_{r-1}\right]$ and positive integers $p_{l}(1 \leq l \leq$ $\left.m_{r}\right)$ such that $\operatorname{in}\left(x ; f_{l}^{r}\right)=g_{l}^{\prime} x_{r}^{p_{l}}$ by (2) of (1.1). Furthermore by the definition of the order $\prec$ there is a homogeneous polynomial $g_{l} \in R$ such that $f_{l}^{r}=g_{l} x_{r}^{p_{l}}$, in $\left(x ; g_{l}\right)=g_{l}^{\prime}$ for each $l\left(1 \leq l \leq m_{r}\right)$. Let $C$ and $D^{\prime}$ denote the subspaces of $N:=N(x ; I, r)$ generated over $k$ by the sets of monomials 
$\left\{x^{\nu} \mid x^{\nu} x_{r}^{t} \in N\right.$ for all $\left.t \geq 0\right\}$ and $\left\{x^{\nu} \mid x^{\nu} \in N, x^{\nu} x_{r}^{t} \notin N\right.$ for some $\left.t \geq 1\right\}$ respectively. If $x^{\nu} \in D^{\prime}$, then $x^{\nu} x_{r}^{t} \in \bigoplus_{l=1}^{m_{r}}$ in $\left(x ; f_{l}^{r}\right) k\left[x_{r}\right]$ by (2) of (1.1), so that $x^{\nu}=g_{l}^{\prime} x_{r}^{u}$ for some $l, u\left(1 \leq l \leq m_{r}, 0 \leq u<p_{l}\right)$. On the other hand, monomials of this form must lie in $D^{\prime}$. Consequently $D^{\prime}=\bigoplus_{l=1}^{m_{r}} \bigoplus_{u=0}^{p_{l}-1} k g_{l}^{\prime} x_{r}^{u}, N=$ $C \oplus D^{\prime}$. Set $D=\sum_{l=1}^{m_{r}} \sum_{u=0}^{p_{l}-1} k g_{l} x_{r}^{u} \subset R$. This sum is indeed a direct sum, since in $\left(x ; g_{l}\right) \neq \operatorname{in}\left(x ; g_{l^{\prime}}\right)$ for $l \neq l^{\prime}$. Note that in $(x ; h) \in D^{\prime}$ for $h \in D$ different from zero. We first prove the following

Claim (2.1.1). $R=I \oplus C \oplus D$.

Suppose $f+g+h=0$ with $f \in I, g \in C, h \in D$. Then $g x_{r}^{t} \in C \cap I=0$ for $t$ large enough, so that $g=0$ and it follows from the equalities $f=$ $-h$, in $(x ; I) \cap D^{\prime}=0$ that $f=h=0$. The sum $I+C+D$ is therefore a direct sum of $k$-vector spaces. Since $\operatorname{dim}_{k}\left(D^{\prime}\right)=\operatorname{dim}_{k}(D)$ and $R /(I \oplus C) \cong D^{\prime}$ by (3) of (1.1), we find $R=I \oplus C \oplus D$.

It is now easy to prove our lemma. Let $H$ denote the graded $k\left[x_{r}\right]$-module $\bigoplus_{l=1}^{m_{r}}\left(k\left[x_{r}\right] /\left(x_{r}^{p_{l}}\right)\right)\left(-n_{l}^{r}+p_{l}\right)$. We associate with each $\left(g, \bar{h}_{1}, \ldots, \bar{h}_{r}\right) \in C \oplus H$ the element of $A$ represented by $g+\sum_{l=1}^{m_{r}} g_{l} h_{l}$, where $h_{l}$ is a polynomial of $k\left[x_{r}\right]$ which represents $\bar{h}_{l}\left(1 \leq l \leq m_{r}\right)$. Since $g_{l} x_{r}^{p_{l}} \in I$ for all $l\left(1 \leq l \leq m_{r}\right)$, this yields a well-defined homomorphism $\xi: C \oplus H \rightarrow A$ of graded $k\left[x_{r}\right]$-modules of degree zero, which is in fact an isomorphism by (2.1.1). We may assume that the variable $x_{r}$ is chosen sufficiently generally so that it is a member of a system of parameters for $A$ in the case $\operatorname{dim}(A) \geq 1$, therefore in any case $H_{\mathrm{m}}^{0}(A)=0:_{A} x_{r}^{t}$ for all large enough $t$. Hence $H_{\mathrm{m}}^{0}(A) \cong H$. Q.E.D.

The short exact sequences

$$
\begin{gathered}
0 \rightarrow\left(0:_{A} x_{r}\right)(-1) \rightarrow A(-1) \stackrel{x_{r}}{\longrightarrow} x_{r} A \rightarrow 0, \\
0 \rightarrow x_{r} A \rightarrow A \rightarrow A / x_{r} A \rightarrow 0
\end{gathered}
$$

give rise to a long exact sequence

$$
\begin{aligned}
H_{\mathrm{m}}^{j-1}(A)(-1) & \stackrel{x_{r}}{\longrightarrow} H_{\mathrm{m}}^{j-1}(A) \\
& \rightarrow H_{\mathrm{m}}^{j-1}\left(A / x_{r} A\right) \rightarrow H_{\mathrm{m}}^{j}(A)(-1) \stackrel{x_{r}}{\rightarrow} H_{\mathrm{m}}^{j}(A) \quad(j \geq 1),
\end{aligned}
$$

since $0:_{A} x_{r}$ has finite length by the choice of $x_{1}, \ldots, x_{r}$.

Corollary (2.3). We have the following formulae:

(1) $\max \left\{t \mid H_{\mathrm{m}}^{0}(A / x(r-p) A)_{t} \neq 0\right\}=n_{m_{r-p}}^{r-p}-1$ for all $p(c \leq p<r)$,

(2) $H_{\mathrm{m}}^{j}(A)_{t}=0$ for all $j, t$ such that $c \leq j<r, t \geq n_{m_{r-j}}^{r-j}-j$,

(3) if $j=c$ or $c<j<r$ and $n_{m_{r-j+1}}^{r-j+1}<n_{m_{r-j}}^{r-j}$, then

$$
H_{\mathrm{m}}^{j}(A)_{t} \neq 0
$$

for $t=n_{m_{r-j}}^{r-j}-j-1$. 
Proof. (1) The basic sequence of the ideal $I+x(r-p) R / x(r-p) R$ in $R / x(r-p) R$ is $\left(n^{i}\right)_{1 \leq i \leq r-p}$ for $c \leq p<r$ by (2) of (1.2), so that the formula follows from $(2 . \overline{1})$.

(2) If $r=1$, then $c=j=0$ and $H_{\mathrm{m}}^{0}(A)_{t}=0$ for all $t \geq n_{m_{1}}^{1}$ by (2.1). Suppose $r>1$ and the formula holds for smaller values of $r$. Put $\bar{c}=\max (0, c-1)$. Since $\left(n^{i}\right)_{1 \leq i \leq r-1-\bar{c}}$ is the basic sequence of $\bar{I}:=I+$ $x_{r} R / x_{r} R \subset R / x_{r} R=k\left[x_{1}, \ldots, x_{r-1}\right]$, we have $H_{\mathrm{m}}^{j}\left(A / x_{r} A\right)_{t}=0$ for all $j, t$ such that $\bar{c} \leq j<r-1, t \geq n_{m_{r-1-j}}^{r-1-j}-j$ by the induction hypothesis. It then follows from the sequence (2.2) that $H_{\mathrm{m}}^{j}(A)_{t} \stackrel{x_{r}}{\longrightarrow} H_{\mathrm{m}}^{j}(A)_{t+1}$ is an injection for every $j, t$ satisfying $\bar{c}+1 \leq j<r, t \geq n_{m_{r-j}}^{r-j}-j$. Since $H_{\mathrm{m}}^{j}(A)_{t}=0$ for all sufficiently large $t$, we conclude that the desired formula holds except for the case $c=j=0, t \geq n_{m_{r}}^{r}$. But this case is obvious by (1).

(3) With the same notation as above, we proceed again by indution on $r$. When $j=c=0$, in particular when $r=1$, we are done by (1). Suppose $r>1$ and the assertion holds for smaller values of $r$. Put $t=n_{m_{r-j}}^{r-j}-j-1$. It is enough to consider the case $j>0$. By hypothesis we have $j-1=\bar{c}$ or

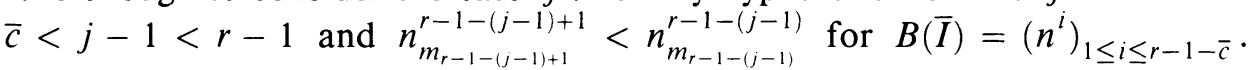
It follows therefore from the induction hypothesis that $H_{\mathrm{m}}^{j-1}\left(A / x_{r} A\right)_{t+1} \neq 0$. On the other hand $H_{\mathrm{m}}^{j-1}(A)_{t+1}=H_{\mathrm{m}}^{j}(A)_{t+1}=0$ by (2) or the equality $c=$ $\operatorname{depth}_{\mathrm{m}}(A)$, so that $H_{\mathrm{m}}^{j}(A)_{t} \neq 0$ by $(2.2)$.

Since $H_{\mathrm{m}}^{j}(A)=0$ for all $j>d$, the formula (3) above implies the following Corollary (2.4). $n_{m_{i}}^{i} \leq n_{m_{i+1}}^{i+1}$ for $1 \leq i \leq r-d-1$.

Proposition (2.5) (cf. [BS, (2.4)]). The regularities of $I$ and $A$ are given by the following formula:

$$
\begin{aligned}
\max & \left\{t \mid H_{\mathrm{m}}^{j}(I)_{t-j} \neq 0 \text { for some } j \geq 0\right\} \\
& =\max \left\{t \mid H_{\mathrm{m}}^{j}(A)_{t-j} \neq 0 \text { for some } j \geq 0\right\}+1 \\
& =\max \left\{n_{l}^{i} \mid 1 \leq i \leq r-c, 1 \leq l \leq m_{i}\right\} .
\end{aligned}
$$

Proof. We will first prove the second equality. Put

$$
t_{0}=\max \left\{n_{l}^{i} \mid 1 \leq i \leq r-c, 1 \leq l \leq m_{i}\right\} .
$$

Obviously $H_{\mathrm{m}}^{j}(A)_{t-j}=0$ for all $j \geq 0, t \geq t_{0}$ by (2) of (2.3). Let $j(c \leq j<r)$ be the integer such that $n_{m_{r-j}}^{r-j}=t_{0}$ and $n_{m_{r-j^{\prime}}}^{r-j^{\prime}}<t_{0}$ for all $j^{\prime}\left(c \leq j^{\prime}<j\right)$. Then $j=c$ or $c<j<r$ and $n_{m_{r-j+1}-j+1}^{r-j} n_{m_{r-j}}^{r-j}$, so that $H_{\mathfrak{m}}^{j}(A)_{t_{0}-j-1} \neq 0$ by (3) of (2.3). Hence

$$
t_{0}-1=\max \left\{t \mid H_{\mathrm{m}}^{j}(A)_{t-j} \neq 0 \text { for some } j \geq 0\right\} .
$$


The first equality follows from the isomorphisms $H_{\mathrm{m}}^{j}(A) \cong H_{\mathrm{m}}^{j+1}(I)(0 \leq j \leq$ $r-2)$ and the exact sequence $0 \rightarrow H_{\mathfrak{m}}^{r-1}(A) \rightarrow H_{\mathfrak{m}}^{r}(I) \rightarrow H_{\mathfrak{m}}^{r}(R) \rightarrow 0$, since $t_{0}>0$.

Proposition (2.6) (cf. [EG]). The homogeneous ideal I has a p-linear free resolution if and only if $n_{l}^{i}=p$ for all $i, l\left(1 \leq i \leq r-c, 1 \leq l \leq m_{i}\right)$.

Proof. If $I$ has a $p$-linear free resolution, then $n_{1}^{1}=p$ and $H_{\mathrm{m}}^{j}(I)_{t-j}=0$ for all $j \geq 0, t \geq p+1$. Therefore $n_{l}^{i} \leq p$ for all $i, l\left(1 \leq i \leq r-c, 1 \leq l \leq m_{i}\right)$ by (2.5), which together with (2) of (1.6) implies the necessity of the condition. Conversely if this condition is satisfied by $B(I)$, then $I$ has a $p$-linear free resolution by (2.5) and [EG, (1) of 1.2].

Remark (2.7). If $I$ has a $p$-linear free resolution, it coincides with the standard free resolution (see the next section) up to isomorphism.

Recall that a projective scheme $Z \subset \mathbf{P}^{r-1}=\operatorname{Proj}(R)$ has maximal rank if and only if $h^{0}\left(\mathscr{I}_{Z}(t)\right) h^{1}\left(\mathscr{J}_{Z}(t)\right)=0$ for all integers $t$, where $\mathscr{J}_{Z}$ denotes the ideal sheaf of $Z$.

Lemma (2.8) (cf. [A4, (1.11)]). Suppose $c \geq 1$ and let $Z$ denote $\operatorname{Proj}(A)$. Then $Z$ has maximal rank if and only if $c \geq 2$ or $c=1$ and $n_{1}^{1} \leq n_{l}^{r-1} \leq n_{1}^{1}+1$ for all $l\left(1 \leq l \leq m_{r-1}\right)$.

Proof. Note that $H^{0}\left(\mathscr{I}_{Z}(t)\right)=I_{t}, H^{1}\left(\mathscr{I}_{Z}(t)\right) \cong H_{\mathfrak{m}}^{1}(A)_{t}$ for all $t \geq-1$. If $c=\operatorname{depth}_{\mathfrak{m}}(A) \geq 2$, then $H_{\mathrm{m}}^{1}(A)=0$ and we are done. If $c=1$, it follows from (2), (3) of (2.3) that $\max \left\{t \mid h^{1}\left(\mathscr{I}_{Z}(t)\right) \neq 0\right\}=n_{m_{r-1}}^{r-1}-2$. Since $n_{1}^{1}=$ $\min \left\{t \mid h^{0}\left(\mathscr{I}_{Z}(t)\right) \neq 0\right\}$, we get the assertion.

\section{STANDARD FREE RESOlUtions}

Following the fundamental principle described in [A1, Theorem 1.6], one can construct systematically a free resolution of a homogeneous ideal which starts from a system of Weierstrass polynomials. We will analyze the connection between the matrices giving the $p$ th and the $(p+1)$ th modules of syzygies $(p \geq 1)$ obtained by this method in a more general setting.

Let $E$ be an $R$-module which has an expression of the form

$$
E=\bigoplus_{i=1}^{s} \bigoplus_{l=1}^{m_{i}} e_{l}^{i} k[x(i-1)]
$$

with respect to some $x=\left(x_{1}, \ldots, x_{r}\right) \in R_{1}^{\oplus r}$ satisfying $R=k\left[x_{1}, \ldots, x_{r}\right]$, where $s, m_{i}(1 \leq i \leq s)$ are integers such that $1 \leq s \leq r+1, m_{i} \geq 0(1 \leq$ $i \leq s-1), m_{s} \geq 1$, and $e_{l}^{i}\left(1 \leq i \leq s, 1 \leq l \leq m_{i}\right)$ are elements of $E$ different from zero. Note that, unlike the case of homogeneous ideals, we do not necessarily assume $m_{i} \geq 1$ here except for $i=s$. In case $m_{i}=0$, we understand $\bigoplus_{l=1}^{m_{i}}(\cdot)=0$. Let $X_{i}(1 \leq i \leq s-1)$ and $Y_{l}^{i}\left(1 \leq i \leq s, 1 \leq l \leq m_{i}\right)$ 
be letters and set

$$
V=\bigoplus_{i=1}^{s-1} X_{i} R, \quad W^{i}=\bigoplus_{l=1}^{m_{i}} Y_{l}^{i} R \quad(1 \leq i \leq s), \quad W=\bigoplus_{i=1}^{s} W^{i} .
$$

For a sequence $\alpha=\left(\alpha_{1}, \ldots, \alpha_{p}\right)$ of integers with $1 \leq \alpha_{l} \leq s-1(1 \leq l \leq p)$, we denote the element $X_{\alpha_{1}} \wedge \cdots \wedge X_{\alpha_{p}}$ of $\wedge^{p} V$ simply by $X_{\alpha}$. The dual bases associated naturally with the bases $\left\{X_{\alpha}\right\},\left\{Y_{l}^{i}\right\}$ will be denoted by $\left\{X_{\alpha}^{*}\right\},\left\{Y_{l}^{i *}\right\}$ respectively as usual. Put

$$
\varepsilon\left(p ; q, q^{\prime}\right)=\left\{\left(t_{1}, \ldots, t_{p+1}\right) \mid q \leq t_{1}<\cdots<t_{p+1} \leq q^{\prime}\right\} \subset \mathbf{Z}^{p+1}
$$

for a triple $p, q, q^{\prime}$ satisfying $0 \leq p \leq q^{\prime}-q$. The free $R$-module which actually corresponds to the generators of the $p$ th module of syzygies of $E$ constructed by our method is

$$
L_{p}:=\bigoplus_{(\alpha, i) \in \varepsilon(p ; 1, s)} \bigoplus_{l=1}^{m_{i}}\left(X_{\alpha} \otimes Y_{l}^{i}\right) R \subset\left(\bigwedge^{p} V\right) \otimes_{R} W
$$

for each $p(0 \leq p \leq s-1)$. Here $(\alpha, i)$ stands for $\left(\alpha_{1}, \ldots, \alpha_{p}, i\right)$ with $\alpha=$ $\left(\alpha_{1}, \ldots, \alpha_{p}\right) \in \mathbf{Z}^{p}, i \in \mathbf{Z}$ and in the case $p=0$ we understand $\alpha=\varnothing, X_{\varnothing}=1$ for convenience sake. From now on the similar expressions will be used, if there is no fear of confusion. To describe our results clearly we have to distinguish many kinds of subsets of $L_{p}$ from one another. Put

$$
\begin{aligned}
L_{p, q}= & \left\{\bigoplus_{\substack{(\alpha, i) \in \varepsilon(p ; 1, s) \\
\alpha_{1} \leq q+1}} \bigoplus_{l=1}^{m_{i}}\left(X_{\alpha} \otimes Y_{l}^{i}\right) k\left[x\left(\alpha_{1}-1\right)\right]\right\} \\
& \oplus\left\{\bigoplus_{(\alpha, i) \in \varepsilon(p ; q+2, s)} \bigoplus_{l=1}^{m_{i}}\left(X_{\alpha} \otimes Y_{l}^{i}\right) k[x(q)]\right\}, \\
L_{p, q}^{\mathrm{rem}}= & \bigoplus_{(\alpha, i) \in \varepsilon(p ; q+1, s)} \bigoplus_{l=1}^{m_{i}}\left(X_{\alpha} \otimes Y_{l}^{i}\right) k\left[x\left(\alpha_{1}-1\right)\right],
\end{aligned}
$$

for each pair $p, q(0 \leq p \leq s-1,0 \leq q \leq s-p-1)$ and $L_{p}^{\text {rem }}=L_{p, 0}^{\text {rem }}$ for $0 \leq p \leq s-1$, where we understand $\alpha=\varnothing, \alpha_{1}=i$ for $p=0$. The superscript rem is an abbreviation of remainder. Notice that $L_{p}=L_{p, 0} \supseteq \cdots \supseteq L_{p, s-p-1}=$ $L_{p}^{\text {rem }}=L_{p, 0}^{\text {rem }} \supseteq \cdots \supseteq L_{p, s-p-1}^{\text {rem }} \neq 0$. In the following argument we will write an $R$-linear map $\Phi_{p}: L_{p} \rightarrow L_{p-1}(1 \leq p \leq s-1)$ in the form

$$
\Phi_{p}=\sum_{\substack{(\alpha, i) \in \varepsilon(p-1 ; 1, s) \\(\beta, j) \in \varepsilon(p ; 1, s)}}\left(X_{\alpha} \otimes X_{\beta}^{*}\right) \otimes \Phi_{\alpha, \beta}^{i, j}
$$

with $\Phi_{\alpha, \beta}^{i, j} \in \operatorname{Hom}_{R}\left(W^{j}, W^{i}\right)$, and call each $\Phi_{\alpha, \beta}^{i, j}$ a block in $\Phi_{p}$. When $p=1$, the block $\Phi_{\varnothing, \beta}^{i, j}$ will be denoted by $\Phi_{\beta}^{i, j}$. Furthermore, an element of 
$\operatorname{Hom}_{R}\left(W^{j}, W^{i}\right)$ will always be identified with its matrix representation with respect to the bases $\left\{Y_{l}^{j}\right\},\left\{Y_{l}^{i}\right\}$.

Lemma (3.2). Let $\Phi_{p}: L_{p} \rightarrow L_{p-1} \quad(1 \leq p \leq s-1)$ be an $R$-linear map, and suppose that the blocks $\Phi_{\alpha, \beta}^{i, j}((\alpha, i) \in \varepsilon(p-1 ; 1, s),(\beta, j) \in \varepsilon(p ; 1, s))$ in $\Phi_{p}$ satisfy

$$
\left\{\begin{array}{l}
\Phi_{\alpha, \beta}^{i, j} \in \operatorname{MAT}\left(x\left(\alpha_{1}-1\right)\right) \quad \text { for }(\alpha, i) \neq\left(\beta_{2}, \ldots, \beta_{p}, j\right), \\
\Phi_{\alpha,\left(\beta_{1}, \alpha\right)}^{i, i}-x_{\beta_{1}} 1_{m_{i}} \in \operatorname{MAT}\left(x\left(\alpha_{1}-1\right)\right) \quad \text { for } 1 \leq \beta_{1}<\alpha_{1},
\end{array}\right.
$$

where $\alpha=\left(\beta_{2}, \ldots, \beta_{p}\right)=\varnothing$ and $\alpha_{1}=i$ when $p=1$. Then the restriction of $\Phi_{p}$ to $L_{p}^{\mathrm{rem}}$ is injective and we have

$$
L_{p-1, q}=\Phi_{p}\left(L_{p, q}^{\mathrm{rem}}\right) \oplus L_{p-1}^{\mathrm{rem}}
$$

as a $k$-vector space for $0 \leq q \leq s-p-1$, in particular

$$
L_{p-1}=\Phi_{p}\left(L_{p}^{\mathrm{rem}}\right) \oplus L_{p-1}^{\mathrm{rem}} .
$$

Proof. See [A1, Proposition 1.2]. The proof given there works well in our case also, because the number of the terms occurring in a polynomial is finite.

Let us go on to the free resolution of $E$ we are interested in. First we define an $R$-linear map $\Psi_{0}: L_{0} \rightarrow E$ by setting $\Psi_{0}\left(Y_{l}^{i}\right)=e_{l}^{i}$ for all $i, l(1 \leq i \leq$ $\left.s, 1 \leq l \leq m_{i}\right)$. Since $\Psi_{0}\left(L_{0}^{\text {rem }}\right)=E$ and $\left.\Psi_{0}\right|_{L_{0}^{\text {rem }}}$ is injective by (3.1), for every triple $\beta_{1}, j, l$ of integers satisfying $1 \leq \beta_{1}<j \leq s, 1 \leq l \leq m_{j}$, there is a unique $\psi_{\beta_{1}, l}^{j} \in L_{0}^{\text {rem }}$ such that

$$
e_{l}^{j} x_{\beta_{1}}=\Psi_{0}\left(\psi_{\beta_{1}, l}^{j}\right) \text {. }
$$

Let $\Psi_{1}: L_{1} \rightarrow L_{0}$ be the $R$-linear map defined by the formula

$$
\Psi_{1}=\sum_{\left(\beta_{1}, j\right) \in \varepsilon(1 ; 1, s)} \sum_{l=1}^{m_{j}}\left(Y_{l}^{j} x_{\beta_{1}}-\psi_{\beta_{1}, l}^{j}\right) \otimes\left(X_{\beta_{1}}^{*} \otimes Y_{l}^{j *}\right) .
$$

Then apparently this map fulfills the hypothesis of (3.2) and

$$
\operatorname{Ker}\left(\Psi_{0}\right)=\operatorname{Im}\left(\Psi_{1}\right)=\Psi_{1}\left(L_{1}^{\text {rem }}\right)
$$

by [A1, Theorem 1.6]. The same procedure can be carried on successively with the help of the following lemma, until we get the $(s-1)$ th module of syzygies of $E$.

Lemma (3.3). In the situation of (3.2), suppose furthermore that $1 \leq p \leq$ $s-2$ and that $\Phi_{p}\left(L_{p}^{\mathrm{rem}}\right)$ is an $R$-module. Then for every $(\beta, j), l(\beta=$ $\left.\left(\beta_{1}, \ldots, \beta_{p+1}\right),(\beta, j) \in \varepsilon(p+1 ; 1, s), 1 \leq l \leq m_{j}\right)$, there is a unique $\varphi_{\beta, l}^{j} \in L_{p}^{\mathrm{rem}}$ such that

$$
\Phi_{p}\left(X_{\left(\beta_{2}, \ldots, \beta_{p+1}\right)} \otimes Y_{l}^{j}\right) x_{\beta_{1}}=\Phi_{p}\left(\varphi_{\beta, l}^{j}\right)
$$


Moreover the R-linear map $\Phi_{p+1}: L_{p+1} \rightarrow L_{p}$ defined by

$$
\Phi_{p+1}=\sum_{(\beta, j) \in \varepsilon(p+1 ; 1, s)} \sum_{l=1}^{m_{j}}\left(\left(X_{\left(\beta_{2}, \ldots, \beta_{p+1}\right)} \otimes Y_{l}^{j}\right) x_{\beta_{1}}-\varphi_{\beta, l}^{j}\right) \otimes\left(X_{\beta}^{*} \otimes Y_{l}^{j *}\right)
$$

satisfies the hypothesis of (3.2) and

$$
\operatorname{Ker}\left(\Phi_{p}\right)=\operatorname{Im}\left(\Phi_{p+1}\right)=\Phi_{p+1}\left(L_{p+1}^{\mathrm{rem}}\right) .
$$

Proof. By hypothesis, the set $\Phi_{p}\left(L_{p}^{\text {rem }}\right)$ is an $R$-module admitting an expression

$$
\Phi_{p}\left(L_{p}^{\mathrm{rem}}\right)=\bigoplus_{(\alpha, i) \in \varepsilon(p ; 1, s)} \bigoplus_{l=1}^{m_{i}} \Phi_{p}\left(X_{\alpha} \otimes Y_{l}^{i}\right) k\left[x\left(\alpha_{1}-1\right)\right],
$$

which is of the same kind as (3.1). We can therefore apply [A1, Theorem 1.6].

Let $\Psi_{0}, \Psi_{1}$ be as above, and let $\Psi_{p}: L_{p} \rightarrow L_{p-1}(2 \leq p \leq s-1)$ be the $R$-linear maps which are determined by repeated application of (3.2) to $\Psi_{p}(1 \leq p \leq s-2)$ inductively on $p$. Since $L_{s-1}^{\mathrm{rem}}=L_{s-1}$ is a free $R$-module, these maps give a free resolution for $E$ of length $s-1$ :

$$
0 \rightarrow L_{s-1} \stackrel{\Psi_{s-1}}{\longrightarrow} \cdots \rightarrow L_{p} \stackrel{\Psi_{p}}{\longrightarrow} L_{p-1} \rightarrow \cdots \rightarrow L_{1} \stackrel{\Psi_{1}}{\longrightarrow} L_{0} \stackrel{\Psi_{0}}{\longrightarrow} E \rightarrow 0 .
$$

Observe that this resolution is not necessarily minimal but is determined uniquely by the expression (3.1).

Definition (3.4). We call the free resolution of $E$ computed by the algorithm we have described so far the standard free resolution of $E$ starting from $\left\{e_{l}^{i} \mid 1 \leq\right.$ $\left.i \leq s, 1 \leq l \leq m_{i}\right\}$.

The rest of this section is devoted to a detailed description of some general properties of standard free resolutions. For an arbitrary increasing sequence of integers $\delta=\left(\delta_{1}, \ldots, \delta_{p}\right) \quad(p \geq 1)$ and a subsequence $\gamma=\left(\delta_{t_{1}}, \ldots, \delta_{t_{t}}\right)$ of $\delta$, let $\delta \backslash \gamma$ denote the subsequence of $\delta$ obtained by deleting $\delta_{t_{1}}, \ldots, \delta_{t_{l}}$ from $\delta$, where we put $\delta \backslash \varnothing=\delta, \delta \backslash \delta=\varnothing$ for convenience sake. When it is required to pay attention to the indices of the terms that should be deleted, the sequence $\delta \backslash \gamma$ will also be denoted by $\delta\left\{t_{1}, \ldots, t_{l}\right\}$. Given another increasing sequence $\delta^{\prime}=\left(\delta_{1}^{\prime}, \ldots, \delta_{p^{\prime}}^{\prime}\right)\left(p^{\prime} \geq 1\right)$, we define $\eta\left(\delta ; \delta^{\prime}\right)=\left(\delta_{1}, \ldots, \delta_{q}\right)$ with $q=$ $\max \left\{l \mid 1 \leq l \leq p, \delta_{l} \leq \delta_{p^{\prime}}^{\prime}\right\}$ if $\delta_{1} \leq \delta_{p^{\prime}}^{\prime}$ and $\eta\left(\delta ; \delta^{\prime}\right)=\varnothing$ otherwise. Instead of writing a free resolution of a module in the long form as above, we will often denote it by $\Psi_{\bullet}: L_{\bullet} \rightarrow E$.

Theorem (3.5). Let $E$ be an R-module which admits an expression of the form (3.1), $\Psi_{\bullet}: L_{\bullet} \rightarrow E$ the standard free resolution of $E$ starting from $\left\{e_{l}^{i} \mid 1 \leq i \leq\right.$ $\left.s, 1 \leq l \leq m_{i}\right\}$ and $\Psi_{\alpha, \beta}^{i, j}((\alpha, i) \in \varepsilon(p-1 ; 1, s),(\beta, j) \in \varepsilon(p ; 1, s))$ the blocks in $\Psi_{p}(1 \leq p \leq s-1)$. Suppose that $s \geq 3$ and that the blocks in $\Psi_{1}$ satisfy the condition

$$
\Psi_{\beta_{1}}^{i, j} \in \operatorname{MAT}\left(x\left(\beta_{1}\right)\right) \quad \text { for } 1 \leq i \leq \beta_{1}<j \leq s .
$$


Then for every $p(2 \leq p \leq s-1),(\alpha, i) \in \varepsilon(p-1 ; 1, s),(\beta, j) \in \varepsilon(p ; 1, s)$, we have

$$
\Psi_{\alpha, \beta}^{i, j}=(-1)^{q} \operatorname{sgn}\left(\begin{array}{c}
\beta \\
\eta(\alpha ; \beta), \beta \backslash \eta(\alpha ; \beta)
\end{array}\right) \Psi_{\alpha \backslash \eta(\alpha ; \beta), \beta \backslash \eta(\alpha ; \beta)}^{i, j}
$$

with $q$ denoting the number of the terms of $\eta(\alpha ; \beta)$ if $\eta(\alpha ; \beta)$ is a subsequence of $\beta$, and $\Psi_{\alpha, \beta}^{i, j}=0$ otherwise.

The proof will be carried out by induction on $p$. Let $\tau_{p}$ and $\tau_{\beta}^{j} \quad(1 \leq$ $p \leq s-2,(\beta, j) \in \varepsilon(p+1 ; 1, s))$ denote the maps from $\operatorname{Hom}_{R}\left(L_{p}, L_{p-1}\right)$ to $\operatorname{Hom}_{R}\left(L_{p+1}, L_{p}\right)$ given by the formulae

$$
\tau_{\beta}^{j}\left(\Phi_{p}\right)=\sum_{l=1}^{p+1} \sum_{(\gamma, u) \in \varepsilon\left(p-1 ; \beta_{l}+1, s\right)}\left(\left(X_{\beta_{l}} \wedge X_{\gamma}\right) \otimes X_{\beta}^{*}\right) \otimes(-1)^{l} \Phi_{\gamma, \beta\{l\}}^{u, j},
$$

$$
\tau_{p}\left(\Phi_{p}\right)=\sum_{(\beta, j) \in \varepsilon(p+1 ; 1, s)} \tau_{\beta}^{j}\left(\Phi_{p}\right)
$$

for $\Phi_{p} \in \operatorname{Hom}_{R}\left(L_{p}, L_{p-1}\right)$, where the $\Phi_{\gamma, \beta\{l\}}^{u, j}$ 's are the blocks in $\Phi_{p}$.

Lemma (3.7). Let the notation and the assumption be as in (3.5) and fix $t$ $(1 \leq t \leq s-2),(\beta, j) \in \varepsilon(t+1 ; 1, s)$. If $t=1$, or $2 \leq t \leq s-2$ and the conclusion of (3.5) holds for $p=t$, then

$$
\Psi_{t}\left(\tau_{\beta}^{j}\left(\Psi_{t}\right)\left(X_{\beta} \otimes Y_{l}^{j}\right)\right) \in L_{t-1, \min \left(\beta_{t+1}, s-t\right)}
$$

for all $l\left(1 \leq l \leq m_{j}\right)$. If moreover $\beta_{t+1} \geq s-t$ or

$$
\left\{\begin{array}{l}
\Psi_{\gamma, \delta}^{u, v}=0 \text { for all }(\gamma, u) \in \varepsilon(t-1 ; 1, s),(\delta, u) \in \varepsilon(t ; 1, s) \\
\text { satisfying } \gamma_{1}>\max \left(\delta_{2}, \beta_{t+1}+1\right)
\end{array}\right.
$$

then

$$
\Psi_{t}\left(\tau_{\beta}^{j}\left(\Psi_{t}\right)\left(X_{\beta} \otimes Y_{l}^{j}\right)\right) \in L_{t-1}^{\mathrm{rem}}
$$

for all $l\left(1 \leq l \leq m_{j}\right)$, where in the case $t=1$ the condition (3.7.2) should be understood with $\gamma=\varnothing, \gamma_{1}=u, \delta_{2}=v$.

Proof. Let us consider first the case where $2 \leq t \leq s-2$ and the conclusion of (3.5) holds for $p=t$. Since the blocks in the linear maps $\Psi_{t^{\prime}} \quad\left(1 \leq t^{\prime} \leq t\right)$ fulfill (3.2.1), it follows from the hypothesis that

$$
\Psi_{\gamma, \delta}^{u, v} \in \operatorname{MAT}\left(x\left(\max \left(\gamma_{1}-1, \delta_{t}\right)\right)\right)
$$

for all $(\gamma, u) \in \varepsilon(t-1 ; 1, s),(\delta, v) \in \varepsilon(t ; 1, s)$, unless $u=v$ and $\gamma$ is a subsequence of $\delta$. On the other hand

$$
\Psi_{\delta\{l\}, \delta}^{v, v}=(-1)^{l-1} \Psi_{\delta_{l}}^{n, v} \in \operatorname{MAT}\left(x\left(\delta_{l}-1\right)\right)
$$

for all $(\delta, v) \in \varepsilon(t ; 1, s), l \quad(1 \leq l \leq t)$. By (3.6.1)

$$
\Psi_{t} \circ \tau_{\beta}^{j}\left(\Psi_{t}\right)=\sum_{(\alpha, i) \in \varepsilon(t-1 ; 1, s)}\left(X_{\alpha} \otimes X_{\beta}^{*}\right) \otimes \Xi_{\alpha, \beta}^{i, j}
$$


with

$$
\Xi_{\alpha, \beta}^{i, j}:=\sum_{l=1}^{t+1} \sum_{(\gamma, u) \in \varepsilon\left(t-1 ; \beta_{l}+1, s\right)}(-1)^{l} \Psi_{\alpha,\left(\beta_{l}, \gamma\right)}^{i, u} \Psi_{\gamma, \beta\{l\}}^{u, j} \in \operatorname{Hom}_{R}\left(W^{j}, W^{i}\right) .
$$

The properties (3.7.1), (3.7.3) are therefore equivalent to

$$
\begin{aligned}
& \Xi_{\alpha, \beta}^{i, j} \in \operatorname{MAT}\left(x\left(\min \left(\alpha_{1}-1, \beta_{t+1}, s-t\right)\right)\right), \\
& \Xi_{\alpha, \beta}^{i, j} \in \operatorname{MAT}\left(x\left(\alpha_{1}-1\right)\right)
\end{aligned}
$$

for all $(\alpha, i) \in \varepsilon(t-1 ; 1, s)$ respectively. Fix $(\alpha, i) \in \varepsilon(t-1 ; 1, s)$ and put

$$
\begin{aligned}
T^{0}= & \left\{(l, \gamma, u) \mid 1 \leq l \leq t+1,(\gamma, u) \in \varepsilon\left(t-1 ; \beta_{l}+1, s\right)\right\}, \\
T^{1}= & \left\{(l, \alpha, i) \mid 1 \leq l \leq t+1, \beta_{l}<\alpha_{1}\right\} \subset T^{0}, \\
T^{2}= & \left\{(l, \gamma, j) \mid 1 \leq l \leq t+1, \beta_{l}<\gamma_{1}, \gamma \text { is a subsequence of } \beta\{l\}\right\} \subset T^{0}, \\
T^{3}= & T^{0} \backslash\left(T^{1} \cup T^{2}\right), \\
\Xi^{l}= & \sum_{(l, \gamma, u) \in T^{l}}(-1)^{l} \Psi_{\left(\alpha,\left(\beta_{l}, \gamma\right)\right.}^{i, u} \Psi_{\gamma, \beta\{l\}}^{u, j} \quad(0 \leq l \leq 3), \\
\rho= & \min \left(\alpha_{1}-1, \beta_{t+1}\right) .
\end{aligned}
$$

Then $\Xi_{\alpha, \beta}^{i, j}=\Xi^{0}$ and $\max \left(\gamma_{1}-1, \beta\{l\}_{t}\right)=\max \left(\gamma_{1}-1, \beta_{t+1}\right)$ for all $(l, \gamma, u) \in$ $T^{0}$, since $\beta_{t+1}<\gamma_{1}$ in the case $l=t+1$. First of all we find by (3.7.4) and (3.7.5) that $\Psi_{\left(\alpha,\left(\beta_{l}, \gamma\right)\right.}^{i, u} \in \operatorname{MAT}\left(x\left(\alpha_{1}-1\right)\right), \quad \Psi_{\gamma, \beta\{l\}}^{u, j} \in \operatorname{MAT}\left(x\left(\beta_{t+1}\right)\right)$ for all $(l, \gamma, u) \in T^{3}$, which implies that $\Xi^{3} \in \operatorname{MAT}(x(\rho))$. When the condition (3.7.2) holds additionally, for all $(l, \gamma, u) \in T^{3}$ the blocks satisfy

$$
\Psi_{\gamma, \beta\{l\}}^{u, j} \in \operatorname{MAT}\left(x\left(\max \left(\gamma_{1}-1, \beta_{t+1}\right)\right)\right) \subset \operatorname{MAT}\left(x\left(\alpha_{1}-1\right)\right)
$$

if $\alpha_{1} \leq \max \left(\gamma_{1}, \beta_{t+1}+1\right)$ and $\Psi_{\alpha,\left(\beta_{l}, \gamma\right)}^{i, u}=0$ otherwise. Hence

$$
\Xi^{3} \in \operatorname{MAT}\left(x\left(\alpha_{1}-1\right)\right)
$$

in this case. Observe that

$$
\Xi^{1}=\sum_{l=1}^{w}(-1)^{l} \Psi_{\beta_{l}}^{i, i} \Psi_{\alpha, \beta\{l\}}^{i, j}
$$

with $w:=\max \left\{l \mid 1 \leq l \leq t+1, \beta_{l}<\alpha_{1}\right\} \cup\{0\}$ and that

$$
\begin{aligned}
\Xi^{2} & =-\sum_{l=1}^{t} \Psi_{\alpha, \beta\{l+1\}}^{i, j} \Psi_{\beta\{1, l+1\}, \beta\{1\}}^{j, j}+\Psi_{\alpha, \beta\{1\}}^{i, j} \Psi_{\beta\{1,2\}, \beta\{2\}}^{j, j} \\
& =\sum_{l=1}^{t+1}(-1)^{l-1} \Psi_{\alpha, \beta\{l\}}^{i, j} \Psi_{\beta,}^{j, j},
\end{aligned}
$$


where $\Xi^{1}=0$ in the case $w=0$ i.e. $T^{1}=\varnothing$. Put

$$
\dot{\leftrightarrow}_{\delta_{1}}^{v, v}=x_{\delta_{1}} 1_{m_{v}}-\Psi_{\delta_{1}}^{v, v}
$$

for $1 \leq \delta_{1}<v \leq s$. We consider the difference $\Xi:=\Xi_{\alpha, \beta}^{i, j}-\Xi^{3}$, separating two cases.

Case (1). Suppose $T^{1} \cap T^{2}=\varnothing$. Then

$$
\begin{aligned}
\Xi= & \Xi^{1}+\Xi^{2} \\
= & \sum_{l=1}^{w}(-1)^{l-1}\left(\dot{\leftrightarrow}_{\beta_{1}}^{i, i} \Psi_{\alpha, \beta\{l\}}^{i, j}-\Psi_{\alpha, \beta\{l\}}^{i, j} \dot{\leftrightarrow}_{\beta_{1}}^{j, j}\right) \\
& +\sum_{l=w+1}^{t+1}(-1)^{l-1} \Psi_{\alpha, \beta\{l\}}^{i, j} \Psi_{\beta_{l}}^{j, j} .
\end{aligned}
$$

Since the assumption $T^{1} \cap T^{2}=\varnothing$ implies $i \neq j$ or $\alpha \neq \beta\{l\}\{1\}$ for any $l$ $(1 \leq l \leq t+1)$, the blocks $\Psi_{\alpha, \beta\{l\}}^{i, j}(1 \leq l \leq t+1)$ lie in $\operatorname{MAT}\left(x\left(\alpha_{1}-1\right)\right)$ by (3.7.4) and (3.7.5). Besides, $\dot{\Psi}_{\beta_{1}}^{i, i} \in \operatorname{MAT}\left(x\left(\alpha_{1}-1\right)\right), \dot{\leftrightarrow}_{\beta_{1}}^{j, j} \in \operatorname{MAT}\left(x\left(\beta_{t+1}\right)\right)$ for $1 \leq l \leq w$ and $\Psi_{\beta_{l}}^{j, j} \in \operatorname{MAT}\left(x\left(\alpha_{1}-1\right)\right)$ for $w+1 \leq l \leq t+1$ by the conditions $\alpha_{1}<i, \beta_{t+1}<j$ and (3.2.1). Hence $\Xi \in \operatorname{MAT}(x(\rho))$. When (3.7.2) is fulfilled, we have $\Psi_{\alpha, \beta\{l\}}^{i, j} \stackrel{\circ}{\Psi}_{\beta_{1}}^{j, j}=0$ for $1 \leq l \leq w$ if $\alpha_{1}>\beta_{t+1}+1=\max \left(\beta\{l\}_{2}, \beta_{t+1}+1\right)$, therefore $\Xi \in \operatorname{MAT}\left(x\left(\alpha_{1}-1\right)\right)$.

Case (2). Suppose $T^{1} \cap T^{2} \neq \varnothing$. Then $i=j$ and $\alpha$ is a subsequence of $\beta\{l\}$ for some $l(1 \leq l \leq t+1)$ such that $\beta_{l}<\alpha_{1}$. This is possible only when $T^{1} \subset T^{2}$ and $\alpha=\beta\{1, n\}$ with some $n(2 \leq n \leq t+1)$. In this case

$$
\begin{aligned}
\Xi & =\Xi^{2}=\Psi_{\beta\{1, n\}, \beta\{1\}}^{i, i} \Psi_{\beta_{1}}^{i, i}+(-1)^{n-1} \Psi_{\beta\{1, n\}, \beta\{n\}}^{i, i} \Psi_{\beta_{n}}^{i, i}+\Xi^{\prime} \\
& =(-1)^{n}\left(\dot{\Psi}_{\beta_{n}}^{i, i} \dot{\Psi}_{\beta_{1}}^{i, i}-\dot{\Psi}_{\beta_{1}}^{i, i} \dot{\Psi}_{\beta_{n}}^{i, i}\right)+\Xi^{\prime}
\end{aligned}
$$

by (3.7.5) and (3.7.6), where

$$
\Xi^{\prime}=\sum_{\substack{l=1 \\ l \neq 1, n}}^{t+1}(-1)^{l-1} \Psi_{\beta\{1, n\}, \beta\{l\}}^{i, i} \Psi_{\beta_{l}}^{i, i} .
$$

Since $\beta\{1, n\}$ is not a subsequence of $\beta\{l\}$ and $\alpha_{1}=\beta\{1, n\}_{1} \leq \beta_{l}$ for $l \neq 1, n$, the matrix $\Xi^{\prime}$ lies in $\operatorname{MAT}\left(x\left(\alpha_{1}-1\right)\right)$ by (3.2.1) and (3.7.4). On the other hand, since $\alpha_{1}<i$, it is clear that $\Xi-\Xi^{\prime} \in \operatorname{MAT}\left(x\left(\alpha_{1}-1\right)\right)$ by (3.2.1). Hence $\Xi \in \operatorname{MAT}\left(x\left(\alpha_{1}-1\right)\right)$.

Thus for each $(\alpha, i) \in \varepsilon(t-1 ; 1, s)$ the matrix $\Xi_{\alpha, \beta}^{i, j}$ satisfies $(3.7 .1)^{\prime}$, and if moreover the condition (3.7.2) is fulfilled, it also satisfies the stronger $(3.7 .3)^{\prime}$. Since $L_{t-1, \min \left(\beta_{t+1}, s-t\right)}=L_{t-1}^{\mathrm{rem}}$ if $\beta_{t+1} \geq s-t$, this concludes the proof of our lemma for the case $2 \leq t \leq s-2$. 
When $t=1$, we have

$$
\Psi_{1} \circ \tau_{\beta}^{j}\left(\Psi_{1}\right)=\sum_{i=1}^{s}\left(1 \otimes X_{\beta}^{*}\right) \otimes \Xi_{\beta}^{i, j}
$$

with

$$
\Xi_{\beta}^{i, j}=\sum_{l=1}^{2} \sum_{u=\beta_{l}+1}^{s}(-1)^{l} \Psi_{\beta_{l}}^{i, u} \Psi_{\beta\{l\}}^{u, j}
$$

and the properties $(3.7 .1),(3.7 .3)$ are equivalent to

$$
\begin{aligned}
& \Xi_{\beta}^{i, j} \in \operatorname{MAT}\left(x\left(\min \left(i-1, \beta_{2}, s-t\right)\right)\right), \\
& \Xi_{\beta}^{i, j} \in \operatorname{MAT}(x(i-1))
\end{aligned}
$$

for all $i(1 \leq i \leq s)$ respectively. In this case, the matrix corresponding to $\Xi^{3}$ defined in the course of the above proof coincides with the sum of all the terms occurring in $\Xi_{\beta}^{i, j}$ such that $u \neq i, j$. We denote this matrix by the same symbol $\Xi^{3}$. Now apply the same reasoning as above to $\Xi^{3}$ and $\Xi:=\Xi_{\beta}^{i, j}-\Xi^{3}$ with the use of (3.2.1) and (3.5.1). As for $\Xi$, it may be easier to understand if we verify the desired result directly separating the four cases (1) $i \leq \beta_{1}<\beta_{2}<j$, (2) $\beta_{1}<i \leq \beta_{2}<j$, (3) $\beta_{1}<i, \beta_{2}<j, i \neq j$, (4) $\beta_{2}<j=i$. The details are left to the readers. Q.E.D.

Lemma (3.8). Let the notation and the assumption be as in (3.5), fix $t \quad(1 \leq$ $t \leq s-2)$ and let $\tau_{t}\left(\Psi_{t}\right)_{\alpha, \beta}^{i, j}((\alpha, i) \in \varepsilon(t ; 1, s),(\beta, j) \in \varepsilon(t+1 ; 1, s))$ be the blocks in $\tau_{t}\left(\Psi_{t}\right)$. Suppose that $t=1$ or that $2 \leq t \leq s-2$ and the conclusion of (3.5) holds for $p=t$. Then for $(\alpha, i) \in \varepsilon(t ; 1, s),(\beta, j) \in \varepsilon(t+1 ; 1, s)$, we have

$$
\Psi_{\alpha, \beta}^{i, j}=\tau_{t}\left(\Psi_{t}\right)_{\alpha, \beta}^{i, j} \quad \text { if } \alpha_{1} \leq \beta_{t+1} \text { or } \beta_{t+1} \geq s-t
$$

If moreover

$$
\left\{\begin{array}{l}
\Psi_{\gamma, \delta}^{u, v}=0 \text { for all }(\gamma, u) \in \varepsilon(t-1 ; 1, s),(\delta, v) \in \varepsilon(t ; 1, s) \\
\text { satisfying } \gamma_{1}>\delta_{2},
\end{array}\right.
$$

then $\Psi_{t+1}=\tau_{t}\left(\Psi_{t}\right)$, where in the case $t=1$ the condition (3.8.2) should be understood with $\gamma=\varnothing, \gamma_{1}=u, \delta_{2}=v$.

Proof. Let $(\beta, j)$ be an element of $\varepsilon(t+1 ; 1, s)$ and $l\left(1 \leq l \leq m_{j}\right)$ an integer. If $\beta_{t+1}<s-t$, there is a unique $\theta_{\beta, l}^{j} \in L_{t, \beta_{t+1}}^{\text {rem }}$ such that

$$
\theta_{\beta, l}^{\prime j}:=\Psi_{t}\left(\tau_{\beta}^{j}\left(\Psi_{t}\right)\left(X_{\beta} \otimes Y_{l}^{j}\right)\right)-\Psi_{t}\left(\theta_{\beta, l}^{j}\right) \in L_{t-1}^{\mathrm{rem}}
$$

by (3.7.1) and (3.2), while if $\beta_{t+1} \geq s-t$, the condition (3.8.4) holds with $\theta_{\beta, l}^{j}=0$ by (3.7.3). Since $\Psi_{t-1} \circ \Psi_{t}=0$ and $\left.\Psi_{t-1}\right|_{L_{t-1}^{\text {rem }}}$ is injective by (3.2), 
this $\theta_{\beta, l}^{\prime j}$ must be zero. On the other hand, it follows from (3.7.4), (3.7.5) and (3.6.1) that the map $\tau_{\beta}^{j}\left(\Psi_{t}\right)$ satisfies (3.2.1), in particular

$$
\tau_{\beta}^{j}\left(\Psi_{t}\right)\left(X_{\beta} \otimes Y_{l}^{j}\right)-\left(X_{\left(\beta_{2}, \ldots, \beta_{t+1}\right)} \otimes Y_{l}^{j}\right) x_{\beta_{1}} \in L_{t}^{\mathrm{rem}} .
$$

The relation $\theta_{\beta, l}^{\prime j}=0$ is therefore the one obtained by our algorithm (see (3.3.1)). Set

$$
\Theta_{t+1}=\sum_{(\beta, j) \in \varepsilon(t+1 ; 1, s)} \sum_{l=1}^{m_{j}} \theta_{\beta, l}^{j} \otimes\left(X_{\beta}^{*} \otimes Y_{l}^{j *}\right)
$$

with the $\theta_{\beta, l}^{j}$ 's determined as above. Then $\Psi_{t+1}=\tau_{t}\left(\Psi_{t}\right)-\Theta_{t+1}$ by the construction of $\Psi_{t+1}$ and the blocks in $\Theta_{t+1}$ satisfy $\Theta_{\alpha, \beta}^{i, j}=0$ if $1 \leq \alpha_{1} \leq \beta_{t+1}$ or $\beta_{t+1} \geq s-t$. This proves (3.8.1). In the case where (3.8.2) is fulfilled, the condition (3.7.2) holds automatically, therefore $\theta_{\beta, l}^{j}=0$ for all $(\beta, j), l$ by (3.7.3), that is $\Theta_{t+1}=0$ and $\Psi_{t+1}=\tau_{t}\left(\Psi_{t}\right)$.

Proof of Theorem (3.5). Fix an integer $t(1 \leq t \leq s-2)$ and assume that the blocks in $\Psi_{1}$ satisfy (3.5.1). Assume moreover that $t=1$ or that $2 \leq t \leq s-2$ and the conclusion of $(3.5)$ is true for $p=t$. Under this condition we will prove that the formula in (3.5) holds for all $(\alpha, i) \in \varepsilon(t ; 1, s),(\beta, j) \in \varepsilon(t+1 ; 1, s)$. Since our assertion is trivial for all pairs $(\alpha, i),(\beta, j)$ satisfying $\eta(\alpha ; \beta)=\varnothing$, we have only to consider the case $\eta(\alpha ; \beta) \neq \varnothing$. Let $(\alpha, i) \in \varepsilon(t ; 1, s),(\beta, j) \in$ $\varepsilon(t+1 ; 1, s)$ and suppose $\alpha_{1} \leq \beta_{t+1}$. Put $q=\max \left\{l \mid 1 \leq l \leq t, \alpha_{l} \leq \beta_{t+1}\right\}$. To begin with, it follows from (3.8.1), (3.6.1), (3.6.2) that

$$
\Psi_{\alpha, \beta}^{i, j}=\left\{\begin{array}{l}
(-1)^{l} \Psi_{r\{1\}, \beta\{l\}}^{i, j} \quad \text { if } \alpha_{1}=\beta_{l} \text { for some } l(1 \leq l \leq t+1), \\
0 \quad \text { if } \alpha_{1} \leq \beta_{t+1} \text { but } \alpha_{1} \neq \beta_{l} \text { for any } l(1 \leq l \leq t+1) .
\end{array}\right.
$$

Since $\Psi_{\alpha\{\{1\}, \beta\{l\}}^{i, j}=\Psi_{\alpha \backslash, \alpha_{1}, \beta \backslash \alpha_{1}}^{i, j}$ if $\alpha_{1}=\beta_{l}$, this formula is nothing but what we want when $t=1$. Suppose $2 \leq t \leq s-2$. If $\eta(\alpha ; \beta)=\left(\alpha_{1}, \ldots, \alpha_{q}\right)$ is a subsequence of $\beta$, then $\eta\left(\alpha \backslash \alpha_{1} ; \beta \backslash \alpha_{1}\right)=\left(\alpha_{2}, \ldots, \alpha_{q}\right)$ is a subsequence of $\beta \backslash \alpha_{1}$ and $\left(\alpha \backslash \alpha_{1}\right) \backslash \eta\left(\alpha \backslash \alpha_{1} ; \beta \backslash \alpha_{1}\right)=\alpha \backslash \eta(\alpha ; \beta),\left(\beta \backslash \alpha_{1}\right) \backslash \eta\left(\alpha \backslash \alpha_{1} ; \beta \backslash \alpha_{1}\right)=\beta \backslash \eta(\alpha ; \beta)$, where $\left(\alpha_{2}, \ldots, \alpha_{q}\right)=\varnothing$ for $q=1$. In this case, we find therefore by (3.9) and our assumption that

$$
\begin{aligned}
\Psi_{\alpha, \beta}^{i, j} & =(-1)^{l} \Psi_{r\{\{1\}, \beta\{l\}}^{i, j} \\
& =-\operatorname{sgn}\left(\begin{array}{c}
\beta \\
\alpha_{1}, \beta \backslash \alpha_{1}
\end{array}\right) \Psi_{\left(\alpha \backslash \alpha_{1}, \beta \backslash \alpha_{1}\right.}^{i, j} \\
& =-\operatorname{sgn}\left(\begin{array}{c}
\beta \\
\alpha_{1}, \beta \backslash \alpha_{1}
\end{array}\right)(-1)^{q-1} \operatorname{sgn}\left(\begin{array}{c}
\beta \backslash \alpha_{1} \\
\eta\left(\alpha \backslash \alpha_{1} ; \beta \backslash \alpha_{1}\right), \beta \backslash \eta(\alpha ; \beta)
\end{array}\right) \Psi_{(\alpha \backslash \eta(\alpha ; \beta), \beta \backslash \eta(\alpha ; \beta)}^{i, j} \\
& =(-1)^{q} \operatorname{sgn}\left(\begin{array}{c}
\beta \\
\eta(\alpha ; \beta), \beta \backslash \eta(\alpha ; \beta)
\end{array}\right) \Psi_{\alpha \backslash \eta(\alpha ; \beta), \beta \backslash \eta(\alpha ; \beta)}^{i, j}
\end{aligned}
$$

as desired. On the other hand if $\eta(\alpha ; \beta)$ is not a subsequence of $\beta$, then $\alpha_{1} \neq \beta_{l}$ for any $l$ and $\Psi_{\alpha, \beta}^{i, j}=0$ by (3.9), or $\alpha_{1}=\beta_{l}$ for some $l$ and 
$\Psi_{\alpha, \beta}^{i, j}=(-1)^{l} \Psi_{\alpha \backslash \alpha_{1}, \beta \backslash \alpha_{1}}^{i, j}$ by (3.9) but $\eta\left(\alpha \backslash \alpha_{1} ; \beta \backslash \alpha_{1}\right)$ is not a subsequence of $\beta \backslash \alpha_{1}$. In the latter case, $\Psi_{\alpha \backslash \alpha_{1}, \beta \backslash \alpha_{1}}^{i, j}=0$ by our assumption, hence $\Psi_{\alpha, \beta}^{i, j}=0$. We have thus proved that if $t=1$ or $2 \leq t \leq s-2$ and the conclusion of (3.5) holds for $p=t$, then it also holds for $p=t+1$. This completes the proof of (3.5).

Remark (3.10). In the case $E$ is a homogeneous ideal in $R$, its standard free resolution starting from a basis which has the properties described in (1.1) always satisfies the condition (3.5.1). The proof will be given in the next section (cf. [A1, Remark 2.2]).

Corollary (3.11). Let the notation and the assumption be as in (3.5).

(1) For all $p(1 \leq p \leq s-2)$, we have

$$
\Psi_{p+1}=\tau_{p}\left(\Psi_{p}\right)+\sum_{\substack{(\alpha, i) \in \varepsilon\left(p ; \beta_{p+1}+1, s\right) \\(\beta, j) \in \varepsilon(p+1 ; 1, s) \\ \beta_{p+1}<s-p}}\left(X_{\alpha} \otimes X_{\beta}^{*}\right) \otimes \Psi_{\alpha, \beta}^{i, j} .
$$

(2) Let $\omega^{p} \quad(1 \leq p \leq s-1)$ denote the sequence $(s-p, \ldots, s-1)$. Then

$$
\begin{aligned}
\left.\Psi_{p}\right|_{X_{l, p} \otimes W^{s}}= & \sum_{l=1}^{p}(-1)^{l-1}\left(X_{\omega^{p}\{l\}} \otimes X_{\omega^{p}}^{*}\right) \otimes \Psi_{s-p+l-1}^{s, s} \\
& +(-1)^{p-1}\left(X_{\omega^{p}\{p\}} \otimes X_{\omega^{p}}^{*}\right) \otimes \Psi_{s-1}^{s-1, s}
\end{aligned}
$$

for all $p \quad(2 \leq p \leq s-1)$. In particular, we can determine $\Psi_{s-1}$ completely by $\Psi_{1}$ without computing $\Psi_{2}, \ldots, \Psi_{s-2}$.

(3) $\Psi_{p+1}=\tau_{p}\left(\Psi_{p}\right)$ for all $p((s-1) / 2 \leq p \leq s-2)$ (cf. [A1, Example 2.8]).

(4) If (3.8.2) holds with $t=p_{0}$ for some $p_{0}\left(1 \leq p_{0} \leq s-2\right)$, then for all $p\left(p_{0} \leq p \leq s-2\right)$ the condition (3.8.2) holds with $t=p$ also and $\Psi_{p+1}=\tau_{p}\left(\Psi_{p}\right)$.

(5) If $\Psi_{1} \circ \tau_{1}\left(\Psi_{1}\right)=0$, then $\Psi_{p+1}=\tau_{p}\left(\Psi_{p}\right)$ for all $p(1 \leq p \leq s-2)$.

Proof. (1) Clear by (3.5) and (3.8.1), since $\tau_{p}\left(\Psi_{p}\right)_{\alpha, \beta}^{i, j}=0$ if $\alpha_{1} \geq \beta_{p+1}+1$ and $\beta_{p+1}<s-p$.

(2) An immediate consequence of (3.5).

(3) If $(s-1) / 2 \leq p \leq s-2$, then $s-p \leq p+1 \leq \beta_{p+1}$ for all $(\beta, j) \in$ $\varepsilon(p+1 ; 1, s)$. Hence $\Psi_{p+1}=\tau_{p}\left(\Psi_{p}\right)$ by $(1)$.

(4) We will first show that if (3.8.2) is fulfilled for some $t \quad(1 \leq t \leq s-2)$, then it also holds with $t$ replaced by $t+1$. Suppose that (3.8.2) is true for some $t$ and let $(\gamma, u) \in \varepsilon(t ; 1, s),(\delta, v) \in \varepsilon(t+1 ; 1, s)$. Since $\Psi_{t+1}=\tau_{t}\left(\Psi_{t}\right)$ by (3.5) and (3.8), we have $\Psi_{\gamma, \delta}^{u, v}=(-1)^{l} \Psi_{\gamma\{1\}, \delta\{l\}}^{u, v}$ if $\gamma_{1}=\delta_{l}$ for some $l$ $(1 \leq l \leq t+1)$ and $\Psi_{y, \delta}^{u, v}=0$ othewise. For our purpose it is therefore enough to consider the case $\gamma_{1}=\delta_{l}>\delta_{2}$ for some $l$. In particular we are done for $t=1$. If $2 \leq t \leq s-2$ and $\gamma_{1}=\delta_{l}>\delta_{2}$ for some $l$, then $2<l \leq t+1$, 
$\gamma\{1\}_{1}=\gamma_{2}>\gamma_{1}>\delta_{2}=\delta\{l\}_{2}$, so that $\Psi_{\gamma, \delta}^{u, v}=0$ as desired by assumption. Thus our assertion follows from (3.5) and (3.8) by induction.

(5) Since $\tau_{\beta}^{j}\left(\Psi_{1}\right)$ satisfies $(3.8 .5)$ for all $(\beta, j) \in \varepsilon(2 ; 1, s)$, it follows from the hypothesis and the construction of $\Psi_{2}$ that $\Psi_{2}=\tau_{1}\left(\Psi_{1}\right)$. The blocks in $\Psi_{2}$ therefore satisfy (3.8.2) with $t=2$ by (3.6.1), (3.6.2), hence $\Psi_{p+1}=\tau_{p}\left(\Psi_{p}\right)$ for all $p(1 \leq p \leq s-2)$ by (4).

Example (3.12). (1) Set $E=k=R /\left(x_{1}, \ldots, x_{r}\right), s=r+1, m_{i}=0$ for $1 \leq i \leq r, m_{r+1}=1, e_{1}^{r+1}=1 \in E$. Then $E=1 \cdot k, L_{p}=\left(\bigwedge^{p} V\right) \otimes_{R} W^{r+1} \cong$ $\bigwedge^{p} V(0 \leq p \leq r)$ and $\Psi_{1}=\sum_{\beta_{1}=1}^{r}\left(Y_{1}^{r+1} x_{\beta_{1}}\right) \otimes Y_{1}^{r+1 *} \otimes X_{\beta_{1}}^{*}$. In this case the conditions (3.5.1) and (3.8.2) are trivial for $\Psi_{1}$, so that $\Psi_{p+1}^{\beta_{1}}=\tau_{p}\left(\Psi_{p}\right)$ for all $p(1 \leq p \leq r-1)$ by (4) of (3.11). The standard free resolution of $k$ starting from $\{1\}$ therefore coincides with the usual minimal free resolution of $k$ over $R$ given by the Koszul complex of $R$ with respect to $x_{1}, \ldots, x_{r}$.

(2) Let $E$ be a nontrivial monomial ideal in $R=k\left[x_{1}, \ldots, x_{r}\right]$ and suppose that there are monomials $e_{l}^{i}\left(1 \leq i \leq r-c, 1 \leq l \leq m_{i}\right)$ in $x_{1}, \ldots, x_{r}$ with coefficient 1 such that $e_{l}^{i} \in k\left[x_{1}, \ldots, x_{i}\right] x_{i}$ for all $i, l$ and

$$
E=\bigoplus_{i=1}^{r-c} \bigoplus_{l=1}^{m_{i}} e_{l}^{i} k[x(i-1)]
$$

where $0 \leq c<r, m_{i} \geq 1 \quad(1 \leq i \leq r-c)$. Then for every triple $\beta_{1}, j, l$ satisfying $1 \leq \beta_{1}<j \leq r-c, 1 \leq l \leq m_{j}$, we find $e_{l}^{j} x_{\beta_{1}}=e_{l}^{i} x^{\mu}$ for some $i$ $\left(\beta_{1} \leq i \leq j\right), l^{\prime}\left(1 \leq l^{\prime} \leq m_{i}\right), \mu=\left(0, \ldots, 0, \mu_{\max \left(i, \beta_{1}+1\right)}, \ldots, \mu_{j}, 0, \ldots, 0\right) \in$ $\mathbf{Z}_{0}^{r}$, so that the blocks in $\Psi_{1}$ fulfill the conditions (3.5.1) and (3.8.2). Hence $\Psi_{p+1}=\tau_{p}\left(\Psi_{p}\right)$ for all $p \quad(1 \leq p \leq r-c-2)$ by (4) of (3.11). If moreover $\operatorname{char}(k)=0$ and $E$ is Borel fixed (see (4) of (1.1)), then with the notation above $e_{l}^{j} x_{\beta_{1}} / x_{j} \in E$, namely $\mu_{j} \geq 1$. In this case the components of all the blocks in $\Psi_{p}$ therefore lie in $\mathfrak{m}$ for all $p(1 \leq p \leq r-c-1)$ and the standard free resolution of $E$ starting from $\left\{e_{l}^{i} \mid 1 \leq i \leq r-c, 1 \leq l \leq m_{i}\right\}$ is minimal. Note that a Borel fixed monomial ideal always has a basis as described above (cf. Appendix, [Gr, §2], [HU, Lemma 5.6], [Ga, §4], [A1, (2.1.6)]).

We close this section with a lemma which enables us to get considerable information on $\mathrm{Ext}_{R}^{s-1}(E, R)$ from the standard free resolution.

Lemma (3.13). Notation and assumption being as in (3.5), we have

$$
\begin{aligned}
& \operatorname{Im}^{k[x(s-p-1)]}\left({ }^{t} \Psi_{s-p}^{s, s},{ }^{t} \Psi_{s-p+1}^{s, s}, \ldots,{ }^{t} \Psi_{s-1}^{s, s},{ }^{t} \Psi_{s-1}^{s-1, s}\right) \\
& \quad=\left\{\bigoplus_{l=1}^{p-1} \operatorname{Im}^{k[x(s-p+l-2)]}\left({ }^{t} \Psi_{s-p+l-1}^{s, s}\right)\right\} \oplus \operatorname{Im}^{k[x(s-2)]}\left({ }^{t} \Psi_{s-1}^{s, s},{ }^{t} \Psi_{s-1}^{s-1, s}\right)
\end{aligned}
$$


for all $p \quad(1 \leq p \leq s-1)$ (see Notation). Moreover

$$
\begin{aligned}
& \operatorname{Im}^{k[x(s-2)]}\left({ }^{t} \Psi_{s-1}^{s, s},{ }^{t} \Psi_{s-1}^{s-1, s}\right) \\
& \quad=\operatorname{Im}^{k[x(s-2)]}\left({ }^{t} \Psi_{s-1}^{s, s}\right) \oplus\left\{\sum_{u \geq 0} \operatorname{Im}^{k[x(s-1)]}\left(\left({ }^{t} \dot{\Psi}_{s-1}^{s, s}\right)^{u} \cdot{ }^{t} \Psi_{s-1}^{s-1, s}\right)\right\}
\end{aligned}
$$

as a $k[x(s-1)]$-module, where $\stackrel{\circ}{\Psi}_{s-1}^{s, s}=x_{s-1} 1_{m_{s}}-\Psi_{s-1}^{s, s}$.

Proof. Let $\omega^{p} \quad(1 \leq p \leq s-1)$ be as in (3.11). Set

$$
\begin{aligned}
& Q_{p-1}=X_{\omega^{p-1}} \otimes W^{s} \subset L_{p-1}, \\
& Q_{p-2}^{\prime}=\left\{\bigoplus_{l=2}^{p}\left(X_{\omega^{p}\{1, l\}} \otimes W^{s}\right)\right\} \oplus\left(X_{\omega^{p}\{1, p\}} \otimes W^{s-1}\right) \subset L_{p-2}
\end{aligned}
$$

for $2 \leq p \leq s-1$ and let $\pi_{p-2}: L_{p-2} \rightarrow Q_{p-2}^{\prime}$ denote the natural projection. The matrices representing the composite maps $\left.\pi_{p-2} \circ \Psi_{p-1}\right|_{Q_{p-1}}$ and $\pi_{p-2} \circ$ $\left.\Psi_{p-1}\right|_{X_{s-p} \wedge Q_{p-2}^{\prime}}$, which we denote by $\Upsilon_{p-1}$ and $\Upsilon_{p-1}^{\prime}$ respectively, are made up of the blocks $\Psi_{\omega^{p}\{1, l\}, \omega^{p}\left\{l^{\prime}\right\}}^{i, j}$ in $\Psi_{p-1}$ such that $i, j=s, s-1$ and moreover $\Upsilon_{p-1}^{\prime}$ is a square matrix of size $q_{p-1}:=m_{s-1}+(p-1) m_{s}$. Since $\Psi_{\omega^{p}\{1, l\}, \omega^{p}\{l\}}^{i, i}-$ $x_{s-p} 1_{m_{s}}(i=s-1, l=p$ or $i=s, 2 \leq l \leq p)$ and $\Psi_{\omega^{p}\{1, l\}, \omega^{p}\left\{l^{\prime}\right\}}^{i, j}((i, j)=$ $(s, s-1)$ or $(s-1, s)$ or $\left.s-1 \leq i=j \leq s, l \neq l^{\prime}\right)$ all lie in $\operatorname{MAT}(x(s-p))$ for $2 \leq p \leq s-1$ by $(3.2 .1)$, (3.5.1), (3.7.4), (3.7.5), we find $\Upsilon_{p-1}, \Upsilon_{p-1}^{\prime}-$ $x_{s-p} 1_{q_{p-1}} \in \operatorname{MAT}(x(s-p))$ and

$$
\begin{aligned}
& k[x(s-p-1)]^{m_{s}+q_{p-1}}=\operatorname{Im}^{k[x(s-p-1)]}\left(\begin{array}{c}
t \\
\Upsilon_{p-1} \\
{ }^{t} \Upsilon_{p-1}^{\prime}
\end{array}\right) \\
& \oplus\left\{k[x(s-p-1)]^{m_{s}} \oplus k[x(s-p)]^{q_{p-1}}\right\}
\end{aligned}
$$

by [A1, Proposition 1.2] (cf. Notation). Put

$$
\Upsilon_{p}^{\prime \prime}={ }^{t}\left({ }^{t} \Psi_{s-p}^{s, s},-{ }^{t} \Psi_{s-p+1}^{s, s}, \ldots,(-1)^{p-1 t} \Psi_{s-1}^{s, s},(-1)^{p-1 t} \Psi_{s-1}^{s-1, s}\right) .
$$

Then $\operatorname{Im}\left(\left.\Psi_{p}\right|_{Q_{p}}\right) \subset Q_{p-1} \oplus\left(X_{s-p} \wedge Q_{p-2}^{\prime}\right)$ and $\left.\pi_{p-2} \circ \Psi_{p-1} \circ \Psi_{p}\right|_{Q_{p}}$ is represented by the matrix $\left(\Upsilon_{p-1}, \Upsilon_{p-1}^{\prime}\right) \Upsilon_{p}^{\prime \prime}$ by (2) of (3.11), therefore the identity $\Psi_{p-1} \circ$ $\Psi_{p}=0$ implies

$$
{ }^{t} \Upsilon_{p}^{\prime \prime}\left(\begin{array}{c}
t \\
{ }^{t} \Upsilon_{p-1} \\
{ }_{p-1}^{\prime}
\end{array}\right)=0
$$

Hence

$$
\begin{aligned}
& \operatorname{Im}^{k[x(s-p-1)]}\left({ }^{t} \Upsilon_{p}^{\prime \prime}\right) \\
& \quad=\operatorname{Im}^{k[x(s-p-1)]}\left({ }^{t} \Psi_{s-p}^{s, s}\right) \oplus \operatorname{Im}^{k[x(s-p)]}\left({ }^{t} \Psi_{s-p+1}^{s, s}, \ldots,{ }^{t} \Psi_{s-1}^{s, s},{ }^{t} \Psi_{s-1}^{s-1, s}\right)
\end{aligned}
$$


for $2 \leq p \leq s-1$ by (3.13.1) and [A1, Proposition 1.2]. The first formula follows from this by induction on $p$, since it holds trivially for $p=1$. For the second, see $[\mathrm{A} 2,(2.3 .5)]$. Recall that $\Psi_{s-1}^{s-1, s} \in \operatorname{MAT}(x(s-1))$ by (3.5.1).

\section{VERIFICATION OF THE CONDITION (3.5.1)}

Let $I$ be a nontrivial homogeneous ideal in $R$ with $B(I)=\left(n^{i}\right)_{1 \leq i \leq c}, n^{i}=$ $\left(n_{1}^{i}, \ldots, n_{m_{i}}^{i}\right) \quad\left(c=\operatorname{depth}_{\mathfrak{m}}(R / I)\right)$ and let $\Psi_{\bullet}: L_{\bullet} \rightarrow I$ be the standard free resolution of $I$ starting from the basis $\left\{f_{l}^{i} \mid 1 \leq i \leq r-c, 1 \leq l \leq m_{i}\right\}$ which has the properties (1), (2), (3) of (1.1) with respect to a generic $x \in R_{1}^{\oplus r}$. With the notation of the previous section, we introduce a natural grading into the free $R$-modules $L_{p} \quad(0 \leq p \leq r-c-1)$ by putting $\operatorname{deg}\left(X_{l}\right)=1 \quad(1 \leq l \leq r-c)$, $\operatorname{deg}\left(Y_{l}^{i}\right)=n_{l}^{i}\left(1 \leq i \leq r-c, 1 \leq l \leq m_{i}\right)$. Then

$$
L_{p} \cong \bigoplus_{i=p+1}^{r-c} R\left(-n^{i}-p\right)^{\left(\begin{array}{c}
i-1 \\
p
\end{array}\right)}
$$

as a graded $R$-module for $0 \leq p \leq r-c-1$,

$$
\left\{\begin{array}{l}
L_{0}^{\mathrm{rem}} \cong \bigoplus_{i=1}^{r-c} k[x(i-1)]\left(-n^{i}\right) \\
L_{p}^{\mathrm{rem}} \cong \bigoplus_{t=1}^{r-c-p} \bigoplus_{i=t+p}^{r-c} k[x(t-1)]\left(-n^{i}-p\right)^{\left(\begin{array}{c}
i-t-1 \\
p-1
\end{array}\right)} \\
\quad \text { for } 1 \leq p \leq r-c-1
\end{array}\right.
$$

as $k$-vector spaces and the map $\Psi_{p}$ is a homomorphism of graded modules of degree zero for all $p$. Moreover the length of $\Psi_{\bullet}: L_{\bullet} \rightarrow I$ is exactly the one determined by Auslander-Buchsbaum's theorem, though this resolution is not necessarily minimal in general. When $r-c \geq 2$, the blocks in $\Psi_{1}$ satisfy the condition (3.5.1) in a strengthened form, which we will prove below. To begin with, let us recall the

Division Algorithm (4.3). Fix an $r$-tuple $x=\left(x_{1}, \ldots, x_{r}\right)$ of linear forms of $R$ such that $R=k\left[x_{1}, \ldots, x_{r}\right]$. Let $g_{l}^{i}\left(1 \leq i \leq u, 1 \leq l \leq v_{i}, v_{i} \geq 0\right)$ be arbitrary homogeneous polynomials of $R$ different from zero satisfying

$$
\operatorname{in}\left(x ; g_{l}^{i}\right) k[x(i-1)] \cap \operatorname{in}\left(x ; g_{l^{\prime}}^{i^{\prime}}\right) k\left[x\left(i^{\prime}-1\right)\right]=0
$$

for $(i, l) \neq\left(i^{\prime}, l^{\prime}\right), \quad J=\bigoplus_{i=1}^{u} \bigoplus_{l=1}^{v_{i}} \operatorname{in}\left(x ; g_{l}^{i}\right) k[x(i-1)] \subset R$ and $N$ the linear subspace of $R$ spanned over $k$ by all the monomials not contained in $J$. Then the natural inclusions $J \hookrightarrow R$ and $N \hookrightarrow R$ yield an isomorphism $\sigma_{1}: J \oplus N \rightarrow$ $R$. We define a map $\sigma_{2}: J \oplus N \rightarrow R$ by

$$
\sigma_{2}\left(\sum_{i, l} \operatorname{in}\left(x ; g_{l}^{i}\right) h_{l}^{i}, h^{\prime}\right)=\sum_{i, l} g_{l}^{i} h_{l}^{i}+h^{\prime}
$$


for $h_{l}^{i} \in k[x(i-1)] \quad\left(1 \leq i \leq u, 1 \leq l \leq v_{i}\right), h^{\prime} \in N$ and denote the projections from $J \oplus N$ to $J$ and $N$ by $p r_{J}$ and $p r_{N}$ respectively. Put $\sigma=\sigma_{1}^{-1} \circ \sigma_{2}$ and $\lambda=\mathrm{id}_{J}-\left.\operatorname{pr}_{J} \circ \sigma\right|_{J}$. Since $\lambda(h)=0$ or $h \prec \lambda(h)$ for every $h \in J$ different from zero, the image of an element of $J$ by the map $\lambda^{t}$ is zero for all sufficiently large $t$. It is therefore possible to define a map $\kappa: J \oplus N \rightarrow J \oplus N$ by

$$
\kappa\left(h, h^{\prime}\right)=\left(\left(\mathrm{id}_{J}+\sum_{t=1}^{\infty} \lambda^{t}\right)(h), h^{\prime}-\operatorname{pr}_{N} \circ \sigma \circ\left(\mathrm{id}_{J}+\sum_{t=1}^{\infty} \lambda^{t}\right)(h)\right)
$$

for $h \in J, h^{\prime} \in N$. Direct computations show that $\kappa \circ \sigma=\mathrm{id}_{J \oplus N}, \sigma \circ \kappa=\mathrm{id}_{J \oplus N}$, hence $\sigma_{2}=\sigma_{1} \circ \sigma$ is an isomorphism. In other words,

$$
R=\left\{\bigoplus_{i=1}^{u} \bigoplus_{l=1}^{v_{i}} g_{l}^{i} k[x(i-1)]\right\} \oplus N .
$$

Lemma (4.4). With the notation of (4.3) suppose that

$$
\left\{\begin{array}{l}
\operatorname{in}\left(x ; g_{l}^{i}\right) \in k\left[x_{1}, \ldots, x_{i}\right] x_{i}, \\
g_{l}^{i}-\operatorname{in}\left(x ; g_{l}^{i}\right) \in N
\end{array}\right.
$$

for all $i, l\left(1 \leq i \leq u, 1 \leq l \leq v_{i}\right)$. Then

$$
g_{l}^{j} x_{\beta_{1}} \in\left\{\bigoplus_{l^{\prime}=1}^{v_{\beta_{1}}} g_{l^{\prime}}^{\beta_{1}} k\left[x\left(\beta_{1}\right)\right]\right\} \oplus\left\{\bigoplus_{i=\beta_{1}+1}^{u} \bigoplus_{l^{\prime}=1}^{v_{i}} g_{l^{\prime}}^{i} k[x(i-1)]\right\} \oplus N
$$

for every triple $\beta_{1}, j, l$ satisfying $1 \leq \beta_{1}<j \leq u, 1 \leq l \leq v_{j}$.

Proof. Let $\beta_{1}, j, l$ be a triple satisfying $1 \leq \beta_{1}<j \leq u, 1 \leq l \leq v_{j}$. We will prove first that

$$
\bigoplus_{i=\beta_{1}+1}^{u} \bigoplus_{l^{\prime}=1}^{v_{i}} \operatorname{in}\left(x ; g_{l^{\prime}}^{i}\right) k[x(i-1)] \subset\left\{\bigoplus_{i=\beta_{1}+1}^{u} \bigoplus_{l^{\prime}=1}^{v_{i}} g_{l^{\prime}}^{i} k[x(i-1)]\right\} \oplus N .
$$

Denote the left-hand side of this inclusion relation by $J^{\prime}$ and the right-hand side by $J^{\prime \prime}$. Let $\lambda, \kappa, \sigma_{2}=\sigma_{1} \circ \sigma$ be the maps defined in (4.3). Given an element $\operatorname{in}\left(x ; g_{l^{\prime}}^{i}\right) x^{\mu} \quad\left(\beta_{1}+1 \leq i \leq u, 1 \leq l^{\prime} \leq v_{i}, \mu=\left(0, \ldots, 0, \mu_{i}, \ldots, \mu_{r}\right) \in\right.$ $\mathbf{Z}_{0}^{r}$ ) of $J^{\prime}$, each term occurring in $\left\{\operatorname{in}\left(x ; g_{l^{\prime}}^{i}\right)-g_{l^{\prime}}^{i}\right\} x^{\mu}$ belongs to either $N$ or $J^{\prime}$ by (4.4.1), therefore $\lambda$ maps $J^{\prime}$ into itself. Consequently $J^{\prime}=\sigma_{1} \circ$ $\sigma \circ \kappa\left(J^{\prime}\right) \subset \sigma_{2}\left(\left(\operatorname{id}_{J}+\sum_{t=1}^{\infty} \lambda^{t}\right)\left(J^{\prime}\right)\right)+N \subset \sigma_{2}\left(J^{\prime}\right)+N$, which shows (4.4.2). Write $g_{l}^{j}=\sum_{\nu} a_{\nu} x^{\nu}$. Suppose $a_{\nu} \neq 0$ and $x^{\nu} x_{\beta_{1}}=\operatorname{in}\left(x ; g_{l^{\prime}}^{i}\right) b x^{\mu} \in J$ for some $\nu=\left(\nu_{1}, \ldots, \nu_{r}\right), i \quad(1 \leq i \leq u), l^{\prime}\left(1 \leq l^{\prime} \leq v_{i}\right), b \in k, \mu=$ $\left(0, \ldots, 0, \mu_{i}, \ldots, \mu_{r}\right) \in \mathbf{Z}_{0}^{r}$. Since $\beta_{1}<j$, we see by (4.3.1) and (4.4.1) that $x^{\nu_{1}} \ldots x^{\nu_{\beta_{1}}} \notin J$, therefore (1) $i=\beta_{1}, \mu_{\beta_{1}}=0, \mu=\left(0, \ldots, 0, \mu_{\beta_{1}+1}, \ldots, \mu_{r}\right)$ or (2) $i>\beta_{1}$. In the first case we have $x^{\nu} x_{\beta_{1}}=\operatorname{in}\left(x ; g_{l^{\prime}}^{\beta_{1}}\right) b x^{\mu}=g_{l^{\prime}}^{\beta_{1}} b x^{\mu}+$ $\left\{\operatorname{in}\left(x ; g_{l^{\prime}}^{\beta_{1}}\right)-g_{l^{\prime}}^{\beta_{1}} b b x^{\mu} \in g_{l^{\prime}}^{\beta_{1}} b x^{\mu}+N x^{\mu} \subset g_{l^{\prime}}^{\beta_{1}} b x^{\mu}+J^{\prime}+N\right.$ by (4.4.1), while in the second case, clearly $x^{\nu} x_{\beta_{1}} \in J^{\prime}$. Since $J^{\prime}+N \subset J^{\prime \prime}$ by (4.4.2), this implies that $g_{l}^{j} x_{\beta_{1}} \in\left\{\bigoplus_{l^{\prime}=1}^{v_{\beta_{1}}} g_{l^{\prime}}^{\beta_{1}} k\left[x\left(\beta_{1}\right)\right]\right\} \oplus J^{\prime \prime}$. 
Proposition (4.5). Let the notation be as in the beginning of this section and let $\Psi_{\beta_{1}}^{i, j}\left(1 \leq i \leq r-c,\left(\beta_{1}, j\right) \in \varepsilon(1 ; 1, r-c)\right)$ be the blocks in $\Psi_{1}$. Then

(1) $\Psi_{\beta_{1}}^{i, j}=0$ for $1 \leq i<\beta_{1}$,

(2) $\Psi_{\beta_{1}}^{i, j} \in x(j-1) \operatorname{MAT}\left(x\left(\max \left(i-1, \beta_{1}\right)\right)\right)$ for $\beta_{1} \leq i<j$.

Proof. (1) For each triple $\beta_{1}, j, l \quad\left(1 \leq \beta_{1}<j \leq r-c, 1 \leq l \leq m_{i}\right)$, it follows from (1), (2), (3) of (1.1) and (4.4) that

$$
f_{l}^{j} x_{\beta_{1}}=\sum_{l^{\prime}=1}^{m_{\beta_{1}}} f_{l^{\prime}}^{\beta_{1}} h_{l^{\prime}}^{\beta_{1}}+\sum_{i=\beta_{1}+1}^{r-c} \sum_{l^{\prime}=1}^{m_{i}} f_{l^{\prime}}^{i} h_{l^{\prime}}^{i}
$$

with $h_{l^{\prime}}^{i} \in k\left[x\left(\max \left(i-1, \beta_{1}\right)\right)\right] \quad\left(\beta_{1} \leq i \leq r-c\right)$. Hence $\Psi_{\beta_{1}}^{i, j}=0$ for $1 \leq i<\beta_{1}$ and $\Psi_{\beta_{1}}^{\beta_{1}, j} \in \operatorname{MAT}\left(x\left(\beta_{1}\right)\right)$ by the construction of $\Psi_{1}$.

(2) Note first that $\Psi_{1}$ satisfies (3.2.1). Fix $\left(\beta_{1}, j\right) \in \varepsilon(1 ; 1, r-c)$. Then the identity $\Psi_{0} \circ \Psi_{1}=0$ implies $\sum_{i=1}^{r-c}\left(f_{1}^{i}, \ldots, f_{m_{i}}^{i}\right) \Psi_{\beta_{1}}^{i, j}=0$. Substitute 0 for $x_{t}$ for all $t(j \leq t \leq r)$ in this relation and let $\bar{f}_{l}^{i}$ (resp. $\left.\bar{\Psi}_{\beta_{1}}^{i, j}\right)$ denote the resulting polynomial lying (resp. matrix with components) in $k\left[x_{1}, \ldots, x_{j-1}\right]$. Since $\bar{f}_{l}^{i}=0$ for $j \leq i \leq r-c$, the above relation becomes $\sum_{i=1}^{j-1}\left(\bar{f}_{1}^{i}, \ldots, \bar{f}_{m_{i}}^{i}\right) \bar{\Psi}_{\beta_{1}}^{i, j}=$ 0 , from which follows that $\bar{\Psi}_{\beta_{1}}^{i, j}=0$ for all $i(1 \leq i \leq j-1)$ by (2) of (1.2) and (3.2.1). The components of $\Psi_{\beta_{1}}^{i, j}$ are therefore all contained in $x(j-1) k\left[x\left(\max \left(i-1, \beta_{1}\right)\right)\right]$ for $\beta_{1} \leq i<j$ by (3.2.1) and the last part of the proof of $(1)$.

Corollary (4.6) (cf. [A2, Proposition 2.4]). With the notation above, suppose $0 \leq c \leq r-2$. Then

$$
\begin{aligned}
& \operatorname{Hom}_{k}\left(H_{\mathrm{m}}^{c}(A), k\right)(r) \cong \operatorname{Coker}\left(\Psi_{r-c-1}^{\vee}: L_{r-c-2}^{\vee} \rightarrow L_{r-c-1}^{\vee}\right) \\
& \cong \frac{\bigoplus_{l=1}^{m_{r-c}}\left(\left(X_{1} \wedge \cdots \wedge X_{r-c-1}\right)^{*} \otimes Y_{l}^{r-c *}\right) k[x(r-c-1)]}{\sum_{u \geq 0}\left(X_{1} \wedge \cdots \wedge X_{r-c-1}\right)^{*} \otimes \operatorname{Im}^{k[x(r-c-1)]}\left(\left({ }^{t} \dot{\leftrightarrow}_{r-c-1}^{r-c, r-c}\right)^{u} \cdot{ }^{t} \Psi_{r-c-1}^{r-c-1, r-c}\right)}
\end{aligned}
$$

with $\stackrel{\circ}{\Psi}_{r-c-1}^{r-c, r-c}=x_{r-c-1} 1_{m_{r-c}}-\Psi_{r-c-1}^{r-c, r-c}$, where the second isomorphism stands for that of $k[x(r-c-1)]$-modules. In particular $\operatorname{Hom}_{k}\left(H_{\mathrm{m}}^{c}(A), k\right)$ has a minimal free resolution of the form

$$
\cdots \rightarrow k[x(r-c-1)]\left(n^{r-c}-c-1\right) \rightarrow \operatorname{Hom}_{k}\left(H_{\mathrm{m}}^{c}(A), k\right) \rightarrow 0
$$

as a $k[x(r-c-1)]$-module.

Proof. The first isomorphism is nothing but the local duality. The second follows from (2) of (3.11), (3.13) and the direct sum

$$
R^{m_{r-c}}=\left\{\bigoplus_{l=1}^{r-c-1} \operatorname{Im}^{k[x(l-1)]}\left({ }^{t} \Psi_{l}^{r-c, r-c}\right)\right\} \oplus k[x(r-c-1)]^{m_{r-c}}
$$


The minimality of the generators $\left(X_{1} \wedge \cdots \wedge X_{r-c-1}\right)^{*} \otimes Y_{l}^{r-c *} \quad\left(1 \leq l \leq m_{r-c}\right)$ over $k[x(r-c-1)]$ follows from $(2)$ of $(4.5)$.

\section{Graded BuchSBAUM RINGS}

We will extract from the results of the preceding sections some consequences which characterize the basic sequences of homogeneous ideals defining graded Buchsbaum rings (cf. [SV, Chapter I, Definitions 1.5, 3.1]). In the argument below a crucial role will be played by the structure theorem for maximal Buchsbaum modules over regular local rings due to $\mathrm{S}$. Goto. In the graded case it reads as follows.

Theorem (5.1) ([Go1], [Go2, (3.1)]). Let $S$ be a polynomial ring over $k$ in $u$ indeterminates $z_{1}, \ldots, z_{u}(u \geq 1), \mathfrak{n}$ its maximal ideal $\left(z_{1}, \ldots, z_{u}\right) S,\left(K_{\bullet}(S), \partial_{\bullet}\right)$ the Koszul complex of $S$ with respect to $z=\left(z_{1}, \ldots, z_{u}\right)$ and $\operatorname{Syz}_{j}(k)=$ $K_{j}(S) / \operatorname{Im}\left(\partial_{j+1}\right)$ for $0 \leq j \leq u$. Then a finitely generated graded $S$-module $E$ of dimension $u$ is Buchsbaum if and only if

$$
E \cong \bigoplus_{j=0}^{u} \operatorname{Syz}_{j}^{S}(k)\left(-w^{j}\right)
$$

as a graded $S$-module with suitable sequences of integers $w^{j}=\left(w_{1}^{j}, \ldots, w_{v_{j}}^{j}\right)$ $(0 \leq j \leq u)$, where $w^{j}=\varnothing$ and $\operatorname{Syz}_{j}^{S}(k)\left(-w^{j}\right)=0$ if $v_{j}=0$. Moreover in this case, the sequence $w^{j}$ is determined by the isomorphism $H_{\mathfrak{n}}^{j}(E) \cong k\left(-w^{j}\right)$ up to permutation and $v_{j}=h_{\mathfrak{n}}^{j}(E)$ for each $j \quad(0 \leq j<u)$.

In addition to this theorem we need the following

Lemma (5.2). Let $S=k\left[z_{1}, \ldots, z_{u}\right]$ and $\mathfrak{n}$ be as above and let

$$
0 \rightarrow E \rightarrow G_{u-v} \rightarrow G_{u-v-1} \rightarrow \cdots \rightarrow G_{2} \rightarrow G_{1} \rightarrow M \rightarrow 0
$$

be an exact sequence of finitely generated graded $S$-modules such that $v=$ $\operatorname{dim}(M)<u$ and $G_{p}(1 \leq p \leq u-v)$ are free. Then $H_{\mathrm{n}}^{j}(M) \cong H_{\mathfrak{n}}^{j+u-v}(E)$, $\operatorname{Ext}_{S}^{j}(k, M) \cong \operatorname{Ext}_{S}^{j+u-v}(k, E)$ for $0 \leq j<v, H_{\mathfrak{n}}^{j}(E)=\operatorname{Ext}_{S}^{j}(k, E)=0$ for $0 \leq j<u-v$ and $M$ is a Buchsbaum $S$-module if and only if so is $E$.

Proof. The isomorphisms of cohomology modules are easy to verify. Since we have a commutative diagram

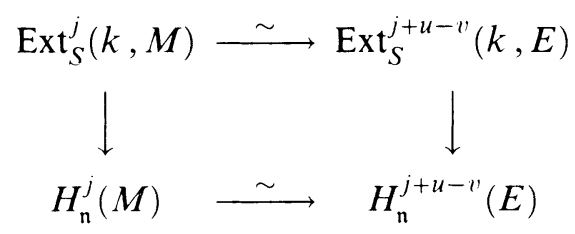

with canonical vertical homomorphisms for all $j(0 \leq j<v)$, the module $M$ is Buchsbaum if and only if so is $E$ by the cohomological characterization of graded Buchsbaum modules (see [SV, Chapter I, Corollary 2.16, Theorem 3.7]). 
Remark (5.3). Note that

$$
\operatorname{Syz}_{j}^{S}(k) \cong \operatorname{Im}\left(\partial_{j}\right) \cong \bigoplus_{t=1}^{u-j+1} k[z(t-1)](-j)^{\left(\begin{array}{c}
u-t \\
j-1
\end{array}\right)} \text { for } 1 \leq j \leq u
$$

by (1) of (3.12) or directly by [A1, Proposition 1.2].

From now on we will fix a nontrivial homogeneous ideal $I \subset R$. Let $x=$ $\left(x_{1}, \ldots, x_{r}\right)$ be a generic element of $R_{1}^{\oplus r}$. Put $A=R / I, c=\operatorname{depth}_{\mathrm{m}}(A), d=$ $\operatorname{dim}(A), B(I)=\left(n^{i}\right)_{1 \leq i \leq r-c}$ with $n^{i}=\left(n_{1}^{i}, \ldots, n_{m_{i}}^{i}\right)$ and $A^{\langle p\rangle}=A / x(r-p) A$ for $0 \leq p \leq d$.

Theorem (5.4). Suppose that $A$ is a graded Buchsbaum ring and let $w^{j}=$ $\left(w_{1}^{j}, \ldots, w_{h_{\mathrm{m}}^{j}(A)}^{j}\right)$ be a sequence of integers such that $H_{\mathfrak{m}}^{j}(A) \cong k\left(-w^{j}\right)$ for each $j(c \leq j<d)$. Then d) ,

(1) $n^{r-p}=\left(\left(w^{j}+j+1\right)^{\left(\begin{array}{l}p \\ j\end{array}\right)}\right)_{c \leq j \leq p}$ up to permutation for every $p \quad(c \leq p<$

(2) $\left(\left(w^{j}+j+1\right)^{\left(\begin{array}{c}d \\ j\end{array}\right)}\right)_{c \leq j<d}$ is a subsequence of $n^{r-d}$ up to permutation.

Proof. (1) For each $p(0 \leq p<d)$ the ring $A^{\langle p\rangle}$ is also Buchsbaum and $\mathfrak{m} H_{\mathfrak{m}}^{0}\left(A^{\langle p\rangle}\right)=0$, therefore

$$
H_{\mathrm{m}}^{0}\left(A^{\langle p\rangle}\right) \cong k\left(-n^{r-p}+1\right)
$$

for $c \leq p<d$ by (2) of (1.2) and (2.1). On the other hand, since $x_{r-q} H_{\mathfrak{m}}^{j}\left(A^{\langle q\rangle}\right)$ $=0$ for all $j, q(0 \leq q<d, 0 \leq j<d-q)$, we find

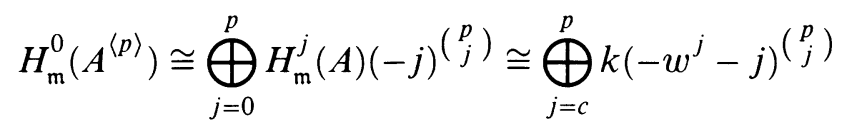

for $c \leq p<d$, applying (2.2) to $A^{\langle q\rangle}$ and its parameter $x_{r-q}$ for $0 \leq q<d-1$. Compare (5.4.1) and (5.4.2).

(2) We denote the ring $k[x(r-d-1)]$ by $S$ and its maximal ideal $x(r-d-1) S$ by $\mathfrak{n}$. Let $\left\{f_{l}^{i} \mid 1 \leq i \leq r-c, 1 \leq l \leq m_{i}\right\}$ be as in (1.1) and let $J$ denote the $S$-module $\bigoplus_{i=1}^{r-d-1} \bigoplus_{l=1}^{m_{i}} f_{l}^{i} k[x(i-1)]$. Consider the exact sequence

$$
0 \rightarrow I / J \rightarrow R / J \rightarrow A \rightarrow 0
$$

of graded $S$-modules. Since $l_{S}\left(R / J \otimes_{S} S / \mathfrak{n}\right)=l_{R}(R / I+x(r-d-1) R)<\infty$ by (2) of (1.2), the $S$-module $R / J$ is finitely generated and free by (3) of (1.1) and (1) of (1.3). Moreover $A$ is a Buchsbaum $S$-module by hypothesis. It follows therefore from (5.2) that $I / J$ is a Buchsbaum $S$-module such that $H_{\mathfrak{m}}^{j}(A) \cong H_{\mathfrak{n}}^{j}(A) \cong H_{\mathfrak{n}}^{j+1}(I / J)$ for all $j(0 \leq j<d)$ and $H_{\mathfrak{n}}^{0}(I / J)=0$. Consequently

$$
I / J \cong\left\{\bigoplus_{j=c}^{d-1} \operatorname{Syz}_{j+1}^{S}(k)\left(-w^{j}\right)\right\} \oplus S(-w)
$$


by (5.1), where $w$ is a suitable sequence of integers. This isomorphism and (5.3) yield

$$
\begin{aligned}
& I / J \cong\left\{\bigoplus_{j=c}^{d-1} \bigoplus_{p=j}^{d} k[x(r-p-1)]\left(-w^{j}-j-1\right)^{\left.\left(\begin{array}{c}
p \\
j
\end{array}\right)\right\}} \oplus S(-w)\right. \\
&=\left\{\bigoplus_{p=c}^{d-1} \bigoplus_{j=c}^{p} k[x(r-p-1)]\left(-w^{j}-j-1\right)^{\left(\begin{array}{c}
p \\
j
\end{array}\right)}\right\} \\
& \oplus\left\{\bigoplus_{j=c}^{d-1} S\left(-w^{j}-j-1\right)^{\left(\begin{array}{c}
d \\
j
\end{array}\right)}\right\} \oplus S(-w),
\end{aligned}
$$

while the formula (1.1.1) implies

$$
I / J \cong S\left(-n^{r-d}\right) \oplus\left\{\bigoplus_{p=c}^{d-1} k[x(r-p-1)]\left(-n^{r-p}\right)\right\}
$$

With the use of these expressions for $I / J, \operatorname{dim}_{k}(I / J)_{t}$ can be computed in two ways and by (1) we find

$$
\operatorname{dim}_{k}\left(S\left(-n^{r-d}\right)\right)_{t}=\sum_{j=c}^{d-1} \operatorname{dim}_{k}\left(S\left(-w^{j}-j-1\right)^{\left(\begin{array}{c}
d \\
j
\end{array}\right)}\right)_{t}+\operatorname{dim}_{k}(S(-w))_{t}
$$

for all $t \in \mathbf{Z}$. Hence $n^{r-d}=\left(\left(\left(w^{j}+j+1\right)^{\left(\begin{array}{l}d \\ j\end{array}\right)}\right)_{c \leq j<d}, w\right)$ up to permutation.

Corollary (5.5). Let $\Psi_{\bullet}: L_{\bullet} \rightarrow I$ be the standard free resolution of $I$ starting from the basis described in (1.1). If $A$ is Buchsbaum, then $\operatorname{Im}\left(\Psi_{r-d-1}\right)$ is a graded Buchsbaum $R$-module and the maps $\Psi_{p+r-d-1}(0 \leq p \leq d-c)$ yield a minimal free resolution of $\operatorname{Im}\left(\Psi_{r-d-1}\right)$.

Proof. Let $E$ denote $\operatorname{Im}\left(\Psi_{r-d-1}\right)$ and let $\Phi_{\bullet}: F_{\bullet} \rightarrow E$ be a minimal free resolution of $E$. It follows from the hypothesis and (5.2) that $E$ is a graded Buchsbaum $R$-module and moreover $E$ has another free resolution $\Psi_{\bullet+r-d-1}$ : $L_{\bullet+r-d-1} \rightarrow E$ given by the maps $\Psi_{p+r-d-1}(0 \leq p \leq d-c)$. With the notation of (5.4), $E$ is isomorphic to the direct sum of

$$
\bigoplus_{j=c}^{d-1} \operatorname{Syz}_{j+r-d}^{R}(k)\left(-w^{j}\right)
$$


and a free $R$-module by $(5.1),(5.2)$, so that $F_{p}=0$ for $p>d-c$ and $\operatorname{rank}_{R}\left(F_{p}\right)=\sum_{j=c}^{d-p}\left(\begin{array}{c}r \\ j+p+r-d\end{array}\right) h_{\mathfrak{m}}^{j}(A)$ for $1 \leq p \leq d-c$. On the other hand

$$
\begin{aligned}
\operatorname{rank}_{R}\left(L_{p+r-d-1}\right) & =\sum_{i=p+r-d}^{r-c}\left(\begin{array}{c}
i-1 \\
p+r-d-1
\end{array}\right) m_{i} \\
& =\sum_{i=p+r-d}^{r-c} \sum_{j=c}^{r-i}\left(\begin{array}{c}
i-1 \\
p+r-d-1
\end{array}\right)\left(\begin{array}{c}
r-i \\
j
\end{array}\right) h_{\mathfrak{m}}^{j}(A) \\
& =\sum_{j=c}^{d-p} \sum_{i=p+r-d}^{r-j}\left(\begin{array}{c}
i-1 \\
p+r-d-1
\end{array}\right)\left(\begin{array}{c}
r-i \\
j
\end{array}\right) h_{\mathfrak{m}}^{j}(A) \\
& =\sum_{j=c}^{d-p}\left(\begin{array}{c}
r \\
j+p+r-d
\end{array}\right) h_{\mathfrak{m}}^{j}(A)
\end{aligned}
$$

for all $p(1 \leq p \leq d-c)$ by (4.1), (1) of (5.4) and an elementary formula

$$
\sum_{t=u}^{\prime \prime-u^{\prime}}\left(\begin{array}{l}
t \\
u
\end{array}\right)\left(\begin{array}{c}
v-t \\
u^{\prime}
\end{array}\right)=\left(\begin{array}{c}
v+1 \\
u+u^{\prime}+1
\end{array}\right) \quad\left(u, u^{\prime} \geq 0, v \geq u+u^{\prime}\right) .
$$

Hence $\operatorname{dim}_{k}\left(\operatorname{Tor}_{p}^{R}(k, E)\right)=\operatorname{rank}_{R}\left(F_{p}\right)=\operatorname{rank}_{R}\left(L_{p+r-d-1}\right)$ for $1 \leq p \leq d-c$, which implies our assertion.

Corollary (5.6). Suppose $A$ is Buchsbaum. Then

$$
m_{r-d} \geq \sum_{j=0}^{d-1}\left(\begin{array}{l}
d \\
j
\end{array}\right) h_{\mathrm{m}}^{j}(A)
$$

in particular

(1) $c=0, h_{\mathrm{m}}^{0}(A)=1, h_{\mathrm{m}}^{j}(A)=0(0<j<d)$ if $r-d=1, c<d$,

(2) $\min \left\{t \mid I_{t} \neq 0\right\} \geq \sum_{j=0}^{d-1}\left(\begin{array}{l}d \\ j\end{array}\right) h_{\mathrm{m}}^{j}(A)$ if $r-d=2$.

Proof. An immediate consequence of (2) of (5.4). Recall that $m_{1}=1, m_{2}=$ $n_{1}^{1}=\min \left\{t \mid I_{t} \neq 0\right\} \quad($ see $(1)$ of $(1.6)$ and of $(1.7))$.

Corollary (5.7) (cf. [EF, §5], [GM1, §3]). Suppose that $c \geq 1$ and that $Z:=$ $\operatorname{Proj}(A)$ is an arithmetically Buchsbaum subscheme of $\mathbf{P}^{r-1}$ with $\operatorname{dim}(Z) \geq 1$ which has maximal rank. Then $h^{1}\left(\mathscr{F}_{Z}(t)\right)=0$ for all integers $t$ or

$$
n_{1}^{1}-2 \leq \min \left\{t \mid h^{1}\left(\mathscr{I}_{Z}(t)\right) \neq 0\right\} \leq \max \left\{t \mid h^{1}\left(\mathscr{I}_{Z}(t)\right) \neq 0\right\} \leq n_{1}^{1}-1,
$$

where $\mathscr{I}_{Z}$ denotes the ideal sheaf of $Z$.

Proof. If $c=1$, then $n^{r-1}=w^{1}+2$ up to permutation by (5.4), where $w^{1}$ is a sequence of integers such that $\bigoplus_{t} H^{1}\left(\mathscr{J}_{Z}(t)\right) \cong k\left(-w^{1}\right)$. We therefore get the inequality by $(2.8)$.

Remark (5.8). (1) The above results (5.4), (5.5), (5.6) generalize the essence of the structure theorem for arithmetically Buchsbaum curves in $\mathbf{P}^{3}$ proved in 
my previous work [A2]. There the case $1 \leq c \leq d=2, r=4$ was treated exclusively. Recently M. Chang in [C] gave another structure theorem for the case $1 \leq c \leq d=r-2, r \geq 4$, with the use of which she obtained the inequality stated in (2) of (5.6) (cf. (7.1), (7.2)). Note that our generalization above includes the case $c=0$ also.

(2) We will show in the next section that the lower bound estimate for the minimal degree of the generators of $I$ described in (2) of (5.6) is sharp. This estimate, however, does not hold when $r-d \geq 3$, as seen by the following example. Put $c=1, d=2, r=5$ and let $I$ be the ideal in $R$ generated by $x_{1}^{2}, x_{1} x_{2}^{u} x_{3}^{\prime \prime}(u+v=t), x_{2}^{u} x_{3}^{\prime \prime} \quad(u+v=t+1), x_{1} x_{2}^{u} x_{3}^{v-1} x_{4}-x_{2}^{u+1} x_{3}^{v-1} x_{5}$ $(u+v=t, v \geq 1)$ for $t \geq 1$. Then $H_{\mathrm{m}}^{0}(A)=0, H_{\mathrm{m}}^{1}(A) \cong k(-t+1)^{t}$ and $A$ is Buchsbaum with $n_{1}^{1}=2, \sum_{j=0}^{1}\left(\begin{array}{l}2 \\ j\end{array}\right) h_{\mathrm{m}}^{j}(A)=2 t$.

(3) When $A$ is Buchsbaum with $c=1, d=2, r=4$, there is a sequence $w$ such that $n^{2}=\left(n^{3}, n^{3}, w\right)$ up to permutation by (5.4). Let $n^{3}\{1\}$ denote $\left(n_{2}^{3}, \ldots, n_{m_{3}}^{3}\right)$. Then the numerical character of $\operatorname{Proj}(A)$ is $\left(n^{3}, n^{3}-1, w\right)$ if $n_{1}^{3}>n_{1}^{1}$ and $\left(n^{3}, n^{3}\{1\}-1, w\right)$ if $n_{1}^{3}=n_{1}^{1}$, up to permutation (cf. (2) of (1.7)).

Next we will give a necessary and sufficient condition for $A$ to be a graded Buchsbaum ring in terms of the invariants $m_{r-d+1}$ and $h_{\mathrm{m}}^{j}(A) \quad(0 \leq j<d)$.

Lemma (5.9). Let $M$ be a finitely generated graded $R$-module of dimension $v$ and $z_{1}, \ldots, z_{v}$, a homogeneous system of parameters for $M$. Then

$$
h_{\mathfrak{m}}^{q}\left(M /\left(z_{1}, \ldots, z_{p}\right) M\right) \leq \sum_{j=0}^{p}\left(\begin{array}{c}
p \\
j
\end{array}\right) h_{\mathfrak{m}}^{q+j}(M)
$$

for each pair $p, q$ satisfying $p \geq 0,0 \leq p+q<v$. The equality holds if and only if $z_{t+1} H_{\mathrm{m}}^{q+j}\left(M /\left(z_{1}, \ldots, z_{t}\right) M\right)=0$ for all $j, t$ such that $j \geq 0,0 \leq t<p$, $0 \leq j+t \leq p$.

Proof. An immediate consequence of the long exact sequences of the form (2.2) applied to the pairs $M /\left(z_{1}, \ldots, z_{t}\right) M, z_{t+1}(0 \leq t<p)$ instead of $A, x_{r}$.

Lemma (5.10). Let $M$ be as above with $v \geq 1$ and $z_{1}, \ldots, z_{v}$ a homogeneous system of parameters for $M$ consisting of linear forms. Then

$$
l_{R}\left(M /\left(z_{1}, \ldots, z_{v}\right) M\right)-\operatorname{deg}(M) \leq \sum_{j=0}^{v-1}\left(\begin{array}{c}
v-1 \\
j
\end{array}\right) h_{\mathfrak{m}}^{j}(M)
$$

with equality if and only if $z_{t+1} H_{\mathrm{m}}^{j}\left(M /\left(z_{1}, \ldots, z_{t}\right) M\right)=0$ for all $j, t$ such that $j \geq 0, t \geq 0,0 \leq j+t<v$.

Proof. The module $M^{\prime \prime}:=M /\left(z_{1}, \ldots, z_{v-1}\right) M$ fulfills

$$
l_{R}\left(M^{\prime \prime} / z_{v} M^{\prime \prime}\right)-\operatorname{deg}\left(M^{\prime \prime}\right)=l_{R}\left(0:_{M^{\prime \prime}} z_{v}\right) \leq h_{\mathfrak{m}}^{0}\left(M^{\prime \prime}\right)
$$

with equality if and only if $z_{v} H_{\mathrm{m}}^{0}\left(M^{\prime \prime}\right)=0$ by [AB, Corollary 4.3]. This together with (5.9) shows what we want. 
Proposition (5.11). Let $M$ be a finitely generated graded $R$-module of dimension $v \geq 1$ and suppose that there is a Zariski open subset $U_{M}$ of $R_{1}^{\oplus v}$ such that $l_{R}\left(M /\left(z_{1}, \ldots, z_{v}\right) M\right)<\infty$ and the equality holds in $(5.10 .1)$ for all $\left(z_{1}, \ldots, z_{v}\right)$ $\in U_{M}$. Then $M$ is a Buchsbaum R-module.

Proof. The hypothesis implies that $z_{1} H_{\mathrm{m}}^{j}(M)=0$ for all generic $z_{1}$ and $j(0 \leq$ $j<v)$ by $(5.10)$, therefore $\mathfrak{m} H_{\mathfrak{m}}^{j}(M)=0$ for all $j(0 \leq j<v)$, in particular $M$ is a Buchsbaum $R$-module if $v=1$. Suppose that $v \geq 2$ and that the assertion is true for modules of smaller dimensions. Let $g$ be a homogeneous polynomial of positive degree and $\left(z_{1}, \ldots, z_{v}\right)$ an arbitrary element of $U_{M}$ such that $z_{1}, \ldots, z_{v-1}, g$ is a system of parameters for $M$. Set $M^{\prime}=M / g M, M^{\prime \prime}=$ $M /\left(z_{1}, \ldots, z_{v-1}\right) M$. Since $z_{v} H_{\mathrm{m}}^{0}\left(M^{\prime \prime}\right)=0$ for all generic $z_{v}$ by hypothesis and (5.10), $M^{\prime \prime}$ is a Buchsbaum $R$-module. Moreover it follows from the latter half of (5.9) that

$$
\begin{gathered}
\sum_{j=0}^{v-2}\left(\begin{array}{c}
v-2 \\
j
\end{array}\right) h_{\mathfrak{m}}^{j}\left(M^{\prime}\right)=\sum_{j=0}^{v-2}\left(\begin{array}{c}
v-2 \\
j
\end{array}\right)\left\{h_{\mathfrak{m}}^{j}(M)+h_{\mathfrak{m}}^{j+1}(M)\right\} \\
=\sum_{j=0}^{v-1}\left(\begin{array}{c}
v-1 \\
j
\end{array}\right) h_{\mathfrak{m}}^{j}(M)=h_{\mathfrak{m}}^{0}\left(M^{\prime \prime}\right) .
\end{gathered}
$$

We find therefore with the use of elementary properties of multiplicity that

$$
\begin{aligned}
& l_{R}\left(M^{\prime} /\left(z_{1}, \ldots, z_{v-1}\right) M^{\prime}\right)=l_{R}\left(M^{\prime \prime} / g M^{\prime \prime}\right) \\
& \quad=e\left((g) ; M^{\prime \prime}\right)+h_{\mathrm{m}}^{0}\left(M^{\prime \prime}\right)=\operatorname{deg}\left(M^{\prime}\right)+\sum_{j=0}^{v-2}\left(\begin{array}{c}
v-2 \\
j
\end{array}\right) h_{\mathrm{m}}^{j}\left(M^{\prime}\right)
\end{aligned}
$$

for all $\left(z_{1}, \ldots, z_{v-1}\right)$ contained in a certain Zariski open subset of $R_{1}^{\oplus(v-1)}$. Hence by the induction hypothesis $M^{\prime}$ is a Buchsbaum $R$-module. Now let $g_{1}, \ldots, g_{v}$ be an arbitrary homogeneous system of parameters for $M$. As we have just seen $M / g_{1} M$ is a Buchsbaum $R$-module and $0:_{M} g_{1}=0:_{M} \mathfrak{m}$. The sequence $g_{1}, \ldots, g_{v}$ is therefore a weak $M$-sequence, hence $M$ is Buchsbaum by [SV, Chapter I, Theorem 3.7].

Theorem (5.12). We have

$$
m_{r-p} \leq \sum_{j=0}^{p}\left(\begin{array}{l}
p \\
j
\end{array}\right) h_{\mathfrak{m}}^{j}(A)
$$

for all $p(c \leq p<d)$. Moreover the following three conditions are equivalent:

(1) $A$ is a Buchsbaum ring,

(2) equality holds in (5.12.1) for all $p \quad(c \leq p<d)$,

(3) $m_{r-d+1}=\sum_{j=0}^{d-1}\left(\begin{array}{c}d-1 \\ j\end{array}\right) h_{\mathrm{m}}^{j}(A)$,

where we understand $m_{r-d+1}=0$ when $c=d$.

Proof. Since the case $c=d$ is trivial, we will assume $0 \leq c<d$. It follows from (2) of (1.2) and (2.1) that $m_{r-p} \leq h_{\mathrm{m}}^{0}\left(A^{\langle p\rangle}\right)$ for $c \leq p<d$, which together 
with (5.9) yields (5.12.1). To prove the equivalence of (1), (2), (3), note first that

$$
m_{r-d+1}=l_{R}\left(A^{\langle d\rangle}\right)-\operatorname{deg}(A) .
$$

In fact

$$
l_{R}\left(A^{\langle d\rangle}\right)=\operatorname{deg}\left(A^{\langle d\rangle}\right)=(-1)^{r-d} \sum_{i=1}^{r-d}(-1)^{i} \sum_{l=1}^{m_{i}}\left(\begin{array}{c}
n_{l}^{i} \\
r-d+1-i
\end{array}\right)
$$

by (1.6) applied to $I+x(r-d) R / x(r-d) R \hookrightarrow R / x(r-d) R=k\left[x_{1}, \ldots, x_{r-d}\right]$, therefore $\operatorname{deg}(A)=l_{R}\left(A^{\langle d\rangle}\right)-m_{r-d+1}$ again by (1.6). Now the implication (1) $\Rightarrow(2)$ follows from (1) of $(5.4),(2) \Rightarrow(3)$ is trivial and (3) $\Rightarrow$ (1) follows from $(5.11)$, since $(5.12 .2)$ holds for every generic $r$-tuple $x=\left(x_{1}, \ldots, x_{r}\right)$ of linear forms of $R$.

\section{AN EXISTENCE THEOREM}

Let $c, d, r$ be nonnegative integers satisfying $c \leq d<r, d \geq 1$. We denote by $\mathscr{B}_{B}(c, d, r)$ the set of sequences of integers larger than one, of the form $\left(n^{i}\right)_{1 \leq i \leq r-c}=\left(n^{1} ; \ldots ; n^{i} ; \ldots ; n^{r-c}\right)$ with nondecreasing sequences $n^{i}=$ $\left(n_{1}^{i}, \ldots, n_{m_{i}}^{i}\right)\left(m_{i} \geq 1,1 \leq i \leq r-c\right)$, which fulfill the following conditions.

(6.1) There are monomials $g_{l}^{i} \in k\left[y_{1}, \ldots, y_{i}\right] y_{i} \quad\left(1 \leq i \leq r-d, 1 \leq l \leq m_{i}\right)$ different from zero such that $\operatorname{deg}\left(g_{l}^{i}\right)=n_{l}^{i}$ for all $i, l, g_{l}^{i} k[y(i-1)] \cap$ $g_{l^{\prime}}^{i^{\prime}} k\left[y\left(i^{\prime}-1\right)\right]=0$ for $(i, l) \neq\left(i^{\prime}, l^{\prime}\right)$ and the set

$$
J:=\bigoplus_{i=1}^{r-d} \bigoplus_{l=1}^{m_{i}} g_{l}^{i} k[y(i-1)]
$$

is a Borel fixed ideal in $R$ with $\operatorname{dim}(R / J)=d$, where $y=\left(y_{1}, \ldots, y_{r}\right)$ is an $r$-tuple of indeterminates over $k$ and $R=k\left[y_{1}, \ldots, y_{r}\right]$.

(6.2) There are sequences of integers $w^{j}=\left(w_{1}^{j}, \ldots, w_{v_{j}}^{j}\right)(c \leq j<d)$ which have the properties (1) and (2) of (5.4) with respect to $n^{r-p} \quad(c \leq p \leq$ d).

Likewise we denote by $\mathscr{H}_{B}(c, d, r)$ the set of nontrivial homogeneous ideals in $R$ such that the factor rings defined by them are Buchsbaum of depth $c$ and dimension $d$. Recall that by definition an ideal $J$ generated by monomials in $y_{1}, \ldots, y_{r}$ is Borel fixed if and only if $\prod_{i=1}^{r}\left(\sum_{j=1}^{i-1} a_{i j} y_{j}+y_{i}\right)^{\mu_{i}} \in J$ for all $\prod_{i=1}^{r} y_{i}^{\mu_{i}} \in J, a_{i j} \in k \quad(1 \leq j<i \leq r)$. Since $B(I) \in \mathscr{B}_{B}(c, d, r)$ for every $I \in \mathscr{H}_{B}(c, d, r)$ by (1.1) and (5.4), the assignment $I \mapsto B(I)$ induces a map 
from $\mathscr{H}_{B}(c, d, r)$ to $\mathscr{B}_{B}(c, d, r)$. The purpose of this section is to prove that this map is surjective at least in the case $\operatorname{char}(k)=0$.

The notation being as above, we will assume for a while that

$$
g_{l}^{r-d} y_{p} / y_{r-d} \in J \text { for all } l, p\left(1 \leq l \leq m_{r-d}, 1 \leq p \leq r-d\right)
$$

instead of the Borel fixedness of $J$. This condition always holds when $\operatorname{char}(k)$ $=0$ and $J$ is Borel fixed. Put $S=k[y(r-d)], \mathfrak{n}=y(r-d) S \subset S$ and let $\left(K_{\bullet}(S), \partial_{\bullet}\right)$ denote the Koszul complex of $S$ with respect to $y(r-d)=$ $\left(y_{r-d+1}, \ldots, y_{r}\right)$. First we find by the proof of $(1.3)$ that $R / J$ is a finitely generated graded free $S$-module with basis $\left\{y^{\nu} \mid y^{\nu} \in k\left[y_{1}, \ldots, y_{r-d}\right] \backslash J\right\}$, in particular $R / J$ is Cohen-Macaulay. On the other hand, since $\left(\left(w^{j}+j+1\right)^{\left(\begin{array}{c}d \\ j\end{array}\right)}\right)_{c \leq j<d}$ is a subsequence of $n^{r-d}$ up to permutation by hypothesis, there is an injective map $l:\left\{(j, l, t) \mid c \leq j<d, 1 \leq l \leq v_{j}, 1 \leq t \leq\left(\begin{array}{c}d \\ j\end{array}\right)\right\} \rightarrow\left\{l \mid 1 \leq l \leq m_{r-d}\right\}$ such that $n_{l(j, l, t)}^{r-d}=w_{l}^{j}+j+1$ for all $j, l, t$. Put $g_{j, l, t}^{\prime}=g_{l(j, l, t)}^{r-d} / y_{r-d}$. Then each monomial $g_{j, l, t}^{\prime}$ is a member of the free basis of $R / J$ over $S$ as described above and $\operatorname{deg}\left(g_{j, l, t}^{\prime}\right)=w_{l}^{j}+j$. Moreover since $\operatorname{rank}_{S}\left(K_{j}(S)\right)=\left(\begin{array}{l}d \\ j\end{array}\right)$, we may regard $\bigoplus_{t=1}^{\left(\begin{array}{c}d \\ j\end{array}\right)} g_{j, l, t}^{\prime} S \hookrightarrow R / J$ as the graded $S$-module $K_{j}(S)\left(-w_{l}^{j}\right)$ for all $j, l$ $\left(c \leq j<d, 1 \leq l \leq v_{j}\right)$. Let $f_{j, l, u}(1 \leq u \leq(\underset{j+1}{d}))$ be the homogeneous polynomials contained in $\sum_{t=1}^{\left(\begin{array}{c}d \\ j\end{array}\right)} g_{j, l, t}^{\prime} \mathfrak{n}$ which correspond to the generators of $\operatorname{Im}\left(\partial_{j+1}\right) \subset K_{j}(S)$ through this identification for each $j, l$ and let $I$ denote the ideal in $R$ generated by $\left\{f_{j, l, u} \mid c \leq j<d, 1 \leq l \leq v_{j}, 1 \leq u \leq\left(\begin{array}{c}d \\ j+1\end{array}\right)\right\}$ and $J$. Put $A=R / I$.

Proposition (6.4). Assume (6.3). Then with the notation above $A$ is a graded Buchsbaum ring such that $H_{\mathrm{m}}^{j}(A)=0$ for $0 \leq j<c, H_{\mathrm{m}}^{j}(A) \cong k\left(-w^{j}\right)$ for $c \leq j<d, \operatorname{depth}_{\mathrm{m}}(A)=c$ and $\operatorname{dim}(A)=d$.

Proof. It follows from (6.3) that the graded $S$-module $A$ is isomorphic to the direct sum of $\bigoplus_{j=c}^{d-1} \bigoplus_{l=1}^{v_{j}} K_{j}(S)\left(-w_{l}^{j}\right) / \operatorname{Im}\left(\partial_{j+1}\right)$ and a suitable free $S$-module $C$. Since $\operatorname{deg}\left(g_{l}^{r-d}\right) \geq 2$ for all $l\left(1 \leq l \leq m_{r-d}\right)$ by hypothesis, this $C$ contains $S$ as a direct summand by the construction of $I$. Hence $\operatorname{dim}(A)=d$, $H_{\mathrm{m}}^{j}(A) \cong H_{\mathrm{n}}^{j}(A) \cong(S / \mathfrak{n})\left(-w^{j}\right)$ for $c \leq j<d, H_{\mathrm{m}}^{j}(A)=0$ for $0 \leq j<c$ and

$$
\begin{aligned}
\operatorname{deg}(A) & =\operatorname{deg}(R / J)-\sum_{j=c}^{d-1} \operatorname{deg}\left(\operatorname{Im}\left(\partial_{j+1}\right)\right) v_{j} \\
& =l_{R}\left(R / J+\left(z_{1}, \ldots, z_{d}\right) R\right)-\sum_{j=0}^{d-1}\left(\begin{array}{c}
d-1 \\
j
\end{array}\right) h_{\mathrm{m}}^{j}(A) .
\end{aligned}
$$

Put $z_{i}=y_{r-d+i}-\sum_{p=1}^{r-d} b_{i p} y_{p}$ for $1 \leq i \leq d$ with arbitrary $b_{i p} \in k$. Since $f_{j, l, u} \in \sum_{t} g_{j, l, t}^{\prime} \mathfrak{n}$ and $g_{j, l, l}^{\prime} y_{p} \in J$ for $1 \leq p \leq r-d$ by (6.3), we find 
$A /\left(z_{1}, \ldots, z_{d}\right) A=R / J+\left(z_{1}, \ldots, z_{d}\right) R$. Moreover the $d$-tuples of the linear forms $z_{1}, \ldots, z_{d}$ defined as above constitute a certain Zariski open subset of the grassmannian of $d$-dimensional $k$-vector subspaces of $R_{1}$. Therefore $A$ satisfies the hypothesis of $(5.11)$ by $(6.4 .1)$ and hence is a Buchsbaum ring. To verify $\operatorname{depth}_{\mathrm{m}}(A)=c$ it is enough to notice that $v_{c} \geq 1$ by $(6.2)$ when $c<d$.

For the computation of $B(I)$, we need the following

Lemma (6.5). Let $J \subset R$ be a Borel fixed ideal generated by monomials in $y_{1}, \ldots, y_{r}$ and let $\Upsilon$ be an invertible matrix of size $r$ with components in $k$ satisfying $\operatorname{det}\left(\Upsilon_{\left\langle i_{1}, \ldots, i_{p}\right\rangle}\right) \neq 0$ for all $1 \leq i_{1}<\cdots<i_{p} \leq r \quad(1 \leq p \leq r)$, where $\Upsilon_{\left\langle i_{1}, \ldots, i_{p}\right\rangle}$ denotes the square matrix of size $p$ whose $\left(q, q^{\prime}\right)$-component is the $\left(i_{q}, i_{q^{\prime}}\right)$-component of $\Upsilon$. Then for each monomial $\prod_{i=1}^{r} y_{i}^{\mu_{i}} \in J$ there is a homogeneous polynomial $f \in J$ such that in $(x ; f)=\prod_{i=1}^{r} x_{i}^{\mu_{i}}$ with respect to $x=\left(x_{1}, \ldots, x_{r}\right):=\left(y_{1}, \ldots, y_{r}\right)^{t} \Upsilon^{-1}$.

Proof. By elementary linear algebra there are a lower triangular invertible matrix $\Upsilon^{\prime}$ and an upper triangular invertible $\Upsilon^{\prime \prime}=\left(v_{i j}^{\prime \prime}\right)$ satisfying $\Upsilon=\Upsilon^{\prime} \Upsilon^{\prime \prime}$, so that $\left(x_{1}, \ldots, x_{r}\right)^{t} \Upsilon^{\prime \prime}=\left(y_{1}, \ldots, y_{r}\right)^{t} \Upsilon^{\prime-1}$. It follows therefore from the Borel fixedness that $f:=\prod_{i=1}^{r}\left(\sum_{j=i}^{r} v_{i j}^{\prime \prime} x_{j}\right)^{\mu_{i}} / \prod_{i=1}^{r} v_{i i}^{\prime \prime} \in J$ for each monomial $\prod_{i=1}^{r} y_{i}^{\mu_{i}}$ $\in J$. Clearly $\operatorname{in}(x ; f)=\prod_{i=1}^{r} x_{i}^{\mu_{i}}$.

Proposition (6.6). Let the notation be as before and suppose that the ideal $J$ defined in (6.1) is Borel fixed and satisfies (6.3) as well. Then the basic sequence of the ideal I given by the above method coincides with $\left(n^{i}\right)_{1 \leq i \leq r-c}$.

Proof. Let $x=\left(x_{1}, \ldots, x_{r}\right)$ be a generic $r$-tuple of linear forms of $R$ and let $g_{l}^{i}(x)$ denote the monomial in $x$ obtained from $g_{l}^{i}$ by substituting $x$ for $y$ for each $i, l$. By (6.5) there are homogeneous $f_{l}^{i} \in J\left(1 \leq i \leq r-d, 1 \leq l \leq m_{i}\right)$ such that in $\left(x ; f_{l}^{i}\right)=g_{l}^{i}(x)$. Let $f_{l}^{i\langle p\rangle}$ denote $f_{l}^{i}\left(x_{1}, \ldots, x_{r-p}, 0, \ldots, 0\right)$ for $0 \leq p \leq r$. Since $\operatorname{in}\left(x ; f_{l}^{i}\right) k[x(i-1)] \cap \operatorname{in}\left(x ; f_{l^{\prime}}^{i^{\prime}}\right) k\left[x\left(i^{\prime}-1\right)\right]=0$ for $(i, l) \neq$ $\left(i^{\prime}, l^{\prime}\right)$ by $(6.1)$, it follows from (4.3) that the sum

$$
J^{\prime}:=\sum_{i=1}^{r-d} \sum_{l=1}^{m_{i}} f_{l}^{i} k[x(i-1)] \subset J
$$

is direct and therefore $\operatorname{dim}_{k}\left(J^{\prime}\right)_{t}=\operatorname{dim}_{k}(J)_{t}$ for all $t \geq 0$. Hence $J^{\prime}=J$. Moreover since $f_{l}^{i\langle p\rangle}=0$ for all $i, l, p \quad\left(0 \leq r-p<i \leq r-d, 1 \leq l \leq m_{i}\right)$, we have

$$
J+x(r-p) R / x(r-p) R=\bigoplus_{i=1}^{r-p} \bigoplus_{l=1}^{m_{i}} f_{l}^{i\langle p\rangle} k\left[x_{i}, \ldots, x_{r-p}\right]
$$

for $d \leq p \leq r-1$. Let $\left(n^{\prime i}\right)_{1 \leq i \leq r-c^{\prime}}$ be the basic sequence of $I$. First $x(r-d) R=\left(z_{1}, \ldots, z_{d}\right) R$ with suitable linear forms $z_{1}, \ldots, z_{d}$ defined as in the proof of (6.4), so that $I+x(r-p) R / x(r-p) R=J+x(r-p) R / x(r-p) R$ for 
$d \leq p \leq r-1$ by (6.3). Computing $\operatorname{dim}_{k}(J+x(r-p) R / x(r-p) R)_{t}$ with the use of (6.6.1), we find therefore by the observation preceding (1.5) that $n^{\prime i}=n^{i}$ for $1 \leq i \leq r-d$. Secondly since $A$ is Buchsbaum with $\operatorname{depth}_{\mathrm{m}}(A)=c$, $\operatorname{dim}(A)=d$ by (6.4), it follows from (5.4), (6.2) and (2) of (1.3) that $c^{\prime}=c$, $n^{\prime i}=n^{i}$ for $r-d+1 \leq i \leq r-c$. Thus $B(I)=\left(n^{i}\right)_{1 \leq i \leq r-c}$.

Corollary (6.7). Let $c, d, r$ be nonnegative integers satisfying $c \leq d<r, d \geq 1$ and suppose $\operatorname{char}(k)=0$. Then for every $\left(n^{i}\right)_{1 \leq i \leq r-c} \in \mathscr{B}_{B}(c, d, r)$ there exists a homogeneous ideal $I \in \mathscr{H}_{B}(c, d, r)$ such that $B(I)=\left(n^{i}\right)_{1 \leq i \leq r-c}$.

Corollary (6.8). The lower bound estimate described in (2) of (5.6) is sharp when $c<d$.

Proof. Suppose $d=r-2 \geq 1, c<d$. Given nonnegative integers $v_{j} \quad(c<$ $j<d)$ and a positive $v_{c}$ with $n:=\sum_{j=c}^{d-1}\left(\begin{array}{l}d \\ j\end{array}\right) v_{j} \geq 2$, we put $m_{1}=1, m_{r-p}=$ $\sum_{j=c}^{\min (p, d-1)}\left(\begin{array}{l}p \\ j\end{array}\right) v_{j}$ for $c \leq p \leq d, n_{l}^{i}=n$ for all $i, l \quad(1 \leq i \leq r-c, 1 \leq$ $\left.l \leq m_{i}\right), w^{j}=(n-j-1)^{n_{j}}$ for $c \leq j<d$ and $g_{1}^{1}=y_{1}^{n}, g_{l}^{2}=y_{1}^{n-l} y_{2}^{l}$ for $1 \leq l \leq m_{2}=n$. Apply (6.4) and (6.6).

Remark (6.9). (1) We gave a proof of (6.7) for the case $c=1, d=2, r=4$ by another method in [A2, Lemma 4.2]. In this case we know more. According to [BM3, §4] every element of $\mathscr{B}_{B}(1,2,4)$ is realized by the ideal defining a configuration of lines in $\mathbf{P}^{3}$ which is arithmetically Buchsbaum.

(2) The choice of the monomials $g_{l}^{i}$ is not unique. If even though $g_{l}^{i}$ 's do not fulfill (6.3) the Borel fixedness of $J$ implies the existence of other monomials $g_{l}^{\prime i}\left(1 \leq i \leq r-d, 1 \leq l \leq m_{i}\right)$ satisfying $(6.3)$ as well as the conditions in (6.1), then (6.7) holds without the assumption $\operatorname{char}(k)=0$. But we have not yet succeeded in proving such implication except for the simple cases $r-d=1,2,3$.

\section{Graded Buchsbaum integral domains of Codimension two}

We will give a characterization of the elements of $\mathscr{B}_{B}(c, d, r)$ corresponding to graded Buchsbaum integral domains in the case $d=r-2 \geq 2$, $\operatorname{char}(k)=0$.

Let $\left(n^{i}\right)_{1<i<r-c}$ be an element of $\mathscr{B}_{B}(c, r-2, r)$. Then $m_{1}=1, m_{2}=$ $n_{1}^{1} \leq n_{1}^{2} \leq n_{2}^{2} \leq \cdots \leq n_{m_{2}}^{2}$ and there are nondecreasing sequences $w=$ $\left(w_{1}, \ldots, w_{v}\right), w^{j}=\left(w_{1}^{j}, \ldots, w_{v_{j}}^{j}\right) \quad(c \leq j<r-2)$ such that

$$
n^{2}=\left(\left(\left(w^{j}+j+1\right)^{\left(\begin{array}{c}
r-2 \\
j
\end{array}\right)}\right)_{c \leq j<r-2}, w\right), \quad n^{r-p}=\left(\left(w^{j}+j+1\right)^{\left(\begin{array}{c}
p \\
j
\end{array}\right)}\right)_{c \leq j \leq p}
$$

for $c \leq p<r-2$ up to permutation respectively. Note that they are determined uniquely by this condition. We begin by proving the existence of what M. Chang calls $\Omega$-resolutions (see [C, §0]) in such a way that the connection with standard free resolutions becomes clear. Fix $\left(n^{i}\right)_{1 \leq i \leq r-c} \in \mathscr{B}_{B}(c, r-2, r)(r \geq 2), w, w^{j}$ $(c \leq j<r-2)$. 
Proposition (7.1). Let $I$ be a homogeneous ideal in $R$ belonging to $\mathscr{H}_{B}(c, r-2, r)$ with $B(I)=\left(n^{i}\right)_{1 \leq i \leq r-c}$. Then we have an exact sequence of the form

$$
\begin{aligned}
0 \rightarrow R(-w-1) \oplus & \left\{\bigoplus_{j=c}^{r-3} R\left(-w^{j}-j-1\right)^{\left.\left(\begin{array}{r}
r-1 \\
j
\end{array}\right)\right\}}\right. \\
& \rightarrow R\left(-n_{1}^{1}\right) \oplus R(-w) \oplus\left\{\bigoplus_{j=c}^{r-3} \operatorname{Syz}_{j+1}^{R}(k)\left(-w^{j}\right)\right\} \rightarrow I \rightarrow 0 .
\end{aligned}
$$

Proof. First of all $H_{\mathrm{m}}^{j}(R / I)=0$ for $0 \leq j<c, H_{\mathrm{m}}^{j}(R / I) \cong k\left(-w^{j}\right)$ for $c \leq j<d$ by (1) of (5.4) and the uniqueness of $w^{j}$ 's. Let $x=\left(x_{1}, \ldots, x_{r}\right)$ be a generic $r$-tuple of linear forms of $R, f_{1}^{1}$ the polynomial in $I$ described in (1.1) $(i=l=1), S=k[x(1)], J=f_{1}^{1} R$ and $N=\bigoplus_{t=0}^{n_{1}^{1}-1} x_{1}^{t} S \subset R$. Then $I / J$ has the direct sum decomposition (5.4.4) as a $k$-vector space. Denote the homogeneous polynomials in $I \cap N \cong I / J$ corresponding to the generators of the direct summands of the form $k[x(r-p-1)]\left(-w^{j}-j-1\right)^{\left(\begin{array}{c}p \\ j\end{array}\right)}$ on the righthand side of (5.4.4) by $g_{l}^{r-p} \quad\left(c \leq p \leq r-3,1 \leq l \leq m_{r-p}\right.$ or $p=r-2, v+1 \leq$ $\left.l \leq m_{2}\right)$. Since (5.4.4) is obtained from (5.3) and (5.4.3), we have

$$
\sum_{l=l+1}^{m_{2}} g_{l}^{2} S+\sum_{i=3}^{r-c} \sum_{l=1}^{m_{i}} g_{l}^{i} S \cong \bigoplus_{j=c}^{r-3} \operatorname{Syz}_{j+1}^{S}(k)\left(-w^{j}\right) .
$$

Put $g_{1}^{1}=f_{1}^{1}$ and let $g_{l}^{2}(1 \leq l \leq v)$ be the homogeneous polynomials in $I \cap N$ corresponding to the generators of $S(-w)$. Obviously

$$
I=\bigoplus_{i=1}^{r-c} \bigoplus_{l=1}^{m_{i}} g_{l}^{i} k[x(i-1)]
$$

We will look carefully into the standard free resolution $\Psi_{\bullet}: L_{\bullet} \rightarrow I$ of $I$ starting from this basis. With the notation of section three put

$$
\begin{aligned}
& \tilde{L}_{0}=\left(\bigoplus_{l=l+1}^{m_{2}} Y_{l}^{2} S\right) \oplus\left(\bigoplus_{i=3}^{r-c} \bigoplus_{l=1}^{m_{i}} Y_{l}^{i} S\right), \\
& \tilde{L}_{p}=\bigoplus_{(\alpha, i) \in \varepsilon(p ; 2, r-c)} \bigoplus_{l=1}^{m_{i}}\left(X_{c} \otimes Y_{l}^{i}\right) S \quad \text { for } 1 \leq p \leq r-c-2 .
\end{aligned}
$$

Claim (7.1.2). $\Psi_{\beta_{1}}^{1, j}$ and the upper $v$ rows of $\Psi_{\beta_{1}}^{2, j}$ are zero for all $\beta_{1}, j \quad(2 \leq$ $\left.\beta_{1}<j \leq r-c\right)$.

Claim (7.1.3). The maps $\left.\Psi_{p}\right|_{\check{L}_{p}}(0 \leq p \leq r-c-2)$ yield the minimal free resolution of $\bigoplus_{j=c}^{r-3} \mathrm{Syz}_{j+1}^{S}(k)\left(-w^{j}\right)$ over $S$ given by Koszul complexes.

The first claim follows immediately from (7.1.1), and so does the second since the standard free resolution of $\mathrm{Syz}_{j+1}^{S}(k)$ coincides with $\partial_{\bullet+j+1}: K_{\bullet+j+1}(S) \rightarrow$ $\mathrm{Syz}_{j+1}^{S}(k)$ by (3.12). 
Consequently $\Psi_{\beta_{1}}^{i, j} \in \operatorname{MAT}\left(x\left(\beta_{1}\right)\right)$ for $1 \leq i \leq \beta_{1}<j \leq r-c, \beta_{1} \geq 2$. On the other hand since $g_{1}^{1}-\operatorname{in}\left(x ; g_{1}^{1}\right)$ and $g_{l}^{i}$ 's $(i \geq 2)$ all lie in $N$, we find $\Psi_{1}^{1, j} \in \operatorname{MAT}(x(1))$ for $1<j \leq r-c$. The map $\Psi_{1}$ therefore satisfies (3.5.1). Set

$$
\begin{aligned}
& L_{0}^{\prime}=Y_{1}^{1} R \oplus\left(\bigoplus_{l=1}^{\prime} Y_{l}^{2} R\right), \quad L_{1}^{\prime}=\bigoplus_{l=1}^{v}\left(X_{1} \otimes Y_{l}^{2}\right) R, \\
& L_{p}^{\prime \prime}=\tilde{L}_{p} \otimes_{S} R \text { for } 0 \leq p \leq r-c-2, \\
& L_{1}^{\prime \prime \prime}=\left\{\bigoplus_{l=v+1}^{m_{2}}\left(X_{1} \otimes Y_{l}^{2}\right) R\right\} \oplus\left\{\bigoplus_{i=3}^{r-c} \bigoplus_{l=1}^{m_{i}}\left(X_{1} \otimes Y_{l}^{i}\right) R\right\} \oplus L_{1}^{\prime \prime} .
\end{aligned}
$$

Then $L_{0}=L_{0}^{\prime} \oplus L_{0}^{\prime \prime}, L_{1}=L_{1}^{\prime} \oplus L_{1}^{\prime \prime \prime}$. Let $\Psi_{2}^{\prime}, \Psi_{2}^{\prime \prime \prime}$ denote the composite maps of $\Psi_{1}$ and the projections to $L_{1}^{\prime}, L_{1}^{\prime \prime \prime}$ respectively. By $(3.5), \Psi_{1,\left(\beta_{1}, \beta_{2}\right)}^{2, j}=0$ for $\beta_{1}>1$ and $\Psi_{1,\left(1, \beta_{2}\right)}^{2, j}=-\Psi_{\beta_{2}}^{2, j}$ for $\beta_{1}=1, \beta_{2}>1$, hence $\Psi_{2}^{\prime}=0$ by (7.1.2).

Put $C_{p}=\bigoplus_{j=c}^{r-3} K_{p+j+1}(R)\left(-w^{j}\right), \theta_{p}=\bigoplus_{j=c}^{r-3}\left(\partial_{p+j+1}^{\oplus v_{j}}\right)$ for $p=0,1,2$. The module $\operatorname{Im}\left(\Psi_{1}\right)=L_{1}^{\prime} \oplus \operatorname{Coker}\left(\Psi_{2}^{\prime \prime \prime}\right)$ is Buchsbaum by $(5.5)$ and $\operatorname{rank}_{R}\left(L_{1}^{\prime \prime \prime}\right)=$ $m_{2}-v+\sum_{i=3}^{r-c}(i-1) m_{i}=\sum_{j=c}^{r-3}\left(\begin{array}{c}r \\ j+2\end{array}\right) v_{j}$ by $(5.5 .1)$, so

$$
\operatorname{Coker}\left(\Psi_{2}^{\prime \prime \prime}\right) \cong \bigoplus_{j=c}^{r-3} \operatorname{Syz}_{j+2}^{R}(k)\left(-w^{j}\right)
$$

by (5.2). Besides there are isomorphisms $\rho_{1}: C_{1} \rightarrow L_{1}^{\prime \prime \prime}, \rho_{2}: C_{2} \rightarrow L_{2}$ such that $\Psi_{2}^{\prime \prime \prime} \circ \rho_{2}=\rho_{1} \circ \theta_{2}$. Look at the commutative diagram

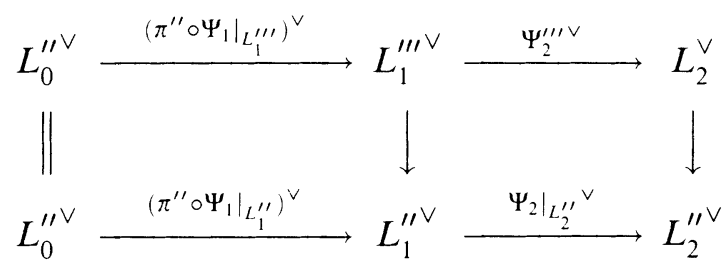

Here $\pi^{\prime \prime}: L_{0} \rightarrow L_{0}^{\prime \prime}$ and the vertical arrows are projections. Since the dual of a Koszul complex is again a Koszul complex, the second row is exact and the image of the free basis of $L_{0}^{\prime \prime \vee}$ through $\left(\left.\pi^{\prime \prime} \circ \Psi_{1}\right|_{L_{1}^{\prime \prime}}\right)^{\vee}$ yields a system of minimal generators of $\operatorname{Ker}\left(\left.\Psi_{2}\right|_{L_{2}^{\prime \prime}}{ }^{\vee}\right)$ by (7.1.3). The first row can therefore be completed to an exact sequence $D^{\vee} \stackrel{\Psi^{\vee}}{\longrightarrow} L_{1}^{\prime \prime \prime} \vee L_{2}^{\vee}$ such that $D$ is free, $L_{0}^{\prime \prime}$ is a direct summand of $D,\left.\Psi^{\vee}\right|_{L_{0}^{\prime \prime} \vee}=\left(\left.\pi^{\prime \prime} \circ \Psi_{1}\right|_{L_{1}^{\prime \prime \prime}}\right)^{\vee}$ and $\Psi^{\vee} \equiv 0(\bmod \mathfrak{m})$. Since $C_{0}^{\vee} \stackrel{\theta_{1}^{\vee}}{\longrightarrow} C_{1}^{\vee} \stackrel{\theta_{2}^{\vee}}{\longrightarrow} C_{2}^{\vee}$ is exact and $\theta_{1}^{\vee} \equiv 0(\bmod \mathfrak{m})$, there is an isomorphism $\rho_{0}: C_{0} \rightarrow D$ such that $\theta_{1}^{\vee} \circ \rho_{0}^{\vee}=\rho_{1}^{\vee} \circ \Psi^{\vee}$. Let $\zeta^{\prime \prime}$ denote the map $\rho_{0}$ followed by the projection to $L_{0}^{\prime \prime}$ and put $C_{0}^{\prime \prime}=\rho_{0}^{-1}\left(L_{0}^{\prime \prime}\right)$. Then $\zeta^{\prime \prime} \circ \theta_{1}=\pi^{\prime \prime} \circ \Psi_{1} \circ \rho_{1}$ 
and $\left.\zeta^{\prime \prime}\right|_{C_{0}^{\prime \prime}}$ is an isomorphism. Likewise taking duals, we can construct a map $\zeta^{\prime}: C_{0} \rightarrow L_{0}^{\prime}$ such that $\zeta^{\prime} \circ \theta_{1}=\pi^{\prime} \circ \Psi_{1} \circ \rho_{1}$, where $\pi^{\prime}$ is the projection $L_{0} \rightarrow L_{0}^{\prime}$.

Set

$$
\zeta=\left(\begin{array}{cc}
\mathrm{id}_{L_{0}^{\prime}} & 0 \\
\zeta^{\prime} & \zeta^{\prime \prime}
\end{array}\right): L_{0}^{\prime} \oplus C_{0} \rightarrow L_{0}^{\prime} \oplus L_{0}^{\prime \prime}=L_{0} .
$$

Let us consider the commutative diagram

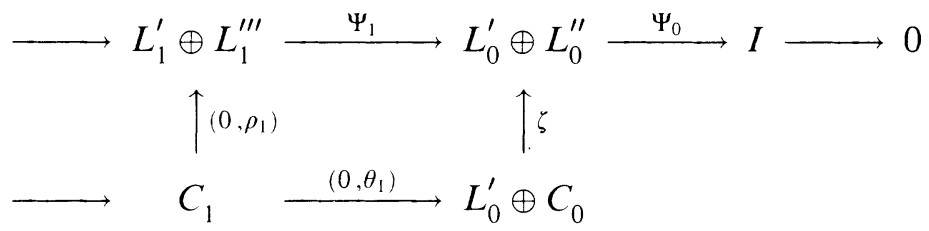

Since $\left.\zeta\right|_{L_{0}^{\prime} \oplus C_{0}^{\prime \prime}}$ is an isomorphism and $L_{1}^{\prime}, C_{0} / C_{0}^{\prime \prime}$ are free, there are maps $\chi^{\prime}: L_{1}^{\prime} \rightarrow L_{0}^{\prime} \oplus C_{0}, \chi^{\prime \prime}: C_{0} \rightarrow L_{0}^{\prime} \oplus C_{0}$ such that $\zeta \circ \chi^{\prime}=\left.\Psi_{1}\right|_{L_{1}^{\prime}}, \operatorname{Im}\left(\chi^{\prime}\right) \subset$ $L_{0}^{\prime} \oplus C_{0}^{\prime \prime}, \zeta \circ \chi^{\prime \prime}=0,\left.\chi^{\prime \prime}\right|_{C_{0}^{\prime \prime}}=0, \operatorname{Im}\left(\chi^{\prime \prime}-\left(0, \mathrm{id}_{C_{0}}\right)\right) \subset L_{0}^{\prime} \oplus C_{0}^{\prime \prime}$. We can therefore construct a complex

$$
0 \rightarrow L_{1}^{\prime} \oplus\left(C_{0} / C_{0}^{\prime \prime}\right) \stackrel{\chi_{2}}{\longrightarrow} L_{0}^{\prime} \oplus\left(C_{0} / \theta_{1}\left(C_{1}\right)\right) \stackrel{\chi_{1}}{\longrightarrow} I \rightarrow 0,
$$

defining $\chi_{1}$ by $\Psi_{0} \circ \zeta$ and $\chi_{2}$ by $\chi^{\prime}, \chi^{\prime \prime}$. Observe $L_{0}^{\prime} \cong R\left(-n_{1}^{1}\right) \oplus R(-w)$, $L_{1}^{\prime} \cong R(-w-1), C_{0}^{\prime \prime} \cong \bigoplus_{j=c}^{r-3} K_{j+1}(S)\left(-w^{j}\right) \otimes_{S} R$ by (7.1.1) and the definition of $\tilde{L}_{0}, C_{0} / C_{0}^{\prime \prime} \cong \bigoplus_{j=c}^{r-3} R\left(-w^{j}-j-1\right)^{\left(\begin{array}{c}r-1 \\ j\end{array}\right)}, C_{0} / \theta_{1}\left(C_{1}\right) \cong \bigoplus_{j=c}^{r-3} \operatorname{Syz}_{j+1}^{R}(k)\left(-w^{j}\right)$. Now the remaining thing is to verify the exactness. Clearly $\chi_{1}$ is surjective. The exactness in the middle can be proved by diagram chasing. Finally comparing ranks, we find that $\chi_{2}$ is injective. Q.E.D.

Remark (7.2). (1) Another proof was given for the case $c=1,2, r=4$ in [A2, §3], [A 5, §2].

(2) Chang proved an analogue of Goto's structure theorem in the case of vector bundles on projective spaces. Applying it to the vector bundle $\mathscr{F}$ defined as an extension of the form $0 \rightarrow \bigoplus \mathscr{O}_{\mathbf{P}^{r-1}}\left(-t_{l}\right) \rightarrow \mathscr{F} \rightarrow \mathscr{I} \rightarrow 0$, she obtained (7.1) in the sheafified form except for the explicit expression of the twisting degrees in terms of the basic sequence. See $[C, \S \S 0,1]$ for the details.

Lemma (7.3). Let $I \subset R$ be a homogeneous ideal such that there is an exact sequence as stated in (7.1) for $I,\left(n^{i}\right)_{1 \leq i \leq r-c}$. Then $I \in \mathscr{H}_{B}(c, r-2, r)$ and $B(I)=\left(n^{i}\right)_{1 \leq i \leq r-c}$.

Proof. Set $A=R / I, B(I)=\left(n^{\prime i}\right)_{1 \leq i \leq r-c^{\prime}}$. The exact sequence which is assumed to exist allows us to compute the degree of the Hilbert polynomial of $A$. As a result we find $\operatorname{dim}(A)=r-2$. On the other hand, it follows from the proof of (5.2) that the canonical map $\operatorname{Ext}_{R}^{j}(k, A) \rightarrow H_{\mathrm{m}}^{j}(A)$ is surjective for all $j(0 \leq j<r-2)$ and that $H_{\mathrm{m}}^{j}(A) \cong 0$ for $0 \leq j<c, H_{\mathrm{m}}^{j}(A) \cong k\left(-w^{j}\right)$ for 
$c \leq j<r-2$. Therefore $A$ is Buchsbaum and moreover $c^{\prime}=c, n^{\prime i}=n^{i}$ for $3 \leq i \leq r-c$,

$$
n^{\prime 2}=\left(\left(\left(w^{j}+j+1\right)^{\left(\begin{array}{c}
r-2 \\
j
\end{array}\right)}\right)_{c \leq j<r-2}, w^{\prime}\right)
$$

up to permutation with a suitable nondecreasing sequence $w^{\prime}=\left(w_{1}^{\prime}, \ldots, w_{v^{\prime}}^{\prime}\right)$ by (5.4). Apply (7.1) to $I$. Then the assumed exact sequence and the exact sequence guaranteed by (7.1) yield two expressions of the function $\operatorname{dim}(I)_{t}$ $(t \geq 0)$ of the same form, with numerical data being possibly different from each other. Comparing them, we see

$$
\sum_{l=1}^{v^{\prime}}\left(\begin{array}{c}
t-w_{l}^{\prime}+r-2 \\
r-2
\end{array}\right)_{+}=\sum_{l=1}^{v}\left(\begin{array}{c}
t-w_{l}+r-2 \\
r-2
\end{array}\right)_{+} \quad \text { for all } t \geq 0
$$

since obviously $n_{1}^{\prime 1}=\min \left\{t \mid I_{t} \neq 0\right\}=n_{1}^{1}$. Hence $w^{\prime}=w, n^{\prime 2}=n^{2}$ and after all $B(I)=\left(n^{i}\right)_{1 \leq i \leq r-c}$.

We say that a sequence of integers $t_{1}, \ldots, t_{q}$ is connected if $t_{l} \leq t_{l+1} \leq t_{l}+1$ for all $l(1 \leq l \leq q-1)$ or $q=0$ (i.e. the sequence is empty). The following theorem is a generalization of [A5, Theorem].

Theorem (7.4). Assume that $k$ is algebraically closed. Let $u_{\min }$ and $u_{\max }$ denote the minimum and the maximum of $\left\{w_{l}^{j}+j+1 \mid c \leq j<r-2,1 \leq l \leq v_{j}\right\}$ respectively.

(1) If there exists a homogeneous prime ideal I belonging to $\mathscr{H}_{B}(c, r-2, r)$ such that $B(I)=\left(n^{i}\right)_{1 \leq i \leq r-c}$, then one of the following conditions holds.

$$
\begin{aligned}
& r \geq 4, c=r-2 \text { and the sequence } n^{2}=w \text { is connected, } \\
& r=4, c=1, n_{1}^{1}=2, v=0, v_{1}=1, w_{1}^{1} \geq 1, \\
& r \geq 4,0<c<r-2, n_{1}^{1} \geq 3, \\
& v=n_{1}^{1}-\sum_{j=c}^{r-3}\left(\begin{array}{c}
r-2 \\
j
\end{array}\right) v_{j} \geq u_{\max }-u_{\min }, \\
& w_{1} \leq u_{\min }, u_{\max }-1 \leq w_{v} \text { and the sequence } w \text { is connected } .
\end{aligned}
$$

(2) In the case $\operatorname{char}(k)=0$, these conditions are also sufficient for the existence of a prime ideal $I \in \mathscr{H}_{B}(c, r-2, r)$ with $B(I)=\left(n^{i}\right)_{1 \leq i \leq r-c}$.

Proof. It is enough to consider the problem of finding the conditions for the existence of integral arithmetically Buchsbaum subschemes of codimension two in $\mathbf{P}^{r-1}$ in terms of the basic sequences of their defining ideals under the assumption $r \geq 4,0<c \leq r-2$. This problem can be solved by entirely the same method as in [A5] and the answer is nothing but our assertion, so that we will explain only the necessary changes which has to be made in that argument.

Let $\mathscr{E}_{j}$ denote the vector bundle of rank $\left(\begin{array}{c}r-1 \\ j-1\end{array}\right)$ on $\mathbf{P}^{r-1}$ associated with the graded $R$-module $\operatorname{Syz}_{j}^{R}(k)(j)$ for each $j(2 \leq j \leq r)$. It follows from the property of the Koszul complex that $H^{0}\left(\mathscr{E}_{j}(t)\right)=0$ for $t<0, H^{j-1}\left(\mathscr{E}_{j}(-j)\right) \cong$ 
$k, H^{i}\left(\mathscr{E}_{j}(t)\right)=0$ for $0<i<r-1,(i, t) \neq(j-1,-j)$ and $\mathscr{E}_{j}$ is generated over $\mathscr{O}_{\mathbf{P}^{r-1}}$ by $H^{0}\left(\mathscr{E}_{j}\right) \cong\left[\mathrm{Syz}_{j}^{R}(k)\right]_{j}$. Note that $\mathscr{E}_{j}(-j)$ is isomorphic to the $(j-1)$ th exterior power of the cotangent bundle. Put

$$
\begin{aligned}
& \hat{u}_{\min }=\min \left(w_{1}, u_{\min }-1\right), \quad \hat{u}_{\max }=\max \left(w_{v}, u_{\max }-1\right), \\
& \hat{v}_{t}=\sharp\left\{l \mid w_{l}=t, 1 \leq l \leq v\right\}, \\
& v_{j, t}=\sharp\left\{l \mid w_{l}^{j}+j+1=t, 1 \leq l \leq v_{j}\right\}
\end{aligned}
$$

for $t \in \mathbf{Z}, c \leq j<r-2$, where $\hat{u}_{\min }=u_{\min }-1, \hat{u}_{\max }=u_{\max }-1$ if $v=0$ and $\hat{u}_{\min }=w_{1}, \hat{u}_{\max }=w_{v}$ if $c=r-2$. We set

$$
\mathscr{P}_{t}=\mathscr{O}_{\mathbf{P}^{r-1}}(-t)^{\hat{t}_{t}} \oplus\left\{\bigoplus_{j=c}^{r-3} \mathscr{E}_{j+1}(-t-1)^{v_{j, t+1}}\right\}
$$

for $t \in \mathbf{Z}$ and

$$
\begin{aligned}
& \mathscr{F}_{u}=\mathscr{O}_{\mathbf{P}^{r-1}}\left(-n_{1}^{1}\right) \oplus\left(\bigoplus_{t=\hat{u}_{\min }}^{u} \mathscr{P}_{t}\right), \quad \mathscr{G}_{u}=\bigoplus_{t=u+1}^{\hat{u}_{\max }} \mathscr{P}_{t}, \\
& \mathscr{L}_{u}=\bigoplus_{t=\hat{u}_{\min }}^{u} \mathscr{O}_{\mathbf{P}^{r-1}}(-t-1)^{\hat{v}_{t}+\sum_{j=c}^{r-3}\left(\begin{array}{c}
r-1 \\
j
\end{array}\right) v_{j, t+1}}
\end{aligned}
$$

for $\hat{u}_{\min } \leq u \leq \hat{u}_{\max }$. Then for each $I \in \mathscr{H}_{B}(c, r-2, r)$, the exact sequence in (7.1) yields a locally free resolution for the ideal sheaf $\mathscr{I}_{Z}$ of $Z:=\operatorname{Proj}(R / I)$ of the form

$$
0 \rightarrow \mathscr{L}_{\hat{u}_{\max }} \rightarrow \mathscr{F}_{\hat{u}_{\max }} \rightarrow \mathscr{I}_{Z} \rightarrow 0 .
$$

The integers $\hat{v}_{t}, \hat{u}_{\min }, \hat{u}_{\max }$ and the vector bundles $\mathscr{F}_{u}, \mathscr{G}_{u}, \mathscr{L}_{u}$ play the roles of $e_{n}, \alpha, \beta$ and $F_{m}, G_{m}, L_{m}$ defined in [A5, §2] respectively.

(1) The reasoning in the proof of [A5, Theorem (i)] applies to arbitrary integral arithmetically Buchsbaum subschemes of codimension two in $\mathbf{P}^{r-1}$ for all $r \geq 4$.

(2) Suppose that $\operatorname{char}(k)=0$ and that one of the three conditions holds. By the method described in the proof of [A5, Theorem (ii)], we can construct a closed subscheme $Z$ of codimension two in $\mathbf{P}^{r-1}$, which is smooth over $k$ in the outside of a closed subset of codimension larger than or equal to three in $Z$, such that $\mathscr{I}_{Z}$ has a locally free resolution of the form (7.4.4). For each point $o \in Z$, the local ring $\mathscr{O}_{Z, o}$ satisfies Serre's criterion of normality, since $\mathscr{O}_{Z, o}$ is Cohen-Macaulay by the existence of the resolution (7.4.4) and AuslanderBuchsbaum's theorem and since the singular locus of $Z$ is of codimension not less than two. Consequently $\mathscr{O}_{Z, o}$ is normal, in particular it is a local integral domain (cf. [M, Theorem 39]). On the other hand since $c \geq 2$ or $c=1$ and $w_{l}^{1} \geq 1$ for all $l\left(1 \leq l \leq v_{1}\right)$ if one of (7.4.1), (7.4.2), (7.4.3) holds, we find $H^{1}\left(\mathscr{I}_{Z}\right)=0$ by (7.4.4). The scheme $Z$ is therefore reduced, 
locally irreducible and connected, namely $Z$ is integral. Let $I$ denote the homogeneous ideal $\bigoplus_{t \geq 0} H^{0}\left(\mathscr{I}_{Z}(t)\right) \subset R$. Then the exact sequence of the global sections obtained from (7.4.4) coincides with the exact sequence stated in (7.1), so that $I \in \mathscr{H}_{B}(c, r-2, r)$ with $B(I)=\left(n^{i}\right)_{1 \leq i \leq r-c}$ by (7.3). Since $R / I$ is an integral domain as we have already seen, this completes the proof for the existence. Q.E.D.

Corollary (7.5). Let $I$ be a homogeneous prime ideal in $R$ such that $R / I$ is a graded Buchsbaum integral domain of depth $c<r-2$ and dimension $d=r-2$. Then

$$
\min \left\{t \mid I_{t} \neq 0\right\} \geq u_{\max }-u_{\min }+\sum_{j=c}^{r-3}\left(\begin{array}{c}
r-2 \\
j
\end{array}\right) h_{\mathrm{m}}^{j}(R / I),
$$

where $u_{\min }$ and $u_{\max }$ denote the minimal and the maximal elements of the set $\left\{t \mid h_{\mathrm{m}}^{j}(R / I)_{t-j-1} \neq 0\right.$ for some $\left.j \quad(c \leq j<r-2)\right\}$ respectively. Moreover this lower bound estimate for the minimal degree of generators is sharp when $\operatorname{char}(k)=0$.

Remark (7.6). (1) We do not know whether the assumption $\operatorname{char}(k)=0$ is indispensable or not for the proof of sufficiency.

(2) Following the same lines of argument, we can prove that every element of $\mathscr{B}_{B}(c, r-2, r) \quad(0<c \leq r-2, r \geq 3)$ is realized by an ideal defining reduced graded Buchsbaum ring if $\operatorname{char}(k)=0$ (cf. [C, §2]).

\section{AppendiX: Proof of Theorem (1.1)}

Let $y_{i}, z_{j}, \zeta_{i j}(1 \leq i \leq r, 1 \leq j \leq r)$ be indeterminates over $k$, $\zeta$ the square matrix of size $r$ whose $(i, j)$-component is $\zeta_{i j}, K:=k(\zeta)$ the rational function field generated over $k$ by all $\zeta_{i j}$ 's. We will fix a homogeneous ideal $I$ in $R=k\left[y_{1}, \ldots, y_{r}\right]$ different from 0 . Let $I^{\zeta}$ denote the ideal in $K[z]=$ $K\left[z_{1}, \ldots, z_{r}\right]$ generated by $\{f(\zeta z) \mid f(y) \in I\}$ and put

$$
\left\{\begin{array}{l}
\mathbf{E}=\left\{\mu \mid \mu \in \mathbf{Z}_{0}^{r}, z^{\mu} \in \operatorname{in}\left(z ; I^{\zeta}\right)\right\} \\
\mathbf{E}_{i}=\left\{\mu^{\prime} \mid \mu^{\prime} \in \mathbf{Z}_{0}^{i},\left(\mu^{\prime}, 0, \ldots, 0\right) \in \mathbf{E}\right\} \quad \text { for } 0 \leq i \leq r
\end{array}\right.
$$

Theorem (A.1) ([U, Theorem 2.2]). If $\left(\mu_{1}, \ldots, \mu_{p}, \ldots, \mu_{q}, \ldots, \mu_{r}\right) \in \mathbf{E}$ with $1 \leq$ $p<q \leq r, \mu_{q}>0$, then $\left(\mu_{1}, \ldots, \mu_{p}+t, \ldots, \mu_{q}-t, \ldots, \mu_{r}\right) \in \mathbf{E}$ for all $t$ such that $\left(\begin{array}{c}\mu_{q} \\ t\end{array}\right) \not \equiv 0(\bmod \operatorname{char}(k)), 0 \leq t \leq \mu_{q}$.

Proof. See [U]. There the argument is carried out with the usual lexicographic order, but it also works well with respect to the order $\prec$ we defined in the beginning of $\S 1$. 
Let $\Gamma_{i}, \Gamma_{i}^{\prime}, \Delta_{i}(1 \leq i \leq r-1)$ be the subsets of $\mathbf{Z}_{0}^{i}$ defined inductively as follows:

$$
\begin{aligned}
& \Gamma_{i}^{\prime}=\mathbf{Z}_{0}^{i} \backslash\left(\mathbf{E}_{i} \cup \bigcup_{j=1}^{i-1} \Gamma_{j} \times \mathbf{Z}_{0}^{i-j}\right), \\
& \Delta_{i}=\left\{\delta \mid \delta \in \Gamma_{i}^{\prime} \text { and }(\delta, w) \in \mathbf{E}_{i+1} \text { for some } w>0\right\}, \\
& \Gamma_{i}=\Gamma_{i}^{\prime} \backslash \Delta_{i} .
\end{aligned}
$$

We put $\Delta_{0}=\{\varnothing\}$ for convenience sake and further define

$$
\Delta_{r}=\varnothing, \quad \Gamma_{r}=\mathbf{Z}_{0}^{r} \backslash\left(\mathbf{E} \cup \bigcup_{j=1}^{r-1} \Gamma_{j} \times \mathbf{Z}_{0}^{r-j}\right)
$$

Since $\left(\mu_{1}, \ldots, \mu_{i}, \sum_{j=i+1}^{r} \mu_{j}, 0, \ldots, 0\right) \in \mathbf{E}$ for all $\left(\mu_{1}, \ldots, \mu_{r}\right) \in \mathbf{E}, i \quad(0 \leq i \leq$ $r)$ by (A.1), the intersection $\mathbf{E}_{i} \cap\left(\Gamma_{j} \times \mathbf{Z}_{0}^{i-j}\right)$ is empty for $1 \leq j \leq i \leq r$. Put $w(\delta)=\min \left\{w \mid(\delta, w) \in \mathbf{E}_{i+1}\right\}>0, \mu(i, \delta)=(\delta, w(\delta), 0, \ldots, 0) \in \mathbf{Z}_{0}^{r}$ for each pair $i, \delta \quad\left(0 \leq i \leq r-1, \delta \in \Delta_{i}\right)$, where $\mu(0, \varnothing)=(w(\varnothing), 0, \ldots, 0)$ for $i=0$. By the definition of $\Gamma_{i}, \Delta_{i}$ we have

$$
\begin{aligned}
\Delta_{i} \cup \Gamma_{i} & =\bigcup_{\delta \in \Delta_{i-1}} \delta \times[0, w(\delta)) \text { for } 1 \leq i \leq r, \\
\mathbf{E}_{i} \backslash\left(\mathbf{E}_{i-1} \times \mathbf{Z}_{0}\right) & =\bigcup_{\delta \in \Delta_{i-1}} \delta \times[w(\delta), \infty) \text { for } 1 \leq i \leq r, \\
\mathbf{E} & =\bigcup_{i=1}^{r} \bigcup_{\delta \in \Delta_{i-1}}\left(\mu(i-1, \delta)+\mathbf{Z}_{0}(i-1)\right), \\
\mathbf{Z}_{0}^{r} & =\mathbf{E} \cup\left(\bigcup_{i=1}^{r} \Gamma_{i} \times \mathbf{Z}_{0}^{r-i}\right)
\end{aligned}
$$

with $\mathbf{Z}_{0}(i)=\left\{\alpha=\left(\alpha_{1}, \ldots, \alpha_{r}\right) \mid \alpha \in \mathbf{Z}_{0}^{r}, \alpha_{j}=0\right.$ for $\left.1 \leq j \leq i\right\}$. Note that the right-hand sides are all disjoint unions. As is seen by (A.2), each $\Delta_{i}$ is a finite set. Moreover if $\Delta_{i}=\varnothing$ for some $i(1 \leq i \leq r)$, then $\Gamma_{j}=\Delta_{j}=\varnothing$ for all $j(i<j \leq r)$. Put $c=\max \left\{p \mid 0 \leq p<r, \Delta_{r-p}=\varnothing\right\}, m_{i}=\sharp \Delta_{i-1} \geq 1$ $(1 \leq i \leq r-c)$ and denote the multiexponents $\mu(i-1, \delta)\left(\delta \in \Delta_{i-1}\right)$ by $\rho(i, l) \quad\left(1 \leq l \leq m_{i}\right)$ for $1 \leq i \leq r-c$. With this notation, let $F_{l}^{\prime i}$ be a homogeneous polynomial in $I^{\zeta}$ such that $\operatorname{in}\left(z ; F_{l}^{\prime i}\right)=z^{\rho(i, l)}$ for each $i, l$ $\left(1 \leq i \leq r-c, 1 \leq l \leq m_{i}\right)$ and let $N\left(z ; I^{\zeta}\right)$ denote the linear subspace of $K[z]$ spanned over $K$ by the monomials $z^{\prime \prime}\left(\nu \in \bigcup_{i=1}^{r} \Gamma_{i} \times \mathbf{Z}_{0}^{r-i}\right)$. We will apply (4.3), replacing $k$ and $x$ by $K$ and $z$ respectively. Since the right-hand sides of (A.3), (A.4) are disjoint unions, the polynomials $F_{l}^{\prime i}$ satisfy (4.3.1) and besides the vector space $N$ defined in (4.3) coincides with $N\left(z ; I^{\zeta}\right)$. There is therefore a homogeneous $F_{l}^{\prime \prime i} \in N\left(z ; I^{\zeta}\right)$ of the same degree as $F_{l}^{\prime i}$ such that 
$\left(F_{l}^{\prime i}-z^{\rho(i, l)}\right)-{F_{l}^{\prime \prime i}}^{\prime i} I^{\zeta}$ for every $i, l$ by (4.3.2). Put $F_{l}^{i}=z^{\rho(i, l)}+F_{l}^{\prime \prime i} \in I^{\zeta}$. Then $\operatorname{in}\left(z ; I^{\zeta}\right) \cap N\left(z ; I^{\zeta}\right)=0, \operatorname{in}\left(z ; F_{l}^{i}\right)=z^{\rho(i, l)}$ and the polynomials $F_{l}^{i}$ $\left(1 \leq i \leq r-c, 1 \leq l \leq m_{i}\right)$ also satisfy (4.3.1). Hence

$$
I^{\zeta}=\bigoplus_{i=1}^{r-c} \bigoplus_{l=1}^{m_{i}} F_{l}^{i} K[z(i-1)], \quad K[z]=I^{\zeta} \oplus N\left(z ; I^{\zeta}\right) .
$$

On the other hand by the construction of $I^{\zeta}$, there is a polynomial $a(\zeta) \in$ $k[\zeta] \backslash\{0\}$ such that $a(\zeta) F_{l}^{i} \in \sum_{f(y) \in I} k[\zeta]\left[z_{1}, \ldots, z_{r}\right] f(\zeta z)$ and all coefficients of $a(\zeta) F_{l}^{i}$ lie in $k[\zeta]$ for all $i, l$. Now put $n_{l}^{i}=\operatorname{deg}\left(F_{l}^{i}\right), n^{i}=\left(n_{1}^{i}, \ldots, n_{m_{i}}^{i}\right)$, $U=\left\{\left(y_{1}, \ldots, y_{r}\right) \Upsilon \mid \Upsilon \in G L(r, k), a\left({ }^{t} \Upsilon^{-1}\right) \neq 0\right\}$. Here we may assume $n^{i}$ $(1 \leq i \leq r-c)$ are nondecreasing sequences. For each $x=\left(y_{1}, \ldots, y_{r}\right) \Upsilon \in U$, the polynomials obtained from $F_{l}^{i}\left(1 \leq i \leq r-c, 1 \leq l \leq m_{i}\right)$ by substituting ${ }^{t} \Upsilon^{-1}$ for $\zeta$ fulfill all the requirements stated in (1.1) with respect to $x$.

\section{REFERENCES}

[A1] M. Amasaki, Preparatory structure theorem for ideals defining space curves, Publ. RIMS Kyoto Univ. 19 (1983), 493-518.

[A2] On the structure of arithmetically Buchsbaum curves in $\mathbf{P}_{k}^{3}$, Publ. RIMS Kyoto Univ. 20 (1984), 793-837.

[A3] Examples of nonsingular irreducible curves which give reducible singular points of $\operatorname{red}\left(\mathrm{H}_{d, g}\right)$, Publ. RIMS Kyoto Univ. 21 (1985), 761-786.

[A4] Curves in $\mathbf{P}^{3}$ whose ideals are simple in a certain numerical sense, Publ. RIMS Kyoto Univ. 23 (1987), 1017-1052. [A5] $\frac{}{1-8 .}$ Integral arithmetically Buchsbaum curves in $\mathbf{P}^{3}$, J. Math. Soc. Japan 41 (1989),

[AB] M. Auslander and D. Buchsbaum, Codimension and multiplicity, Ann. of Math. 68 (1958), 625-657.

[BBM] E. Ballico, G. Bolondi and R. Mirò-Roig, Numerical invariants of rank 2 arithmetically Buchsbaum sheaves, preprint, U.T.M. 222 (1987).

[BM1] G. Bolondi and J. Migliore, Classification of maximal rank curves in the liaison class $L_{n}$, Math. Ann. 277 (1987), 585-603.

[BM2] _ Buchsbaum liaison classes, preprint, (1987).

[BM3] The Lazarsfeld-Rao and Zeuthen problems for Buchsbaum curves, preprint, U.T.M. 228 (1987).

[BM4] - The structure of an even liaison class, preprint, U.T.M. 239 (1988).

[BS] D. Bayer and M. Stillman, A criterion for detecting m-regularity, Invent. Math. 87 (1987), $1-11$.

[C] M. Chang, Characterization of arithmetically Buchsbaum subschemes of codimension 2 in $\mathbf{P}^{n}$, preprint, (1988).

[EF] P. Ellia et M. Fiorentini, Quelques remarques sur les courbes arithmetiquement Buchsbaum de l'espace projectif, preprint 94, Università di Ferrara.

[EG] D. Eisenbud and S. Goto, Linear free resolutions and minimal multiplicity, J. Algebra 88 (1984), 89-133.

[Ga] A. Galligo, A propos du théorème de préparation, Fonctions de Plusieurs Variables Complexes, Lecture Notes in Math., vol. 409, Springer-Verlag, Berlin, Heidelberg, New York, 1974, pp. 543-579. 
[GM1] A. V. Geramita and J. Migliore, On the ideal of an arithmetically Buchsbaum curve, J. Pure Appl. Algebra 54 (1988), 215-247.

[GM2] Generators for the ideal of an arithmetically Buchsbaum curve, preprint, Queen's University 17 (1987).

[Go1] S. Goto, Maximal Buchsbaum modules over regular local rings, Proc. 7th Sympos. Commutative Algebra, Kyoto 1985, pp. 82-89.

[Go2] - Maximal Buchsbaum modules over regular local rings and a structure theorem for generalized Cohen-Macaulay modules, Commutative Algebra and Combinatorics, Advanced Studies in Pure Mathematics, Kinokuniya, Tokyo; North-Holland, Amsterdam, 1987, pp. 39-64.

[GP] L. Gruson et C. Peskine, Genre des courbes de l'espace projectif, Algebraic Geometry, Lecture Notes in Math., vol. 687, Springer-Verlag, Berlin, Heidelberg, New York, 1978, pp. 31-59.

[Gr] H. Grauert, Über die Deformation isolierter Singularitäten analytischer Mengen, Invent. Math. 15 (1972), 171-198.

[H] H. Hironaka, Bimeromorphic smoothing of complex analytic spaces, Notes, Warwick University, 1971.

[HU] H. Hironaka and T. Urabe, Introduction to analytic spaces, Asakura, Tokyo, 1982. (Japanese)

[M] H. Matsumura, Commutative algebra, Benjamin, New York, 1970.

[MM] F. Mora and H. M. Möller, New constructive methods in classical ideal theory, J. Algebra 100 (1986), 138-178.

[SV] J. Stückrad and W. Vogel, Buchsbaum rings and applications, Springer-Verlag, Berlin, Heidelberg, New York, 1986.

[U] T. Urabe, On Hironaka's monoideal, Publ. RIMS Kyoto Univ. 15 (1979), 279-287.

Research Institute for Mathematical Sciences, Kyoto University, Kyoto 606, Japan 